\title{
HIGH FREQUENCY PHOTOACOUSTIC DETECTION OF RED BLOOD CELL AGGREGATION
}

by

Fayruz Kibria

B.Sc. Hons., York University, 2012

\author{
A thesis \\ presented to Ryerson University \\ in partial fulfillment of the \\ requirements for the degree of \\ Master of Science \\ in the Program of \\ Biomedical Physics
}

Toronto, Ontario, Canada, 2016

(C) Fayruz Kibria 2016 


\section{AUTHOR'S DECLARATION}

I hereby declare that I am the sole author of this thesis. This is a true copy of the thesis, including any required final revisions, as accepted by my examiners.

I authorize Ryerson University to lend this thesis to other institutions or individuals for the purpose of scholarly research.

I further authorize Ryerson University to reproduce this thesis by photocopying or by other means, in total or in part, at the request of other institutions or individuals for the purpose of scholarly research.

I understand that my thesis may be made electronically available to the public.

Fayruz Kibria 


\title{
Abstract
}

\section{High Frequency Photoacoustic Detection of Red Blood Cell Aggregation}

\author{
Fayruz Kibria \\ M. Sc. Biomedical Physics \\ Ryerson University, Toronto, 2016
}

Red blood cell (RBC) aggregation was correctly reported in early 1768 as the increased deposition for blood to form spots of red in pathological conditions. However only recently have there been advances in technology to allow possible detection of RBC aggregation in vivo. Photoacoustic imaging (PA) shows a promising future in the detection of simultaneous in vivo $\mathrm{RBC}$ aggregation and oxygen saturation.

This work presents, for the first time, the results of investigating aggregated RBCs using high frequency (> $20 \mathrm{MHz}$ ) photoacoustic (PA) imaging. Aggregation was induced by using 3\% w/v concentration of $70 \mathrm{kDa}$ Dextran and PA measurements were taken by a $25 \mathrm{MHz}$ center frequency transducer. It was found that the spectral slope (SS) of the photoacoustic signals decreased by $\sim 0.25 \mathrm{~dB} / \mathrm{MHz}$ with $\mathrm{RBC}$ aggregation. The results are consistent with the findings of low frequency PA RBC aggregation study which also reported decrease in SS with increased aggregation. 


\section{Acknowledgements}

My supervisor Dr. Michael Kolios has been a great mentor. His enthusiasm, passion and thirst for knowledge are unparalleled. I found his patience, guidance and stimulating pedagogy invaluable during this research. He is a true leader who builds and directs an inquisitive research team. I am deeply grateful to him for granting me this opportunity to pursue work in a greatly rewarding field.

I am very thankful to my supervisory committee members Dr. Raffi Karshafian, Dr. Carl Kumaradas and Dr. Alexandre Douplik who have with their scholarly criticism and advice helped me grow as a researcher.

Members of the Kolios group, especially Muhanad Fadhel, Eno Hysi, Dr. Eric Strohm, Dr. Lauren Wirtzfeld and Elizabeth Berndl are acknowledged and thanked for taking the time to provide scientific and professional feedback.

Lots of love to my sweet daughter Hazel Huq, my anchor. She has been very patient, loving and understanding. I thank her for being my personal cheerleader.

No amount of gratitude can repay my parents Dr. G.M. Kibria and Dr. Nargis Akhter. Looking back in time when they were completing graduate degrees while raising three children I am in awe. I am here and able only because they gave me unconditional love and support. 
To my parents who sacrificed a lot to give their children the best possible education. Broke all gender stereotypes for their daughters and made the sky the limit. Sheltered me in storms and set me free to search for happiness. I hope I have made you proud. 


\section{Table of contents}

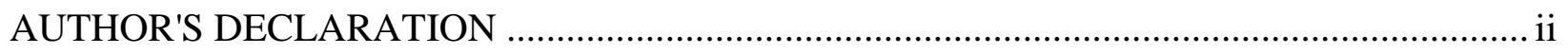

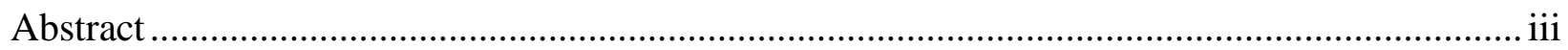

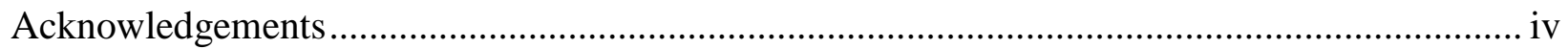

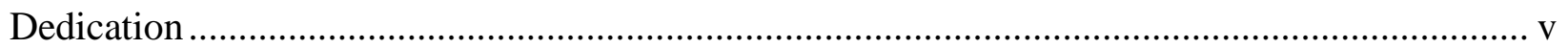

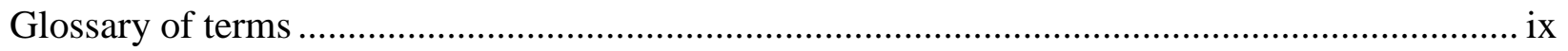

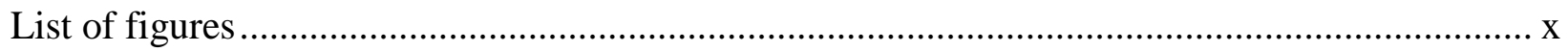

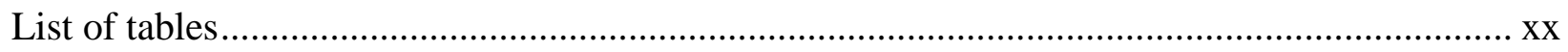

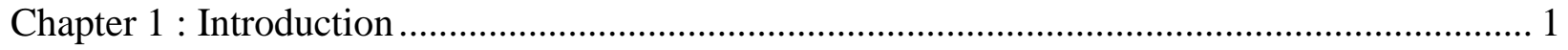

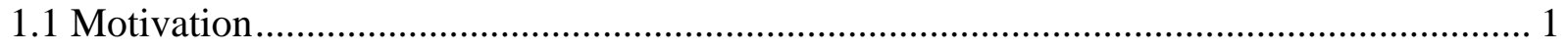

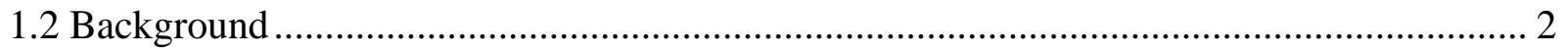

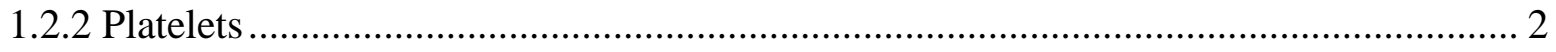

1.2.3 White blood cells ....................................................................................... 2

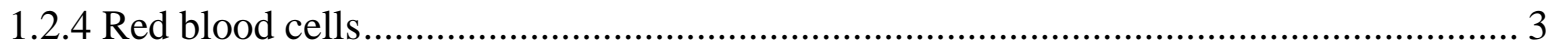

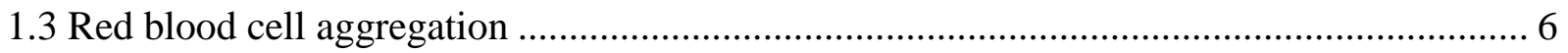

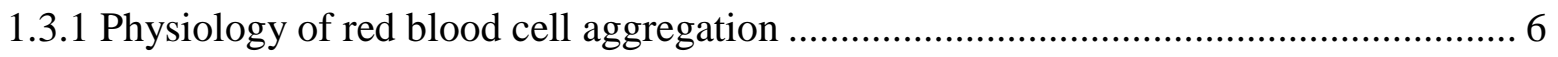

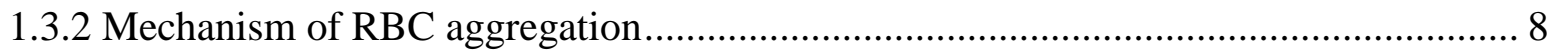

1.3.3 Factors affecting red blood cell aggregation............................................................ 9

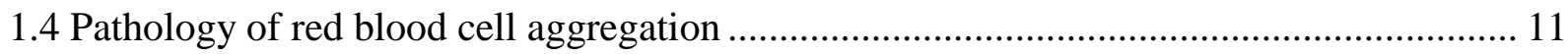

1.5 Current red blood cell aggregation quantification techniques .......................................... 13

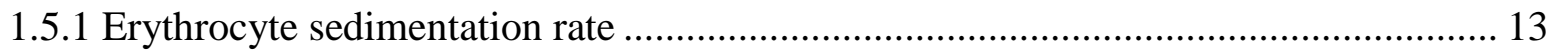

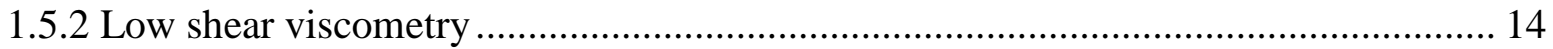

1.5.3 Microscopic observations and image analysis .......................................................... 14

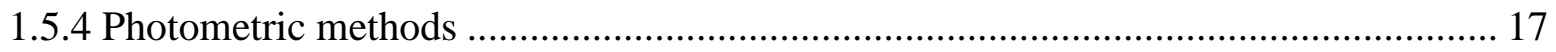

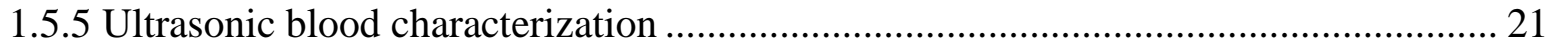

1.6 Current biomedical applications of photoacoustics ........................................................ 24

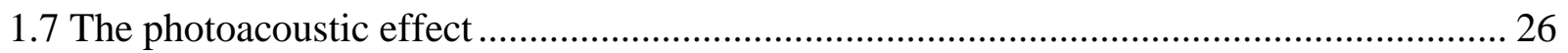

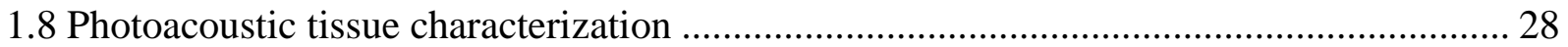

1.8.1 Using photoacoustics to assess RBC aggregation ................................................... 29

1.8.2 Using photoacoustics to assess RBC oxygen saturation.......................................... 32

1.8.3 Using photoacoustics to assess blood sedimentation................................................. 33

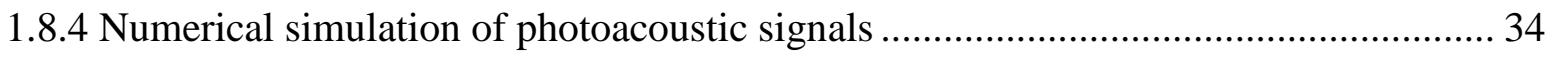

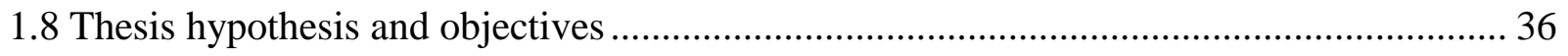




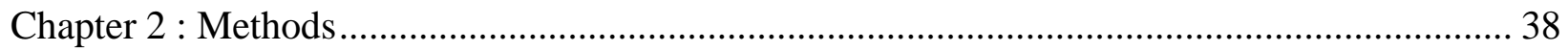

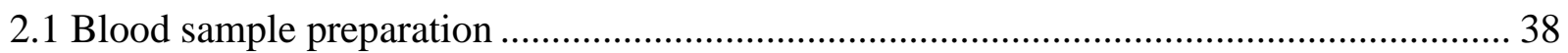

2.2 Hemocytometer image collection and blood aggregation assessment................................ 40

2.2.1 Sample loading and microscopic image acquisition.................................................. 40

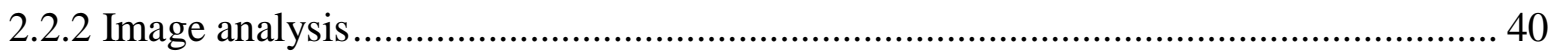

2.3 Photoacoustic data acquisition and analysis .................................................................. 42

2.3.1 Blood vessel mimicking phantom preparation ....................................................... 42

2.3.2 VEVO 2100 / LAZR Photoacoustic imaging system ................................................ 44

2.3.3 Blood phantom data acquisition....................................................................................... 45

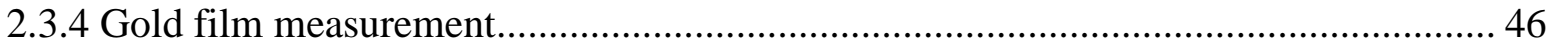

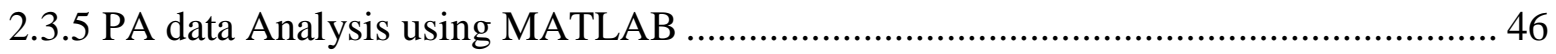

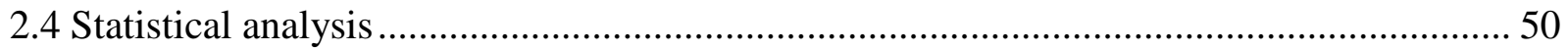

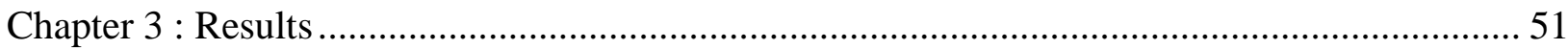

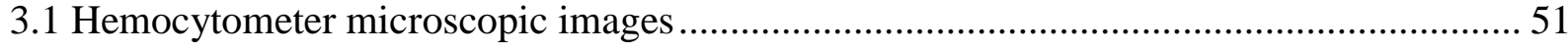

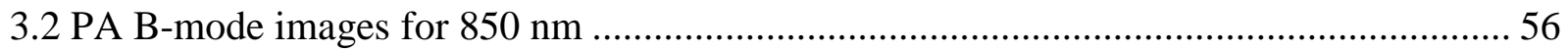

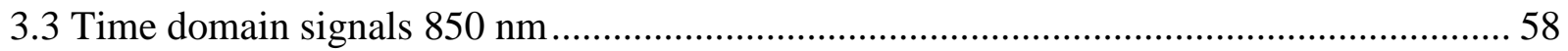

3.4 Signals obtained from the proximal edge of the vessels at $850 \mathrm{~nm}$................................... 59

3.4.1 Time domain signals ........................................................................................ 59

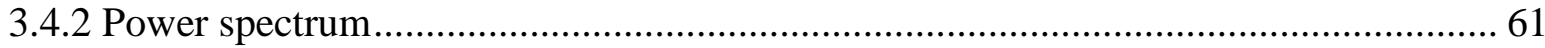

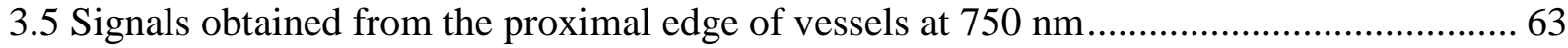

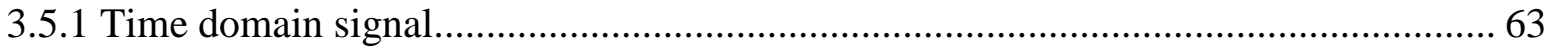

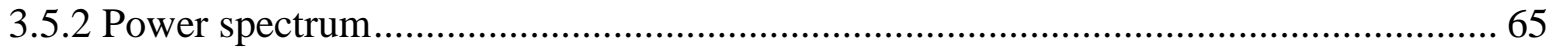

3.6 Oxygen saturation determination from the proximal edge signals ........................................ 67

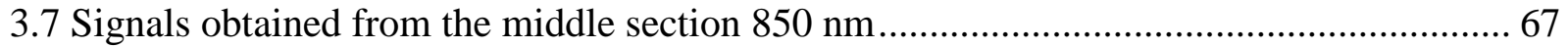

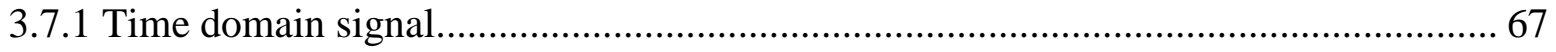

3.7.2 Power spectrum ............................................................................................... 71

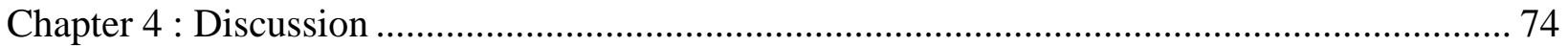

4.1 Microscopic aggregation quantification ……………................................................... 74

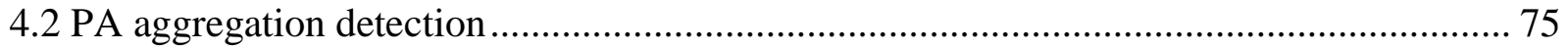

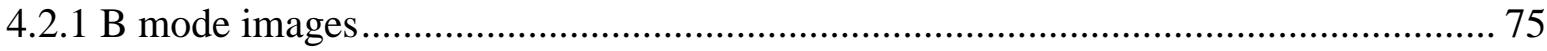

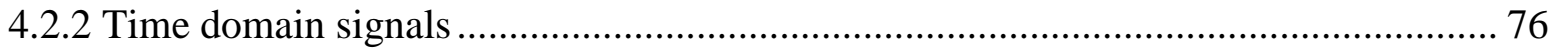

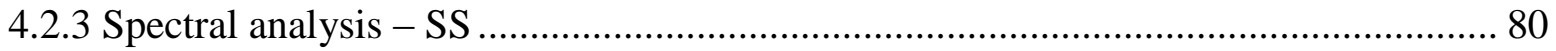

4.2.3 Spectral analysis - MBF ................................................................................ 81

4.3 Photoacoustic oxygen saturation for aggregated samples .................................................. 82 


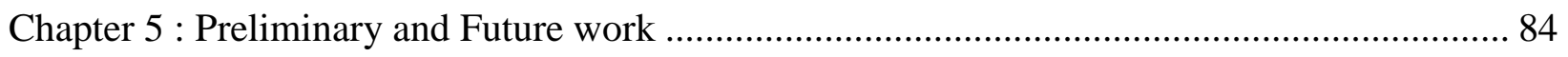

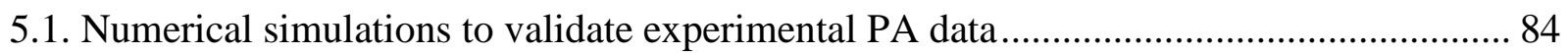

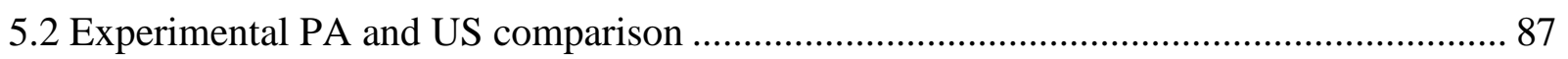

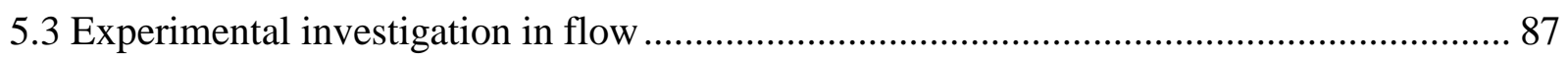

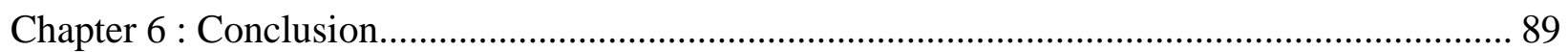

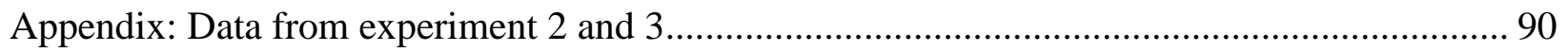

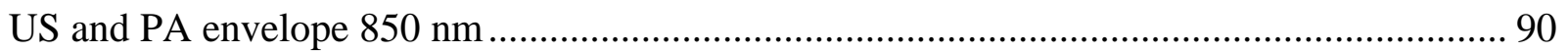

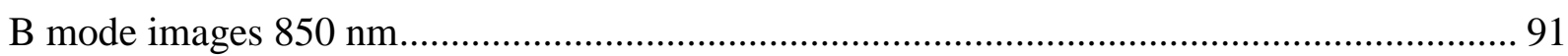

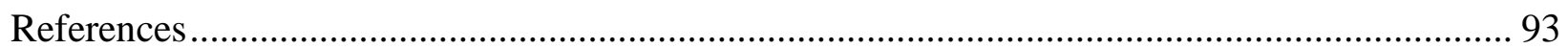




\section{Glossary of terms}

\begin{tabular}{|c|c|c|c|}
\hline $\mathrm{AG}$ & Aggregated & $\mathrm{RBC}$ & Red blood cell \\
\hline ATP & Adenosine triphosphate & $\mathrm{RF}$ & Radio frequency \\
\hline BSC & Backscattering coefficient & ROI & Region of interest \\
\hline CAM & Chorioallantoic membrane & SA & Signal amplitude \\
\hline DEX & Dextran & SFSE & Structure factor size estimator \\
\hline DMEM & Dulbecco's modified essential medium & SNR & Signal to noise ratio \\
\hline ESR & Erythrocyte sedimentation rate & $\mathrm{SO}_{2}$ & Oxygen saturation \\
\hline FBS & Fetal bovine serum & SS & Spectral slope \\
\hline FFT & Fast Fourier transform & US & Ultrasound \\
\hline $\mathrm{Hb}$ & Deoxyhemoglobin & & \\
\hline $\mathrm{HbO}_{2}$ & Oxyhemoglobin & & \\
\hline $\mathrm{HCT}$ & Hematocrit & & \\
\hline IQ & In-phase and quadrature out of phase & & \\
\hline LPS & Lipopolysachcharide & & \\
\hline $\mathrm{MBF}$ & Midband fit & & \\
\hline NA & Non aggregated & & \\
\hline NO & Nitric oxide & & \\
\hline OCT & Optical coherence tomography & & \\
\hline PA & Photoacoustic & & \\
\hline PBS & Phosphate buffered saline & & \\
\hline $\mathrm{PO}_{2}$ & Partial pressure of oxygen & & \\
\hline QUS & Quantitative ultrasound & & \\
\hline
\end{tabular}




\section{List of figures}

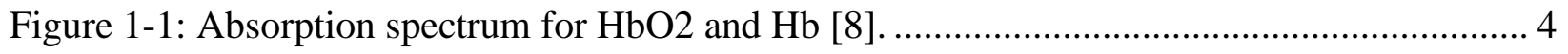

Figure 1-2: AG human blood, showing formation of rouleaux and clusters. ............................ 6

Figure 1-3: Relation between DEX-70 at different concentrations and amount of aggregation

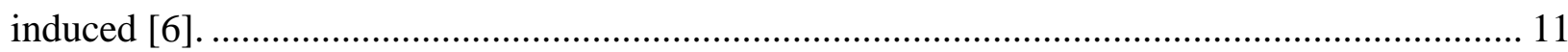

Figure 1-4: RBCs were suspended in autologous plasma to induce aggregation. (a) Shows RBC rouleaux formation in suspension, (b) uniform spherical clusters forming and (c) packed clusters

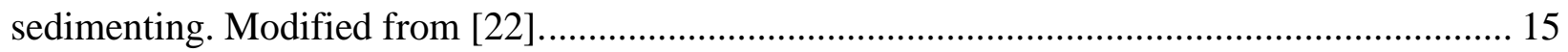

Figure 1-5: Human blood suspended in 10\% HCT with 0.5\% DEX-500, under different shearing

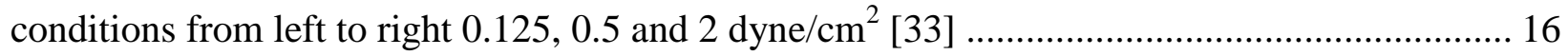

Figure 1-6: The intensity of light transmitted through the sample of RBC suspension in $4 \% \mathrm{w} / \mathrm{v}$

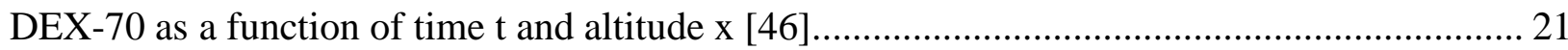

Figure 1-7: B mode images showing blood echogenicity from AG whole porcine blood (left) and NA porcine RBC (right) [47]. 21

Figure 1-8: (Left) power spectrum obtained from tissue (solid) and a glass plate as a planar US reflector (dashed). (Right) tissue power spectrum after normalization [49]............................. 22 Figure 1-9: Theoretical PA signatures for spherical droplets with density and sound speed

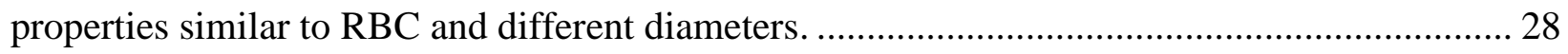
Figure 1-10: Sample representative of realizations of (a) NA and (c) AG RBCs radius of gyration 12.24 $\mu \mathrm{m}$ simulated, (b) shows the change in monotonous rise in PA power spectra amplitude with increasing HCT for NA RBCs and (d) shows the changes in peak positions and amplitudes in PA power spectrum as a function of different levels of aggregation [1] ............................. 30 Figure 1-11: (a) PA SS for $750 \mathrm{~nm}$ wavelength, (b) PA SS for $1064 \mathrm{~nm}$ wavelength, (c) PA MBF for $750 \mathrm{~nm}$ wavelength, (b) PA MBF for $1064 \mathrm{~nm}$ wavelength. Error bars denote standard

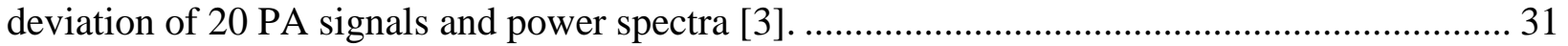
Figure 1-12: Experimentally measured PA SO2 for different levels of aggregation and HCTs of 10, 20 and 40\%. DEX-70 concentration of 3\% was most aggregating and DEX-70 0\% was non aggregating DEX-70 of concentration $1 \%$ and $8 \%$ were moderately aggregating. Results were obtained using samples of washed porcine RBCs [3]. 33 
Figure 1-13: Estimation of sedimentation in 5 mins using PA and OCT measurement data points

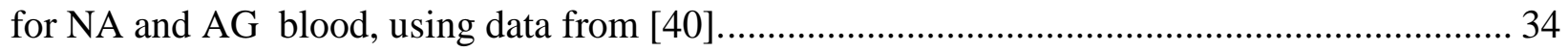

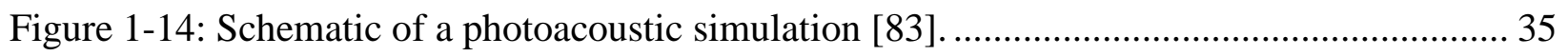

Figure 1-15: (Left) Energy deposition profile for an spherical absorber with the positions of 3 detectors specified, (right) corresponding PA A line signals from the three detectors [83]......... 36 Figure 2-1: Agar phantom setup with blood sample inside and prepared for imaging with the LZ

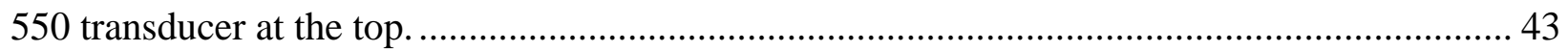

Figure 2-2: Schematic of the phantom setup for PA measurements........................................... 43 Figure 2-3: The Vevo LAZR Photoacoustic imaging system. The four main components of the imaging system are the Vevo LAZRTight, LZ series transducer, Vevo LAZR cart and the Vevo

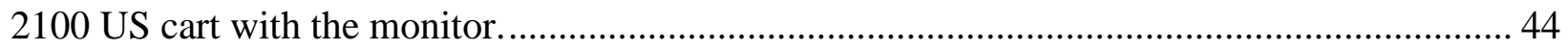

Figure 2-4: Typical PA gold film frequency response for LZ-550 transducer.............................. 46 Figure 3-1: Microscopic images of (left) NA washed RBCs in 0\% DEX-70 (right) AG washed

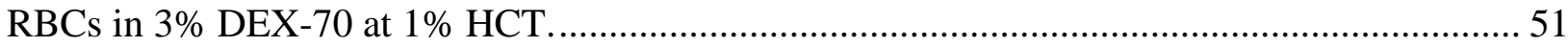

Figure 3-2: Thresholded segmented images after particle analysis (left) NA sample, and (right) AG sample. 52

Figure 3-3: Size distribution in $\mu \mathrm{m}^{2}$ for RBC units in the 0\% DEX-70 sample (NA) and for washed RBC units in 3\% DEX-70 solution (AG) . Each unit is $n$ number of RBCs attached together, where $\mathrm{n}$ was seen to vary experimentally from 1 to 13 . 53 Figure 3-4: Zoomed in image of an AG sample. The arrows point to the units selected for projected $\mathrm{RBC}$ side area calculation. 53 Figure 3-5: (Top) axial intensity profile for typical single RBC on its side and (bottom) axial intensity profile for a typical rouleaux. The width of single cell is about $2 \mu \mathrm{m}$ and the width of the rouleaux is about $6 \mu \mathrm{m}$. For the rouleau the dips indicate aggregation of four RBCs in this unit.

Figure 3-6: Percentage of RBCs in units with incremental number of RBCs per unit. The results for NA sample show about $90 \%$ of the cells are single cells. Most of the units in the AG samples contained four cells. 55

Figure 3-7: Co-reregistered US and PA images as seen on VEVO 2100 screen, on the left greyscale images represent US B mode images and on the right the blue and black images 
represent PA B mode images. HCTs of 10, 20 and 40\% suspended in 0\% DEX-70 and 3\% DEX70 are shown (white arrows indicate the proximal and distal edges of the vessels).................. 56 Figure 3-8: Reconstructed US B mode image (left) and PA B mode image (right). B mode image from experiment 1 is shown here. The intensity values are measured in $\mathrm{dB}$. 57 Figure 3-9: PA ROI with vessel realigned (left) and noise from a region between the transducer and phantom (right). B mode image from experiment 1 is shown here. The intensity values are measured in $\mathrm{dB}$. 57

Figure 3-10: 20 A lines averaged over 20 frames were chosen per HCT per DEX-70 concentration for data analysis. (Left) unaligned A lines and (right) aligned A lines. 58 Figure 3-11: Envelopes of US (black) and PA (blue) A lines. US A lines were used to determine the beginning, middle and end of the vessels. Data from experiment 1 is shown in this figure... 58 Figure 3-12: (Inset) a zoomed image to show the start (red arrow) of the vessel as indicated by the US envelope, due to settling there is no corresponding PA signal at the vessel start. The sectioned A lines obtained by using the US measurements are shown in the bottom row. 59 Figure 3-13: Representative A line signals from the proximal edge of the vessels for $40 \%$ HCT obtained at $850 \mathrm{~nm}$ illumination. Left column is from NA samples and right column from AG samples. Exp1, exp 2 and exp 3 represent results from experiment 1, 2 and 3 respectively....... 60 Figure 3-14: Experimental SA at $40 \% \mathrm{HCT}$ for $850 \mathrm{~nm}$ illumination from the proximal edge of the vessels. SA was calculated by obtaining the peak to peak value of the signals. Error bars represent standard deviation of 20 PA RF lines. E1, E2 and E3 mean experiment 1, experiment 2 and experiment 3 respectively. 61 Figure 3-15: Power spectra and linear fit at $40 \%$ HCT for $850 \mathrm{~nm}$ illumination. The left column represents NA samples and the right column represents AG samples. Blue lines are the power spectra and red lines represent the linear fit. In each subplot there are 20 power spectra which were transformed from A lines obtained from 20 different lateral positions in the phantom. ..... 62 Figure 3-16: Experimental results for SS at $40 \%$ HCT for $850 \mathrm{~nm}$ illumination from the proximal edge of the vessel. The error bars represent standard deviation of 20 PA spectra. ....................62 62 Figure 3-17: Experimental results for $\mathrm{MBF}$ at $40 \% \mathrm{HCT}$ for $850 \mathrm{~nm}$ illumination from the proximal edge of the vessel. The error bars represent standard deviation of 20 PA spectra. 
Figure 3-18: Representative A line signals from the proximal edge of the vessels for $40 \%$ HCT obtained at $750 \mathrm{~nm}$ illumination. Left column is from NA samples and right column from AG samples

Figure 3-19: Experimental SA at $40 \% \mathrm{HCT}$ for $750 \mathrm{~nm}$ illumination. SA was calculated by obtaining the peak to peak value of the signals. Error bars represent standard deviation of 20 PA RF lines.

Figure 3-20: Power spectra and linear fit at 40\% HCT for $750 \mathrm{~nm}$ illumination. The left column represents NA samples and the right column represents AG samples. Blue lines are the power spectra and red lines represent the linear fit. In each subplot there are 20 power spectra which were transformed from A lines obtained from 20 different lateral positions in the phantom. .... 65 Figure 3-21: Experimental results for SS at $40 \% \mathrm{HCT}$ for $750 \mathrm{~nm}$ illumination from the proximal edge of the vessel. The error bars represent standard deviation of $20 \mathrm{PA}$ spectra 66 Figure 3-22: Experimental results for $\mathrm{MBF}$ at $40 \% \mathrm{HCT}$ for $750 \mathrm{~nm}$ illumination, from the signal at the proximal edge of the vessels. The error bars represent standard deviation of 20 PA spectra.

Figure 3-23: $\mathrm{SO}_{2}$ for $\mathrm{AG}$ samples for the three experiments. $\mathrm{SO}_{2}$ was calculated by using the $\mathrm{SA}$ from the proximal edge of the vessels for the $40 \%$ HCT samples.

Figure 3-24: Representative A line signals from the middle section of the vessels (top) $40 \%$ HCT, (middle) $20 \% \mathrm{HCT}$ and (bottom) 10\% HCT for $850 \mathrm{~nm}$ illumination. Left column is from NA samples and right column from AG samples. 68

Figure 3-25: Experimental SA (top) 40\% HCT, (middle) 20\% HCT and (bottom) 10\% HCT for $850 \mathrm{~nm}$ illumination. SA was calculated by obtaining the peak to peak value of the signals.. Error bars represent standard deviation of 20 PA RF lines. 70 Figure 3-26: Power spectra and linear fit (top) 40\% HCT, (middle) 20\% HCT and (bottom) 10\% HCT for $850 \mathrm{~nm}$ illumination. Left column represents NA samples and right column represents AG samples. Blue lines are the power spectra and red lines represent the linear fit. In each subplot there are 20 power spectra which were transformed from A lines obtained from 20 different lateral positions in the phantom. 72 Figure 3-27: Experimental results for MBF (top) 40\% HCT, (middle) 20\% HCT and (bottom) $10 \%$ HCT for $850 \mathrm{~nm}$ illumination. The error bars represent standard deviation of 20 PA spectra. 0\% DEX-70 represents NA samples and 3\% DEX-70 represents AG samples. The square and 
triangle represent data that suggest fast sedimentation as commented on in the discussion section.

Figure 4-1: Suggested schematic of the distribution of the NA and AG RBCs in the phantom vessels. 75

Figure 5-1: Simulated vessel geometries. (Top) 40\% HCT blood in a $1 \mathrm{~mm}$ diameter vessel with aggregates uniformly distributed. (Bottom) 40\% HCT blood with the aggregates sedimented at the distal edge. .84 Figure 5-2: (Left) simulated fluence for sample with no sedimentation, (right) simulated fluence for sample with sedimentation. 85

Figure 5-3: Energy deposition map for (left) sample simulated with no sedimentation and (right) sample with sedimentation 85 Figure 5-4: Simulated Alines for the middle element (left) uniform aggregate distribution, (right) sedimented RBC aggregates. 86 Figure 5-5: US (left) and PA (right) image from the VEVO 2100 LAZR screen showing blood flow in vivo. Obtained noninvasively from a CAM model. The PA contrast shows the phenomenon of axial migration. 88 Figure A-0-1: Envelopes of US (black) and PA (blue) A lines. US A lines were used to determine the beginning, middle and end of the vessels. Data from (top) experiment 2 (bottom) experiment 3 .......

Figure A-0-2: PA ROI with vessel realigned (left) and (right) noise from a region between the transducer and phantom. Data shown from experiment 2 is shown here. 91 Figure A-0-3: Reconstructed US B mode image. Data from experiment 2 is shown here. 91 Figure A-0-4: PA ROI with vessel realigned (left) and (right) noise from a region between the transducer and phantom. Data from experiment 3 is shown here. 92 Figure A-0-5: Reconstructed US B mode image. Data from experiment 3 is shown here. 92 


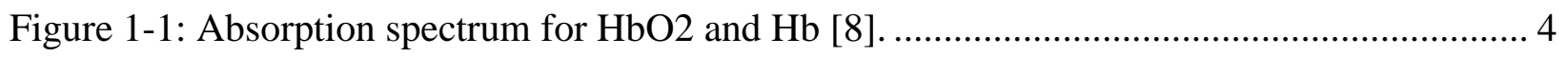

Figure 1-2: AG human blood, showing formation of rouleaux and clusters. ............................ 6

Figure 1-3: Relation between DEX-70 at different concentrations and amount of aggregation

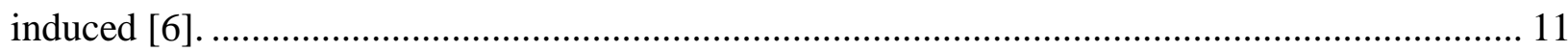

Figure 1-4: RBCs were suspended in autologous plasma to induce aggregation. (a) Shows RBC rouleaux formation in suspension, (b) uniform spherical clusters forming and (c) packed clusters

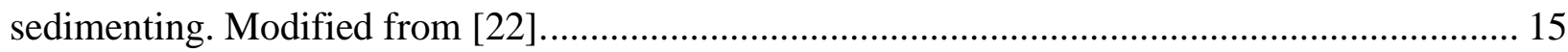

Figure 1-5: Human blood suspended in 10\% HCT with 0.5\% DEX-500, under different shearing

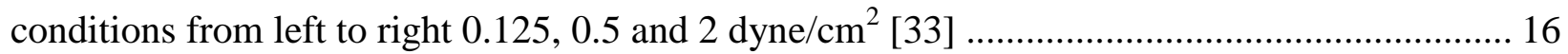

Figure 1-6: The intensity of light transmitted through the sample of RBC suspension in $4 \% \mathrm{w} / \mathrm{v}$

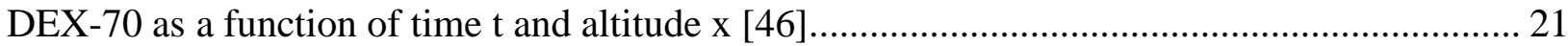
Figure 1-7: B mode images showing blood echogenicity from AG whole porcine blood (left) and NA porcine RBC (right) [47]. 21

Figure 1-8: (Left) power spectrum obtained from tissue (solid) and a glass plate as a planar US reflector (dashed). (Right) tissue power spectrum after normalization [49]............................ 22 Figure 1-9: Theoretical PA signatures for spherical droplets with density and sound speed

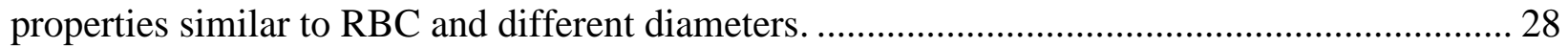
Figure 1-10: Sample representative of realizations of (a) NA and (c) AG RBCs radius of gyration $12.24 \mu \mathrm{m}$ simulated, (b) shows the change in monotonous rise in PA power spectra amplitude with increasing HCT for NA RBCs and (d) shows the changes in peak positions and amplitudes in PA power spectrum as a function of different levels of aggregation [1] ............................. 30 Figure 1-11: (a) PA SS for $750 \mathrm{~nm}$ wavelength, (b) PA SS for $1064 \mathrm{~nm}$ wavelength, (c) PA MBF for $750 \mathrm{~nm}$ wavelength, (b) PA MBF for $1064 \mathrm{~nm}$ wavelength. Error bars denote standard

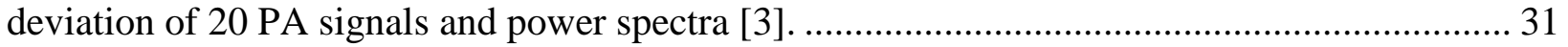
Figure 1-12: Experimentally measured PA SO2 for different levels of aggregation and HCTs of 10, 20 and 40\%. DEX-70 concentration of 3\% was most aggregating and DEX-70 0\% was non aggregating DEX-70 of concentration $1 \%$ and $8 \%$ were moderately aggregating. Results were obtained using samples of washed porcine RBCs [3] ...................................................... 33

Figure 1-13: Estimation of sedimentation in 5 mins using PA and OCT measurement data points

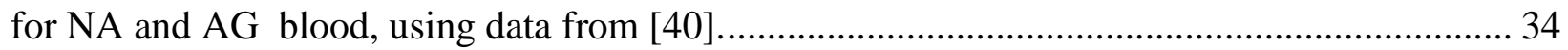
Figure 1-14: Schematic of a photoacoustic simulation [83] ............................................. 35 
Figure 1-15: (Left) Energy deposition profile for an spherical absorber with the positions of 3 detectors specified, (right) corresponding PA A line signals from the three detectors [83]........ 36 Figure 2-1: Agar phantom setup with blood sample inside and prepared for imaging with the LZ

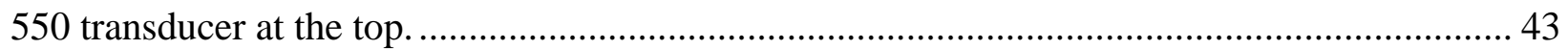

Figure 2-2: Schematic of the phantom setup for PA measurements..................................... 43 Figure 2-3: The Vevo LAZR Photoacoustic imaging system. The four main components of the imaging system are the Vevo LAZRTight, LZ series transducer, Vevo LAZR cart and the Vevo

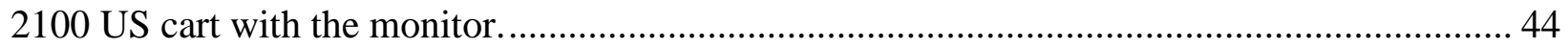

Figure 2-4: Typical PA gold film frequency response for LZ-550 transducer......................... 46 Figure 3-1: Microscopic images of (left) NA washed RBCs in 0\% DEX-70 (right) AG washed RBCs in $3 \%$ DEX-70 at $1 \%$ HCT. 51 Figure 3-2: Thresholded segmented images after particle analysis (left) NA sample, and (right) AG sample. 52

Figure 3-3: Size distribution in $\mu \mathrm{m}^{2}$ for RBC units in the $0 \%$ DEX-70 sample (NA) and for washed RBC units in 3\% DEX-70 solution (AG) . Each unit is n number of RBCs attached together, where $\mathrm{n}$ was seen to vary experimentally from 1 to 13 . 53 Figure 3-4: Zoomed in image of an AG sample. The arrows point to the units selected for projected $\mathrm{RBC}$ side area calculation 53 Figure 3-5: (Top) axial intensity profile for typical single RBC on its side and (bottom) axial intensity profile for a typical rouleaux. The width of single cell is about $2 \mu \mathrm{m}$ and the width of the rouleaux is about $6 \mu \mathrm{m}$. For the rouleau the dips indicate aggregation of four RBCs in this unit.

Figure 3-6: Percentage of RBCs in units with incremental number of RBCs per unit. The results for NA sample show about $90 \%$ of the cells are single cells. Most of the units in the AG samples contained four cells. 55

Figure 3-7: Co-reregistered US and PA images as seen on VEVO 2100 screen, on the left greyscale images represent US B mode images and on the right the blue and black images represent PA B mode images. HCTs of 10, 20 and 40\% suspended in 0\% DEX-70 and 3\% DEX70 are shown (white arrows indicate the proximal and distal edges of the vessels)..... 56 Figure 3-8: Reconstructed US B mode image (left) and PA B mode image (right). B mode image from experiment 1 is shown here. The intensity values are measured in $\mathrm{dB}$. 57 
Figure 3-9: PA ROI with vessel realigned (left) and noise from a region between the transducer and phantom (right). B mode image from experiment 1 is shown here. The intensity values are measured in $\mathrm{dB}$.

Figure 3-10: 20 A lines averaged over 20 frames were chosen per HCT per DEX-70 concentration for data analysis. (Left) unaligned A lines and (right) aligned A lines. 58 Figure 3-11: Envelopes of US (black) and PA (blue) A lines. US A lines were used to determine the beginning, middle and end of the vessels. Data from experiment 1 is shown in this figure... 58 Figure 3-12: (Inset) a zoomed image to show the start (red arrow) of the vessel as indicated by the US envelope, due to settling there is no corresponding PA signal at the vessel start. The sectioned A lines obtained by using the US measurements are shown in the bottom row..... 59 Figure 3-13: Representative A line signals from the proximal edge of the vessels for $40 \%$ HCT obtained at $850 \mathrm{~nm}$ illumination. Left column is from NA samples and right column from AG samples. Exp1, exp 2 and exp 3 represent results from experiment 1, 2 and 3 respectively.... 60 Figure 3-14: Experimental SA at $40 \% \mathrm{HCT}$ for $850 \mathrm{~nm}$ illumination from the proximal edge of the vessels. SA was calculated by obtaining the peak to peak value of the signals. Error bars represent standard deviation of 20 PA RF lines. E1, E2 and E3 mean experiment 1, experiment 2 and experiment 3 respectively.

Figure 3-15: Power spectra and linear fit at $40 \%$ HCT for $850 \mathrm{~nm}$ illumination. The left column represents NA samples and the right column represents AG samples. Blue lines are the power spectra and red lines represent the linear fit. In each subplot there are 20 power spectra which were transformed from A lines obtained from 20 different lateral positions in the phantom. .... 62 Figure 3-16: Experimental results for SS at $40 \%$ HCT for $850 \mathrm{~nm}$ illumination from the proximal edge of the vessel. The error bars represent standard deviation of 20 PA spectra. 62 Figure 3-17: Experimental results for $\mathrm{MBF}$ at $40 \% \mathrm{HCT}$ for $850 \mathrm{~nm}$ illumination from the proximal edge of the vessel. The error bars represent standard deviation of 20 PA spectra....... 63 Figure 3-18: Representative A line signals from the proximal edge of the vessels for $40 \%$ HCT obtained at $750 \mathrm{~nm}$ illumination. Left column is from NA samples and right column from AG samples

Figure 3-19: Experimental SA at $40 \% \mathrm{HCT}$ for $750 \mathrm{~nm}$ illumination. SA was calculated by obtaining the peak to peak value of the signals. Error bars represent standard deviation of 20 PA RF lines. 
Figure 3-20: Power spectra and linear fit at $40 \%$ HCT for $750 \mathrm{~nm}$ illumination. The left column represents NA samples and the right column represents AG samples. Blue lines are the power spectra and red lines represent the linear fit. In each subplot there are 20 power spectra which were transformed from A lines obtained from 20 different lateral positions in the phantom. ..... 65 Figure 3-21: Experimental results for SS at 40\% HCT for $750 \mathrm{~nm}$ illumination from the proximal edge of the vessel. The error bars represent standard deviation of 20 PA spectra....... 66 Figure 3-22: Experimental results for MBF at $40 \% \mathrm{HCT}$ for $750 \mathrm{~nm}$ illumination, from the signal at the proximal edge of the vessels. The error bars represent standard deviation of 20 PA spectra.

Figure 3-23: $\mathrm{SO}_{2}$ for $\mathrm{AG}$ samples for the three experiments. $\mathrm{SO}_{2}$ was calculated by using the $\mathrm{SA}$ from the proximal edge of the vessels for the $40 \%$ HCT samples.......................................... 67 Figure 3-24: Representative A line signals from the middle section of the vessels (top) $40 \%$ HCT, (middle) 20\% HCT and (bottom) 10\% HCT for $850 \mathrm{~nm}$ illumination. Left column is from

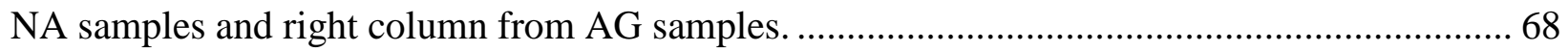
Figure 3-25: Experimental SA (top) 40\% HCT, (middle) 20\% HCT and (bottom) 10\% HCT for $850 \mathrm{~nm}$ illumination. SA was calculated by obtaining the peak to peak value of the signals.. Error bars represent standard deviation of 20 PA RF lines...................................................... 70 Figure 3-26: Power spectra and linear fit (top) 40\% HCT, (middle) 20\% HCT and (bottom) 10\% HCT for $850 \mathrm{~nm}$ illumination. Left column represents NA samples and right column represents AG samples. Blue lines are the power spectra and red lines represent the linear fit. In each subplot there are 20 power spectra which were transformed from A lines obtained from 20 different lateral positions in the phantom. 72 Figure 3-27: Experimental results for MBF (top) 40\% HCT, (middle) 20\% HCT and (bottom) $10 \%$ HCT for $850 \mathrm{~nm}$ illumination. The error bars represent standard deviation of 20 PA spectra. 0\% DEX-70 represents NA samples and 3\% DEX-70 represents AG samples. The square and triangle represent data that suggest fast sedimentation as commented on in the discussion section.

Figure 4-1: Suggested schematic of the distribution of the NA and AG RBCs in the phantom vessels. 75 
Figure 5-1: Simulated vessel geometries. (Top) 40\% HCT blood in a $1 \mathrm{~mm}$ diameter vessel with aggregates uniformly distributed. (Bottom) 40\% HCT blood with the aggregates sedimented at the distal edge.

Figure 5-2: (Left) simulated fluence for sample with no sedimentation, (right) simulated fluence for sample with sedimentation. 85

Figure 5-3: Energy deposition map for (left) sample simulated with no sedimentation and (right) sample with sedimentation. 85 Figure 5-4: Simulated Alines for the middle element (left) uniform aggregate distribution, (right) sedimented RBC aggregates. 86 Figure 5-5: US (left) and PA (right) image from the VEVO 2100 LAZR screen showing blood flow in vivo. Obtained noninvasively from a CAM model. The PA contrast shows the phenomenon of axial migration. 88 Figure A-0-1: Envelopes of US (black) and PA (blue) A lines. US A lines were used to determine the beginning, middle and end of the vessels. Data from (top) experiment 2 (bottom) experiment 3 . 90 Figure A-0-2: PA ROI with vessel realigned (left) and (right) noise from a region between the transducer and phantom. Data shown from experiment 2 is shown here ............................... 91 Figure A-0-3: Reconstructed US B mode image. Data from experiment 2 is shown here.......... 91 Figure A-0-4: PA ROI with vessel realigned (left) and (right) noise from a region between the transducer and phantom. Data from experiment 3 is shown here ......................................... 92 Figure A-0-5: Reconstructed US B mode image. Data from experiment 3 is shown here. 92 


\section{List of tables}

Table 2-1: Packed RBC, DMEM-FBS and 20\% DEX-70 in DMEM-FBS volumes added to create six samples with 40, 20, 10\% HCTs and two DEX-70 concentrations of $0 \%$ and $3 \%$...... 39 Table 2-2: Volumes (in $\mu \mathrm{L}$ ) of packed RBCs, DEX-70 plus DMEM-FBS and DMEM-FBS added to create hemocytometer samples with $1 \% \mathrm{HCT}$ and $0 \%$ and $3 \% \mathrm{DEX}-70$ concentrations........ 39

Table 2-3: Lateral distance for experiments chosen for analysis.......................................... 48 


\section{Chapter 1 : Introduction}

\subsection{Motivation}

Alterations in blood aggregation can be a valuable parameter in hematology/rheology that could bring additional prognostic information for managing widespread diseases like diabetes. Changes in blood aggregation can also be monitored for early screening and prevention of severe inflammatory reactions such as sepsis or complications during surgical procedures. The gold standard for aggregation is in vitro optical aggregometry which is not suitable for direct in vivo comparison and real-time monitoring of aggregation in patients. Theoretical work indicates that photoacoustic (PA) signals produced from the dominant optical absorbers in blood show changes that reflect the aggregation status [1]. Experimental results at low frequency have also been successfully employed to detect aggregation [2], [3]. In addition to detecting changes due to aggregation, the analysis of PA signals can also be used to quantify oxygen saturation $\left(\mathrm{SO}_{2}\right)$. The PA mean power spectrum has been shown to increase monotonically with increase in blood hematocrit (HCT) [1]. All the results indicate PA is a suitable technique that can be employed to detect $\mathrm{RBC}$ aggregation in vivo to obtain several physiological parameters of importance. Ultrasound (US) the only other noninvasive technique being investigated to quantify RBC aggregation, cannot provide the advantages related to optical generation of signals in PA. Since an elevated level of RBC aggregation is an indicator of disease, disturbance to homeostasis, or adverse effects of a clinical procedure [4], the development of real time, noninvasive techniques for aggregation detection is an ongoing field of investigation. 


\subsection{Background}

Blood constitutes of intravascular fluid in which formed elements are suspended. The fluid is called plasma and the formed elements are red blood cells (RBCs), white blood cells (WBCs) and platelets. The plasma is a straw colored liquid that carries a variety of solutes. Fibrinogen, a soluble protein produced by the liver, is an important clotting factor. Fibrinogen also plays an essential role in the formation of RBC aggregates [5]. Blood can be centrifuged to separate it into its constituent components, and the percentage volume of each component in blood can be calculated from the height of the components in the centrifuge tube. The formed elements of blood make up $45 \%$ of the blood volume and the plasma accounts for the remaining $55 \%$. HCT is the percentage volume of blood that is composed of RBCs only. In healthy humans the HCT value ranges from $36 \%$ to $53 \%$ [6].

\subsubsection{Platelets}

Platelets are small $(2-3 \mu \mathrm{m})$ colorless oval or irregularly shaped bodies. At the site of injury platelets clump together and activate plasma clotting factors which convert the soluble fibrinogen in to insoluble mesh of fibrin fibers. The blood cells are trapped in the mesh to reinforce the platelet plug. Unlike blood aggregation which is reversible, the process of blood coagulation is irreversible and the cells and components do not revert to their pre clotting state.

\subsubsection{White blood cells}

WBCs (also referred to as leukocytes) have nuclei and mitochondria and are almost invisible under the microscope unless stained. WBCs can range from 7 to $30 \mu \mathrm{m}$ in diameter and are capable of amoeboid like motion. They defend the body by several processes including 
phagocytosis, chemically mediated pathogen destruction and storing immunity information in case of future attacks. WBCs can attach to the endothelial walls of post capillary venules and squeeze between the adjacent vessel wall cells, by a process called extravasation, to reach the site of inflammation.

\subsubsection{Red blood cells}

Erythropoiesis (production of RBCs) occurs in the bone marrow by proliferation and differentiation of hematopoietic stem cells. The production rate depends on the tissue oxygen demand and the arterial blood oxygenation. The estimated production rate for the RBCs is $2 \times$ $10^{11}$ cells per day [7]. A mature $\mathrm{RBC}$ is a biconcave disk with a diameter of $7.8 \mu \mathrm{m}$ and maximum thickness near the edges of about $2 \mu \mathrm{m}$. The RBC maturation process involves production of hemoglobin in the cytoplasm and expulsion of the nucleus. Hemoglobin is the oxygen carrying molecule that gives blood its characteristic red color. Human RBCs are highly specialized to maximize oxygen transportation: the lack of nucleus ensures maximum oxygen carrying hemoglobin concentration and the unique biconcave shape facilitates increased oxygen diffusion due to greater cell surface area. RBCs are also capable of undergoing deformation in order to squeeze through the narrow capillaries and deliver oxygen to the tissue.

RBCs have a limited lifespan of 120 days in circulation after which they are phagocytosed by macrophages in the spleen and liver. The iron from the destroyed RBCs is conserved and recycled to produce new $\mathrm{RBCs}$. 


\subsubsection{Hemoglobin}

In a fully mature RBC the hemoglobin count is about 200-300 million molecules. Oxygen molecules chemically bond to hemoglobin molecules. Hemoglobin bonded with oxygen is called oxyhemoglobin $\left(\mathrm{HbO}_{2}\right)$ and one hemoglobin molecule can bond with a maximum of four oxygen molecules. When hemoglobin releases oxygen molecules it is called deoxyhemoglobin (Hb).

Each of these hemoglobin types has a unique optical absorption spectrum. This can be exploited to identify the amount of each of the hemoglobin types present in blood. Under normal circumstances the arterial blood is composed of $97 \% \mathrm{HbO}_{2}$ and the rest is varying amounts of the other forms. The following Figure 1-1 shows the absorption spectrum for $\mathrm{HbO}_{2}$ and $\mathrm{Hb}[8]$. The fact that different states of hemoglobin have distinct absorption spectra can reveal functional information about the state of blood with techniques based on optical absorbance.

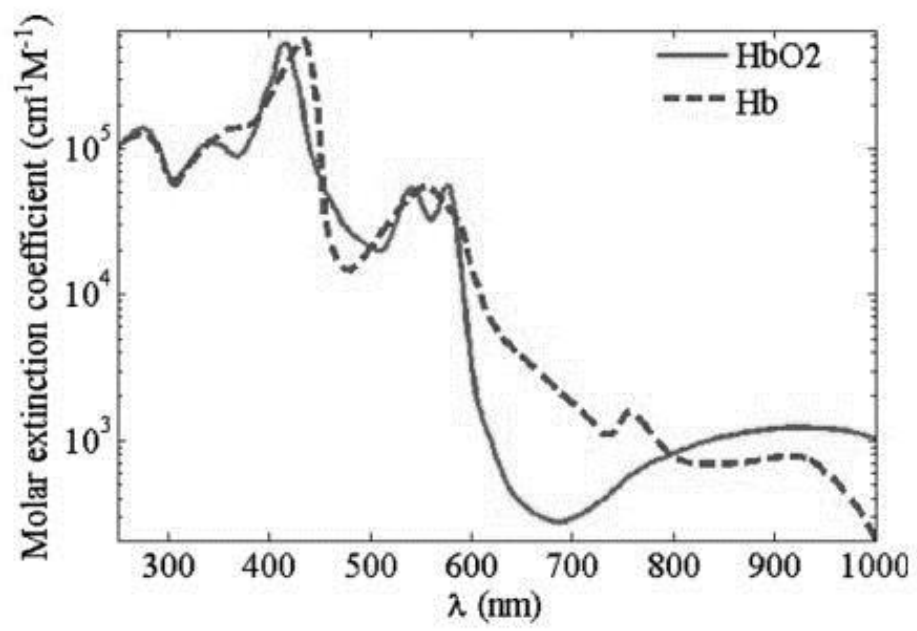

Figure 1-1: Absorption spectrum for $\mathrm{HbO} 2$ and $\mathrm{Hb}$ [8]. 


\subsubsection{Oxygen transportation}

Oxygen is important in biochemical energy generation processes due to the role it plays in electron transfer processes [9]. Partial pressure of oxygen $\left(\mathrm{PO}_{2}\right)$ is defined as the amount of oxygen physically dissolved in the blood plasma. The normal value for $\mathrm{PO}_{2}$ is $60-100 \mathrm{mmHg}$. The amount of oxygen dissolved in plasma does not contribute much directly to the delivery of oxygen to tissue. Instead, the significance of $\mathrm{PO}_{2}$ lies in the way it affects the hemoglobin $\mathrm{SO}_{2}$ in the RBCs. $\mathrm{PO}_{2}$ dictates the hemoglobin's binding affinity to oxygen. In lungs the $\mathrm{PO}_{2}$ is high and the most of the $\mathrm{Hb}$ molecules bind to oxygen $\left(\mathrm{PO}_{2}=100 \mathrm{mmHg}, \mathrm{SO}_{2}=97 \%\right)$. The lower $\mathrm{PO}_{2}$ in the systemic capillaries reduce hemoglobin oxygen affinity and cause oxygen unloading $\left(\mathrm{PO}_{2}=\right.$ $40 \mathrm{mmHg}, \mathrm{SO}_{2}=75 \%$ ). When RBCs arrive at the capillary level, several factors facilitate the release of oxygen from hemoglobin. The lower $\mathrm{PO}_{2}$ in the interstitial fluid causes net oxygen diffusion from plasma to the fluid. The lowering $\mathrm{PO}_{2}$ in plasma in turn reduces the $\mathrm{HbO}_{2}$ oxygen affinity and the net result is oxygen diffusing out from the RBC into the tissue. High carbondioxide level in tissue (a respiratory by-product) lowers local $\mathrm{pH}$, favoring oxygen unloading from $\mathrm{HbO}_{2}$. Carbon dioxide also binds to the protein chains in the hemoglobin, which causes a conformational change that facilitates oxygen release.

The role of RBC in oxygen transport is not only that of a carrier. RBCs have active mechanisms that sense tissue oxygenation via their degree of deoxygenation. RBCs have the advantage of being direct local effectors to ensure fast matching between local oxygen supply and demand [10]. Adenosine triphosphate (ATP) and nitric oxide (NO) are two bimolecular agents released from RBC in tissue hypoxia [11]. Both ATP and NO act as vasodilators increasing blood flow to tissue that has high oxygen consumption. Given the role RBCs play in oxygen transport and the 
importance of proper tissue oxygenation, it is critical under many pathological conditions to assess the level of blood oxygen saturation.

\subsection{Red blood cell aggregation}

\subsubsection{Physiology of red blood cell aggregation}

Human and most other mammalian RBCs tend to coalesce face to face with each other and form cylindrical structures that resemble stack of coins known as rouleau [6]. The rouleaux can grow larger in size with recruitment of more RBCs and form a three dimensional structure called

clusters. Figure 1-2 below shows human RBCs aggregated (AG) to form representative rouleaux and clusters.

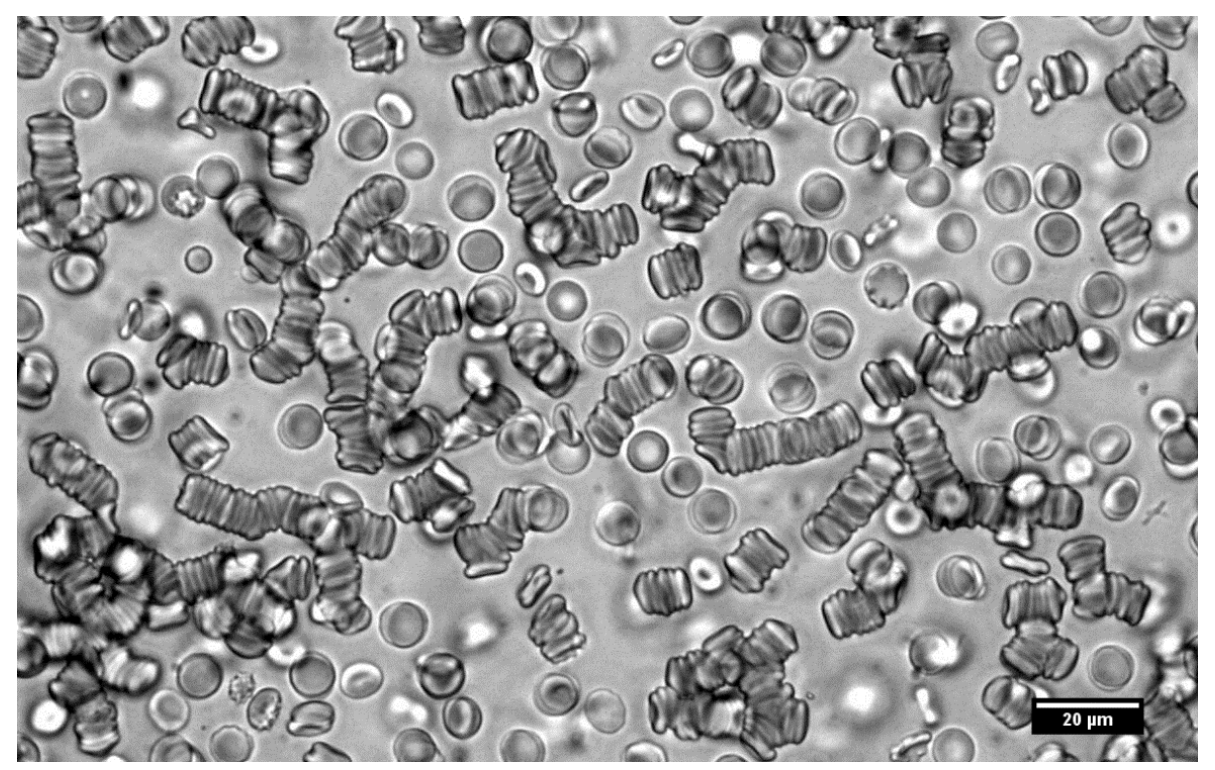

Figure 1-2: AG human blood, showing formation of rouleaux and clusters.

The forces causing aggregation are relatively weak and aggregates can be easily dispersed into non aggregated (NA) individual cells due to application of mechanical or fluid flow shear forces. Unlike blood coagulation, the plasma protein fibrinogen remains soluble in plasma during in vivo 
aggregation formation. Aggregation is a reversible process in the body under normal physiological conditions.

Aggregation is an influential factor in determining hemorheological properties. During low shear rates, typically present in the post capillary venules, RBCs aggregate and increase blood viscosity. However the forces causing aggregation are weak and the aggregates disperse under high shearing conditions in blood flow. This change in blood viscosity under high shearing rate is known as the shear thinning effect of blood [6].

RBC aggregation is mainly known to occur in athletic species and is almost nonexistent in sedentary species [6]. For athletic species aggregation can be beneficial in maintaining homeostasis since the change in blood viscosity under low and high shearing rates can regulate resistance to flow in microcirculation. During periods of high oxygen demand the blood flow to certain organs can increase by a significant amount. Capillary blood pressure is determined by the pre (arteriolar) and post (venular) blood pressure ratio. During increased tissue metabolism the arteriolar resistance (and blood pressure) falls because of vasodilation that increases blood flow. If the venular pressure were to remain the same the capillary pressure would be expected to increase. However maintaining a constant capillary pressure is crucial to ensure proper tissue perfusion, since the perfusion rate is driven by the difference between capillary and interstitial fluid pressures. To prevent this, shear thinning of blood results in venous resistance drop during high flow/high shearing conditions, ensuring capillary pressure does not change as the arterioles dilate. Hence aggregation, in conjunction with regulation of vessel diameter, acts as an auto regulatory mechanism of maintaining blood pressure at the micro circulatory level [6] under different blood flow rates. Aggregation has also been shown to increase axial migration of RBCs in flow due to decreased resistance to elastic compression by forming aggregates [6], [12], [13]. 


\subsubsection{Mechanism of RBC aggregation}

The aggregation process is determined by the balance between the forces that promote and oppose aggregation. The disaggregating forces are readily understood. They arise from mechanical shearing, RBC membrane strain opposing deformability and electrostatic repulsion due to a net negative RBC surface charge density.

Two theories have been proposed to explain the dynamics of aggregation: the bridging theory and the depletion theory. The bridging hypothesis assumes that when two RBC surfaces with molecules already adsorbed are brought close together, the free end of the molecules get adsorbed into the adjacent $\mathrm{RBC}$. This results in a cross linking between the RBC surfaces. Due to high deformability of the surfaces it is suggested that the RBCs will rotate and maximize bridging area of contact and produce rouleaux [6]. The depletion mediated aggregation model is currently the most widely accepted theory for aggregation [6], [14], [15]. When an RBC is present in a polymer solution, its surface produces a less maximized configurational entropy (i.e. the polymers near the RBC surface cannot occupy certain locations or orientations due to presence of the surface) and if this loss in configurational entropy is not balanced by the adsorption energy between the polymers and the surface (which causes polymer-surface adhesion), a layer around the surface develops in which the polymer concentration is depleted. If two such RBCs come close enough to each other causing their depletion layers to overlap, the osmotic pressure difference between the intercellular polymer-poor zone and the bulk phase causes the solvent to be displaced into the bulk phase. This minimizes the polymer-poor solvent regions between the cells increasing configurational entropy. As a result of this depletion an attractive force between the two RBC surfaces develop causing them to adhere. 
Currently it has also been suggested that the depletion model may be the initial mechanism by which the RBCs adhere and align and as time progresses the bridging of polymers due to adsorption can increase the inter-surface bond strength. A novel study using atomic force microscopy used single cell force spectroscopy of two adhering RBCs in DEX-70 solution to calculate the magnitude of the attractive forces due to the depletion theory. It was measured to be about $14-23 \mathrm{pN}[16]$.

\subsubsection{Factors affecting red blood cell aggregation}

\subsubsection{Cell and suspending medium properties}

The formation of aggregates depends on both the RBC membrane surface properties (charge, elasticity) and suspending medium properties [6]. It is important to distinguish between RBC aggregation and aggregability. While aggregation refers to the rate of formation and the strength of bond for the reversible rouleaux in any suspending medium, aggregability represents the formation kinetics and strength in a defined medium. Hence aggregability is a measure of the intrinsic capacity (a cellular property) of subpopulations of RBCs to aggregate. It is normal to find large range of RBC aggregability values between different healthy donors. The mammalian biconcave shape is a prerequisite for the formation of aggregates and any deviation from this shape due to changes of osmotic pressure, free radical attack, or formation of echinocytes markedly reduces aggregation.

\subsubsection{Aggregation and hematocrit}

Aggregation begins when random movement of RBCs in suspension brings them within certain proximity [17]. For low HCT, an increase in HCT results in increased frequency of close 
contacts or RBCs and thus higher extent and rate of aggregation [6], [18]. The initial phase of the aggregate formation seems to be independent of HCT where the cells aggregate to form a mean rouleaux length. The time taken to reach this critical length is however dependent on HCT. A higher HCT leads to a decreased critical time [19]. The concept of critical length can be understood in terms of the reactive area available. Until a certain number of cells have aggregated the rouleaux wall reactive area is much less than the area of the faces. However after the critical length has been achieved a pattern of aggregation based on columnar unit attachments appears, further enhancing aggregation. The second phase of growth is accelerated by a higher HCT [20].

The aforementioned studies were all done at low HCTs where the limiting factor to aggregate formation is the number of RBCs. Hence the decrease in aggregate formation time and increase in aggregate size is observed with increase in HCT. However as HCT keeps increasing further, the aggregate size decreases[6], [21]. An increase in number of RBCs can be seen as having the effect of decreasing the number of macromolecule available in solution per RBC. The forces promoting aggregation have strong dependence on the macromolecule concentration. Thus the size and kinetics of aggregation begins to have an inverse relationship with further increase in HCT [18].

\subsubsection{Role of macromolecules: Dextran}

The presence of macromolecules like fibrinogen can enhance RBC aggregation in autologous plasma. Fibrinogen is the most discussed plasma protein for inducing aggregation. It is an acute phase protein that may increase in concentration of $150-300 \mathrm{mg} / \mathrm{dl}$ to higher values during any form of an inflammatory response and for certain diseases. 
Rouleaux formation can also be induced by suspending RBCs in physiological solutions of macromolecules like dextran (DEX). DEX is one of the most widely used biocompatible macromolecules for aggregation studies. DEX has been used since mid-1940s in relation to hemorheological applications. The strength and extent of the aggregation produced depends both on the molecular weight of the DEX used and its concentration in solution [6].

In this thesis DEX with molecular weight of $70 \mathrm{kDa}$ (DEX-70) with $3 \% \mathrm{w} / \mathrm{v}$ concentration will be used to study aggregation of RBC because the highest level of aggregation is achieved at that concentration for human blood as shown in Figure 1-3. M1 is an unit of measuring aggregation in Myrenne aggregometer (Myrenne $\mathrm{GmbH}$, Roetgen, Germany), this device uses optical aggregation detection techniques.

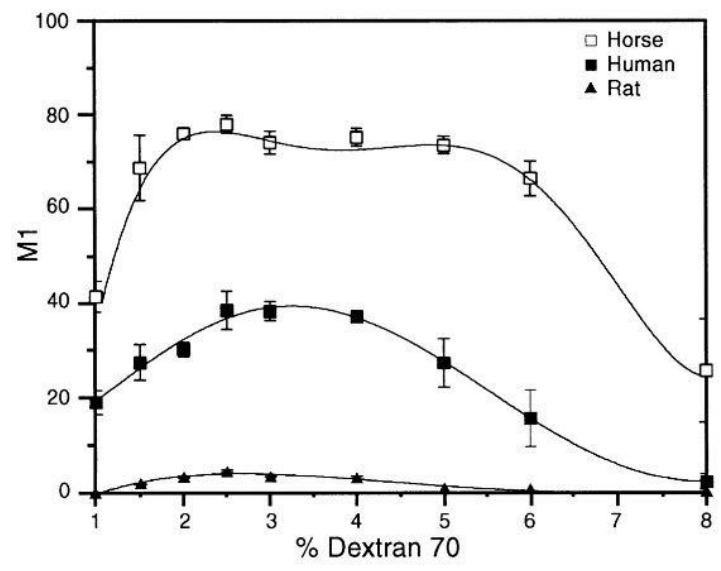

Figure 1-3: Relation between DEX-70 at different concentrations and amount of aggregation induced [6].

\subsection{Pathology of red blood cell aggregation}

Hyper-aggregation has detrimental effects on micro perfusion [23] and oxygenation of tissue [24]. Abnormal increase in aggregation levels can arise from any surgical procedure requiring extracorporeal circulation of blood or from any pathophysiological conditions that induce 
inflammation, affect circulatory or metabolic functions [25], [21]. The following paragraphs summarize some of the conditions that are either caused due to RBC aggregation or result in enhanced RBC aggregation.

The acute phase reaction is an extensive, nonspecific response of an organism to local or systematic disturbances. This results in increased production of positive acute phase proteins by the liver (like fibrinogen), and is a general mechanism in inflammatory response. Inflammatory response can be triggered by infections, tissue injury, trauma or immunological disorders.

In patients with various acute or chronic infections elevated acute phase response has been shown to correlate with increased RBC aggregation, forming larger aggregates and more of them in number than normal controls [26]-[30].

During sepsis or septic shock RBC aggregation may be altered due to both increased acute phase proteins and alterations to $\mathrm{RBC}$ aggregability. Atherosclerosis is an inflammatory disease with elevated levels of fibrinogen present. It is suggested that increased RBC aggregation due to increased fibrinogen can actually play an active role in progression of atherosclerosis by interfering with endothelial function [31]. Diseases like rheumatic arthritis are characterized by chronic inflammation and elevated RBC aggregation. Assessing the extent of aggregation is part of clinical evaluation of the progression, monitoring and treatment of the disease. Any kind of tissue injury may lead to an immunological response that may expose RBCs to free radicals altering the aggregability to favor formation of aggregates. Aggregability is altered because the RBC surface charge density is lowered. Also blood exposure to a non-physiological environment, like extracorporeal circulation during surgical procedures or implantation of foreign assisting devices, may introduce mechanical trauma to RBCs and enhance aggregation. 


\subsection{Current red blood cell aggregation quantification techniques}

\subsubsection{Erythrocyte sedimentation rate}

The sedimentation of RBCs is partially described by Stoke's law; a law that describes the force of viscosity on a small sphere moving through a viscous fluid. An RBC suspended in a medium has three main forces acting on it. The force favoring settling is the RBC weight. Forces opposing settling are buoyancy of the medium and the viscous drag. The viscous drag is proportional to the surface area of the RBC. But when RBCs combine to form aggregates, the surface area of the rouleaux formed is smaller than the sum of the surface areas of the individual RBCs. Hence the viscous drag on the rouleaux is reduced, producing an enhanced settling rate. HCT plays a significant role in determining the settling rate. The HCT alters aggregation kinetics and the settling process (which makes sedimentation a HCT and aggregation dependent process). The increased number of cells in the volume produces more interaction between the cells to hinder settling. This produces an inverse relation between HCT and settling. It is known however that blood sedimentation process is more complicated and Stoke's law is an over simplified way to describe erythrocyte sedimentation rate (ESR). The relation between aggregation and HCT is further discussed on pages 9 and 17.

The general procedure of measuring ESR is to suspend a certain volume of blood with premeasured anticoagulant in a standard dimension tube. After one hour the height of the RBC plasma on top of the RBC sediment is read. It is an invasive method requiring blood withdrawal. ESR also provides no information on the time course of the aggregation process and a decreased HCT due to anemia may falsely produce higher aggregation result due to enhanced settling. ESR varies from person to person depending on gender, age and other factors. The upper limit on 
settling is $10 \mathrm{~mm} / \mathrm{h}$ for men and $12 \mathrm{~mm} / \mathrm{h}$ for women. When adjusted for HCT, ESR correlates well with aggregation and is a technique of choice when other methods fail due to a very high level of aggregation.

\subsubsection{Low shear viscometry}

At low shear rates RBCs aggregate to increase viscosity and when shear rate is increased the RBCs disaggregate and deform to align themselves in the direction of the flow resulting in low viscosity. Once the cells have fully disaggregated and deformed a further increase in shear rate produces no further changes in viscosity. Correspondingly a suspension of modified RBCs with no deformability shows no change in viscosity with applied shear rate since ridged cells cannot form aggregates and increase viscosity. Also RBCs in buffer are not able to aggregate due to lack of macromolecules in suspension phase. This shows clear dependence of blood viscosity on RBC aggregation. Low shear viscometry uses this viscosity dependent relation at low shear rates to correlate with the extent of aggregation in the RBC suspension measured.

Viscometric measurements are vulnerable to several artifacts. A moderate decrease in RBC deformability may increase low shear viscosity even when there may not be any aggregation present. The plasma proteins are surfactants and can form a layer at interfaces affecting measurements. At low shear rates blood may sediment, altering local HCT or producing biphasic flow that can vitiate the data.

\subsubsection{Microscopic observations and image analysis}

Direct observation of blood samples has been a basic method to detect aggregation since the nineteenth century. Fabry [22] studied RBC aggregation using microscopic images reported that 
the shape and size of aggregates depend on the properties of the suspending medium and the HCT. The study reported using direct observation that increase in HCT in the range 10 to $50 \%$ results in slower sedimentation and decrease in aggregate size. Figure 1-4 shows the optical images obtained in this study [22].

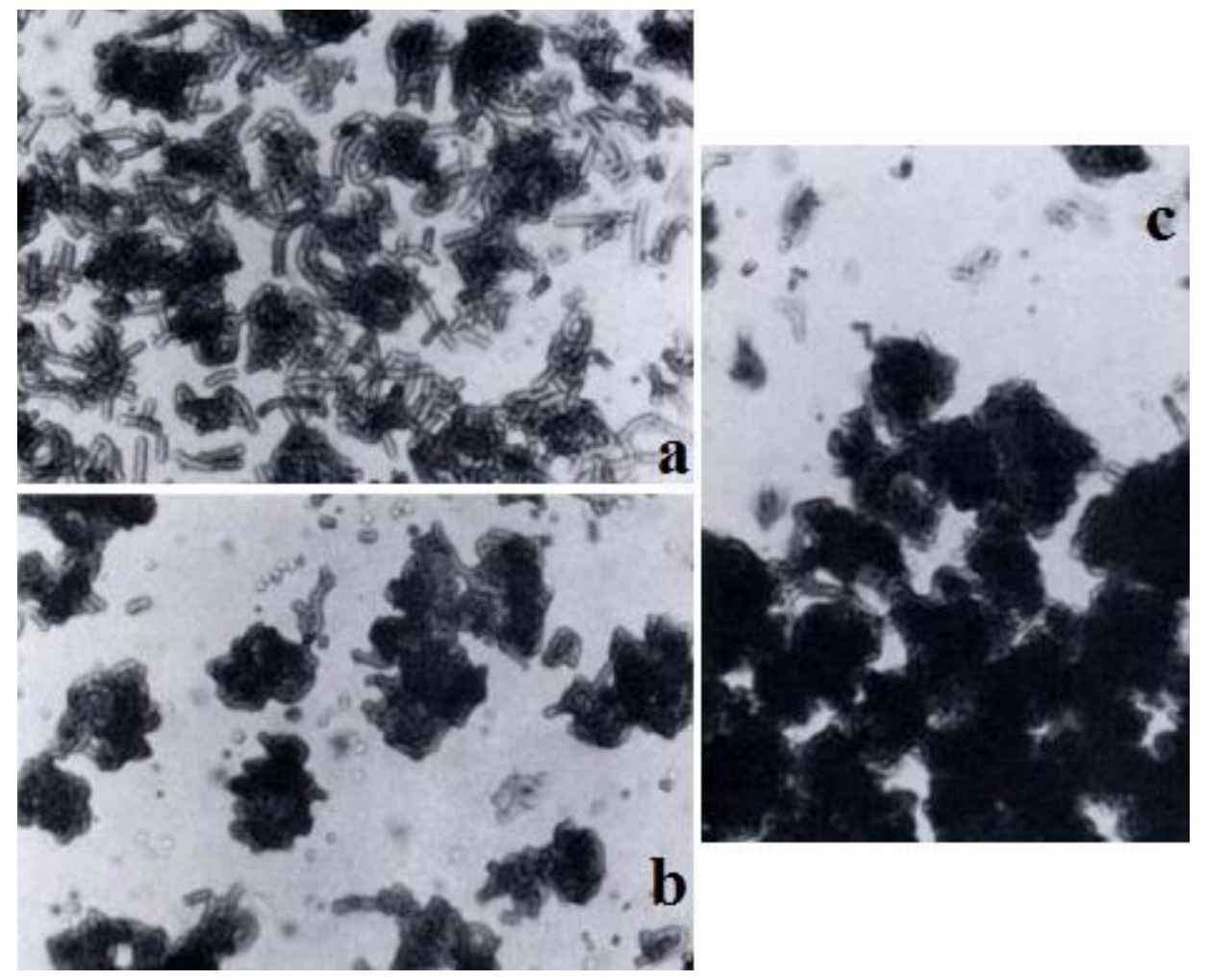

Figure 1-4: RBCs were suspended in autologous plasma to induce aggregation. (a) Shows RBC rouleaux formation in suspension, (b) uniform spherical clusters forming and (c) packed clusters sedimenting. Modified from [22].

Chien and Jan [32] quantified data obtained from microscopic observations of RBC suspensions to assess aggregation. The advantages of microscopic image analysis is the direct observation of aggregate size in terms of number of RBC per aggregate and realistic estimation of the extent of rouleaux formation compared to the indirect methods. Quantification of RBCs per aggregate in different shearing show that aggregates disperse at high shearing conditions [33]. Figure 1-5 
shows the results of image analysis in the flow chamber where the number of RBCs per aggregate was derived by dividing the projected area of an aggregate by that of a single cell. The thresholded images are shown along with the histogram of cells per aggregate. At shear stress of $0.125 \mathrm{dyne} / \mathrm{cm}^{2}$ the cells formed large rouleaux networks, and at $0.5 \mathrm{dyne} / \mathrm{cm}^{2}$ smaller rouleaux aligned themselves along the flow axis. At a shear stress of $2 \mathrm{dyne} / \mathrm{cm}^{2}$ the cells are mostly disaggregated as the histogram peaks at 1.5 cells per aggregate.
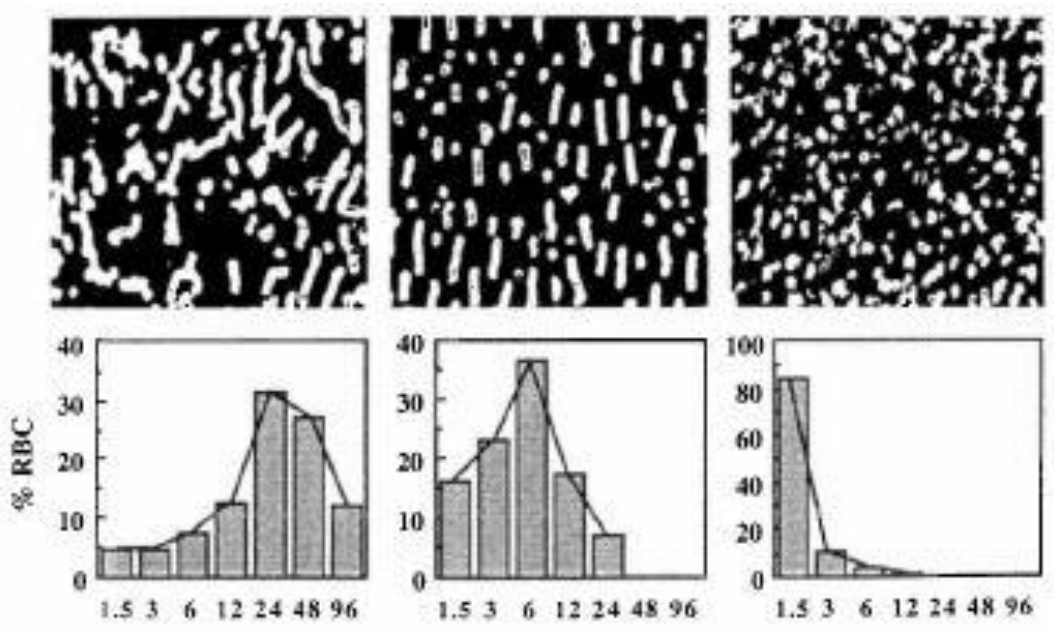

AGGREGATE SIZE (No. of RBC/Aggregate)

Figure 1-5: Human blood suspended in 10\% HCT with 0.5\% DEX-500, under different shearing conditions from left to right $0.125,0.5$ and 2 dyne $/ \mathrm{cm}^{2}[33]$.

Another study using 10\% HCT washed RBCs to monitor the size of aggregates in solution of $3 \%$ w/v DEX-70 reported the formation of clusters with about 100 or more cells in it [34]. The percentile distribution of RBCs in clusters containing certain number of RBCs was about $80 \%$ of the cells are in an aggregate containing $100 \mathrm{RBCs}$ or smaller and $20 \%$ of the cells are in aggregates with size of $10 \mathrm{RBCs}$ or smaller. 
With advances in automated image and video processing programs, intravital microscopy [35], [36] can be regarded as the closest, invasive (due to the need for removal of skin or application of special steps make skin more permeable to photons) in vivo methodology used for aggregation monitoring. Direct observation can also be used to quantify aggregation at very low or high levels of aggregation. For low aggregation levels the indirect methods may not be sensitive enough to detect any aggregation. For high aggregation levels it may be not possible to disaggregate the sample completely at the beginning of measurements which is a requirement for all studies quantifying some physical parameter correlated with changes in aggregate formation. In this thesis microscopic images from $A G$ and NA samples are used to quantify the validity of the aggregating protocol.

\subsubsection{Photometric methods}

\subsubsection{Optical aggregometry}

Light propagation in blood is mainly governed by the state of RBCs. Factors like HCT, alignment of RBCs and their aggregation status are all known to alter the blood optical properties [37]. Light beams are either absorbed or scattered from RBCs. In cases where the beam does not encounter an $\mathrm{RBC}$ in its path it may be transmitted through the blood film. In flow the RBCs elongate to align along the flow axis, leaving more gaps between the cells. When RBCs aggregate they form large structures with increased gaps of plasma or suspending medium between them. This allows for increased transmission and decreased reflection. Thus light transmission and backscattering is a function of the gaps between the cells or aggregates and can be used to monitor RBC aggregation. 
In optical aggregometry, blood is sheared at high rates to disaggregate all the RBCs and upon cessation of the shearing, light transmission or reflection through blood is recorded with time. Light reflection is dominated by the behavior of RBCs close to the surface exposed to the optical source whereas the transmittance properties of blood depend on the population of RBCs present in the entire path. The resulting intensity versus time graphs are called syllectograms and are analyzed to quantify the level of aggregation [6]. Syllectograms provide information about the time course of aggregation and the yield strength of the aggregates. The aggregation process has a fast and a slow changing phase. The first phase where the light intensity changes quickly is about 10 seconds and during the second phase there is a gradual continuation of the change. The shear rate, just sufficient to prevent $\mathrm{RBC}$ aggregation, measured in optical aggregometers corresponds to the magnitude of forces holding the RBCs together and is called the disaggregation shear rate.

Although photometric methods are widely used to quantify aggregation, this mode of aggregation detection has significant drawbacks. Firstly the measurements obtained from one instrument cannot be directly compared to another. Different results are obtained due to the fact that aggregation is dependent on the geometry of the suspension-loading chamber of the

measuring instrument. Secondly the measurements are not directly translatable to in vivo conditions [38].

\subsubsection{Optical properties of blood and effect of dextran}

Administration of DEX changes optical properties of blood. The changes lead to increased light penetration in blood and is termed optical clearing [39]. RBC aggregation due to DEX allows increased transmission through gaps of plasma between RBC aggregates (with plasma scattering 
coefficient being about 3 orders of magnitude less than RBC scattering). Also the higher anisotropy factor related to the increased size of aggregates produces more forward scattering [40].

A flattening factor is used for estimating whole blood absorption coefficient when derived from measurements using the same amount of hemoglobin molecules homogeneously distributed in solution [41]. The reduction of bulk absorption coefficient comes from unattenuated areas where the photons pass through the suspension of RBCs without encountering any absorber [41]. As a consequence, the transmitted light intensity is higher than it would be if all the hemoglobin were uniformly dispersed in the solution. The same concept may be applied to the absorption coefficient of $\mathrm{AG}$ and NA blood. In AG blood the inter aggregate spacing between the RBC aggregates is larger than the inter cellular spacing amongst the more uniformly dispersed single RBCs. Thus RBC suspensions with no aggregation will have higher absorption coefficient and AG RBC suspensions will have a reduced absorption coefficient. This effect is known as the 'Sieve effect' [42]. However this is not to be confused with the 'local' increase in absorption where an aggregate is situated [43]. An aggregate packs hemoglobin densely per unit volume when compared to normal blood at physiological HCT. The absorption coefficient is proportional to HCT or concentration of hemoglobin.

The other factor influencing optical clearing is refractive index matching. A predominant source of optical scattering in blood is the difference in refractive indices of blood plasma $\left(n_{p}=1.345\right)$ and RBC cytoplasm $\left(n_{c}=1.405\right)$ [39]. When DEX is added the plasma refractive index is raised to a value closer the RBC cytoplasm and hence the scattering coefficient of blood is reduced. Decreased scattering increases optical energy density and increases the light penetration depth in the illuminated volume of the sample. Optical scattering has a complex nonlinear relation that 
saturates with HCT higher than $10 \%$, due to violation of the assumption of independent scattering [44].

Aggregation and sedimentation are both critical components regulating optical properties of blood, since they change the absorber distribution and consequently also the fluence distribution in a suspension.

\subsubsection{Optical methods to measure blood sedimentation}

Sedimentation has also been studied by monitoring the changes in light transmission of blood [45]. During sedimentation the suspension of RBCs separated into two layers, a clear upper layer of plasma and a lower layer containing plasma, single RBCs and aggregates. The sedimentation process showed a sigmoid curve over time, with three distinct phases. Phase one was characterized by slow fall of the position of the interface between the two fractions, phase two by fast fall and phase three displayed an asymptotic behavior. The time duration of the slow fall was attributed to the time taken for the aggregates to form and pack. The delay in commencement of the fast fall suggested that RBCs did not settle rapidly unless a certain aggregate size was achieved. During the fast fall phase the suspended RBC aggregates that have reached a stable characteristic spherical volume were sedimenting and the bottom sediment layer was packing further. Phase three represented further continued packing of the sediment layer. Strzelecka et al [46] reported sedimentation of RBCs resuspended in $4 \% \mathrm{w} / \mathrm{v}$ suspension of DEX-70 at $40 \%$ HCT which revealed the sigmoid nature of sedimentation as shown in Figure 1-6.

The deposit always contained some amount of plasma and in the packing process this amount decreased. The HCT of the sediment grew during phase one and two reaching a steady value, higher than the initial HCT of the whole suspension, at the end of the packing phase three. 


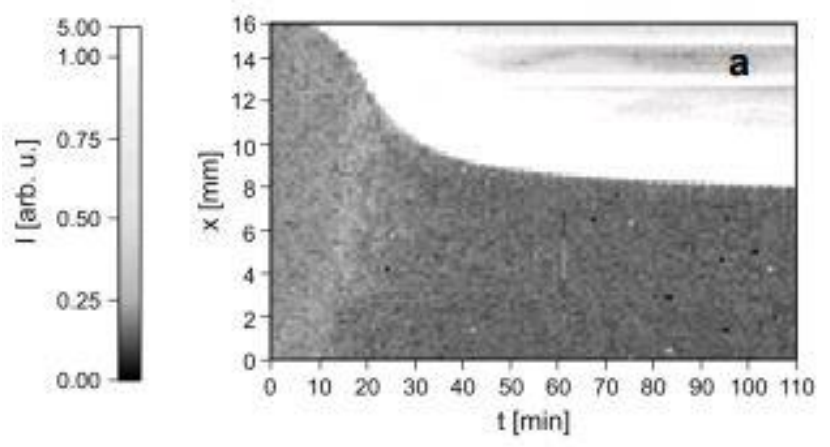

Figure 1-6: The intensity of light transmitted through the sample of RBC suspension in $4 \%$ w/v DEX-70 as a function of time $t$ and altitude $\mathrm{x}[46]$.

\subsubsection{Ultrasonic blood characterization}

The radio frequency (RF) US signal backscattered from tissue conveys information about the tissue microstructure. RF data obtained from the tissue is converted to B mode (brightness mode) images by the process of envelope detection. B mode images convey information about tissue echogenicity. Changes in blood echogenicity due to aggregation have been well studied [47], [48].

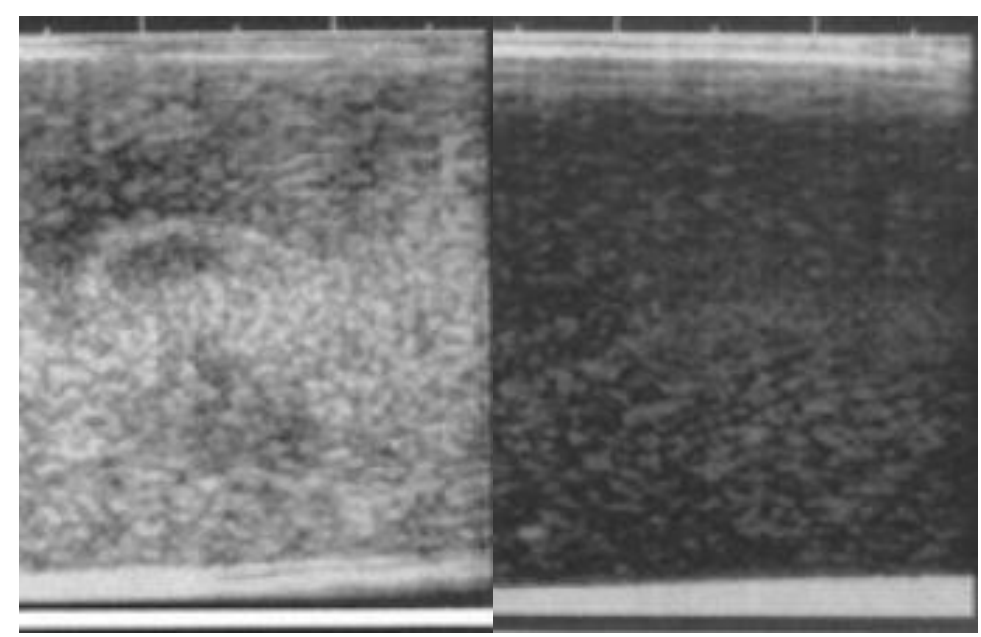

Figure 1-7: B mode images showing blood echogenicity from AG whole porcine blood (left) and NA porcine RBC (right) [47]. 
The left image in Figure 1-7 is obtained from whole porcine blood in a chamber with static conditions (where aggregates can form) and the right image is from washed RBCs suspended in saline solution (to suppress aggregation). From the B mode images it can be seen that the AG sample was more echogenic than the NA sample [47]. Thus aggregation is known to enhance echogenicity of blood.

However in the process of envelope detection, information contained on a fine scale in the RF data is lost. Analyzing RF data directly form selected region of interest (ROI) enables analysis of these fine features which are dependent on tissue microstructure. After compensating for the imaging system dependencies by using signal from a reference spectrum (such as that from a planar US reflector) the normalized power spectrum obtained from the RF data and expressed in $\log$ scale $(\mathrm{dB})$ can be fit to a straight line [49].
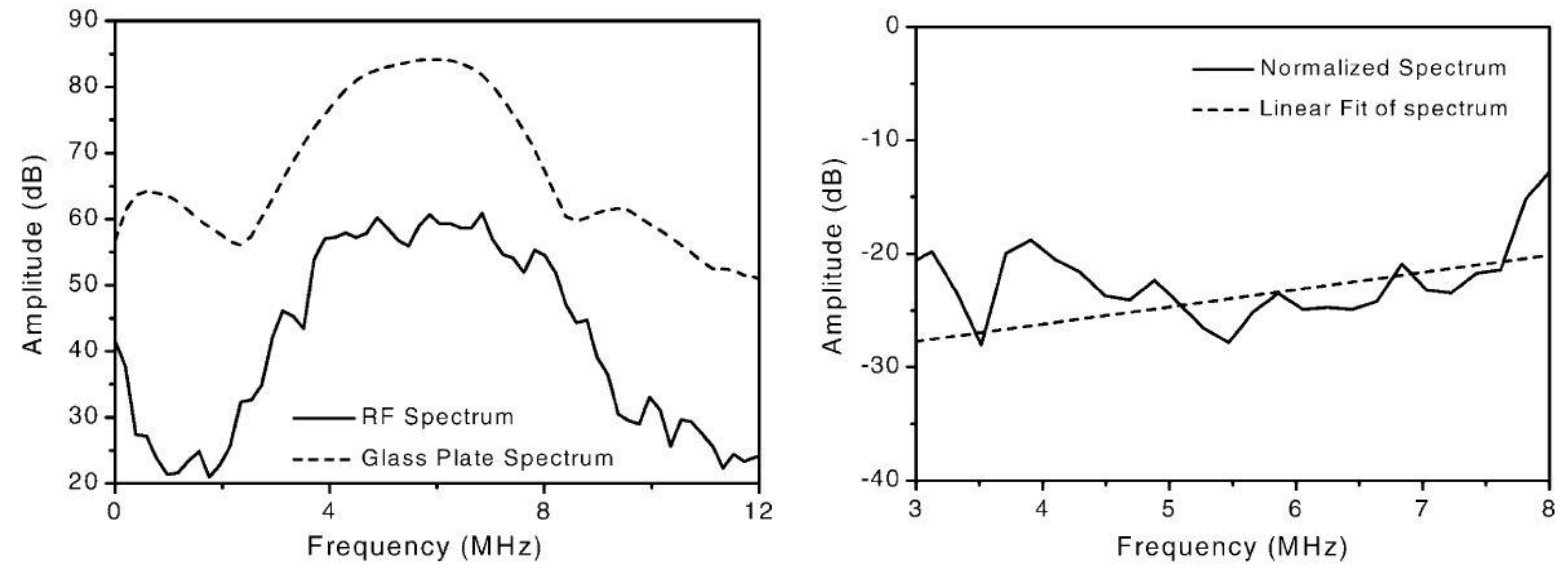

Figure 1-8: (Left) power spectrum obtained from tissue (solid) and a glass plate as a planar US reflector (dashed). (Right) tissue power spectrum after normalization [49]. 
The slope of the linear regression fit to the normalized spectrum (right image in Figure 1-8) is called the spectral slope (SS) and is measured in $\mathrm{dB} / \mathrm{MHz}$. The $\mathrm{y}$-value of the linear fit at midfrequency of the frequency range analyzed is called the midband fit (MBF) and is measured in dB. SS and MBF are spectral parameters which are often extracted in quantitative ultrasound (QUS) for tissue characterization [49]. When the diameters of the scatterers are less than a tenth of the insonifying US wavelength, the nature of the scattering is in the Rayleigh regime. With an increase in size of the scattering sources (so that the Rayleigh regime is no longer applicable [21], the SS becomes less positive [3]. The MBF increases with an increase in the size and concentration of the scatterers [3], [79].

Another spectral parameter the backscatter coefficient (BSC) can also be used to reveal microstructure and composition of tissue [50], [51]. In ultrasonic blood characterization the dense packing of scattering RBCs introduces a nonlinear relation between the BSC and HCT. The nonlinearity arises from an increase in spatial correlation amongst particles with increasing crowding. The correlation factor (also known as the packing factor) is a correction for the increase in destructive phase interference in coherent field arising from the spatially correlated scatterers [21]. The BSC peaks at a certain HCT for a particular US frequency and then drops with further increase in HCT [52]. In addition to HCT, blood aggregation also influences the BSC. The structure factor size estimator with attenuation estimation (SFSAE) model [4], [21] , [25] was developed to introduce a second order data reduction model which predicts the dependence of BSC and its deviation from the Rayleigh assumption due to RBC aggregation. The two parameters that are obtained from this model are packing factor $\mathrm{W}$ which relates to the variation of BSC with $\mathrm{HCT}$, and D the isotropic diameter of the aggregate (expressed in number of RBCs). A recent study [53] conducted on diabetic patients reported that, D measured from the 
cephalic vein correlated with the strength of aggregation parameter $\gamma_{\text {th }}$ (minimum shear rate required to prevent aggregation) measured in vivo by laser-assisted optical aggregometer.

The WBC count in the microcirculation may increase significantly under inflammatory conditions. This may add artifacts to RBC aggregation detection technique since WBCs present in insonification area may add to the scatter. In venous flow conditions where WBCs adhere to the walls for extravasation, US scattering may be more pronounced. Several studies [54], [55], [56] have shown the role of endothelial-leukocyte interaction in acute inflammation related ischemia reperfusion injury after a stroke, myocardial infarction or administration of coronary revascularization procedures [57]. Presence of inflammation mediated conditions like diabetes only enhance the WBC endothelial adhesion and rolling in venules [57]. Even though at low shear the WBCs are usually present near the vascular wall and the RBC aggregates at the central flow region, a method of in vivo investigation that has more control (using RBC and WBC absorption spectra) over distinguishing WBCs from RBCs is desirable.

While US can assess RBC aggregation in vivo, it generally suffers from the issue of poor contrast due to the similar acoustic impedances of different soft tissues and cannot provide functional information like the oxygenation state of RBCs.

\subsection{Current biomedical applications of photoacoustics}

Tissue is a highly scattering medium in the visible spectral range which limits penetration and degrades resolution at imaging depths more than $1 \mathrm{~mm}$. US pulse echo detection is based on mechanical property change in tissue. The weak contrast in soft tissue, due to similar acoustic impedances, can limit differentiation of structures. PA imaging can provide greater tissue 
differentiation and specificity because the optical absorption in different tissue types can vary by orders of magnitude depending on the spectral window chosen.

For PA imaging biological tissue is irradiated with nanosecond pulsed laser beam. The temporally confined optical absorption causes heat deposition and induces a local temperature rise [58] which causes thermoelastic expansion due to tissue elasticity and launches a sound or stress wave. The thermoelastic properties of some soft tissue allow for high signal to noise ratio (SNR) in the generated PA signals without causing any thermal damage. After propagating through the tissue the pressure wave can be detected using a passive (receive mode) ultrasonic transducer to map the optical absorption in tissue with US resolution: this form of PA is called acoustic resolution PA. In PA, the scattering of sound waves in biological tissue is 2-3 orders of magnitude less than light and can be typically ignored, however US attenuation due to frequency dependent absorption does present a resolution-penetration trade-off in PA. At a frequency of $30 \mathrm{MHz}$ the attenuation coefficient of blood is about $0.3 \mathrm{~dB} / \mathrm{cm}$ [59] but because of one way US propagation in PA, signal detection for up to several $\mathrm{cm}$ is possible. In this mode PA imaging depth is limited by photon diffusion not US attenuation.

Since PA combines the most compelling features of optical and US imaging, it has been used to obtain anatomical, functional, metabolic, molecular information in biomedicine. Different implementations allow PA to have high depth to resolution ratio of about 1/200 [58] for up to several centimeters in tissue which is not achievable in any other biomedical modality. PA is a nonionizing, nondestructive (depending on laser energies used), and noninvasive modality which provides structural and functional information with very good combination of resolution and penetration. 
PA imaging has been tested in many areas of biomedical research including cancer [60]. Monitoring cancer therapy by detecting changes in the hemoglobin concentration and $\mathrm{SO}_{2}$ of the tumor microvasculature [61] and obtaining high contrast images of prostate cancer [62] are some the areas where PA can be implemented. The ability to map structure, $\mathrm{SO}_{2}$ and flow characteristics (similar to conventional US power Doppler) of the vasculature makes PA imaging well suited to the assessment of tumors and other pathologies characterized by abnormalities in morphology and function of the vasculature. PA has been used in many applications including noninvasive imaging of breast, sentinel lymph nodes, skin, thyroid, eye, ovaries, and endoscopic imaging of gastrointestinal tract [60]. PA can also be used to image circulating tumor cells to determine metastases [63], [64]. In addition, tissue temperature mapping [65] and monitoring of therapeutic applications such as high intensity focused US treatment [66] can be monitored by PA due to the PA signal generation dependence on tissue temperature. Characterization of atherosclerotic plaque [67], tissue characterization including bone [68], blood, and soft tissue are also areas where PA is being explored as a possible mode of investigation. Optical resolution PA has been used to image at the cellular level [69]. PA imaging is developing at a rapid rate due to its unique ability to exploit both optical and thermoelastic properties of tissue.

\subsection{The photoacoustic effect}

The PA effect was first reported by Alexander Graham Bell in 1880 [70]. In the PA effect tissue irradiated with short laser pulse absorbs light energy which is converted to energy deposition per unit volume leading to a pressure rise, thermoelastic expansion and subsequent emission of a broadband acoustic pressure wave [71]. For efficient generation of PA signal the laser pulse width should be short enough so that thermal diffusion and stress relaxation of the absorber during heat deposition can be neglected. 
The PA pressure from an optically thin fluid sphere of radius $a$ with sound speed $c_{s}$ and density $\rho_{s}$ surrounded by a transparent fluid with sound $\operatorname{speed} c_{f}$, density $\rho_{f}$, irradiated by light intensity $I_{0}$ sinusoidally modulated by a frequency $\omega$ can be expressed as a function of the dimensionless frequency $\hat{q}=\omega a / c_{s}$, at large radial distance $r$ from the sphere as Equation (1-1) [72]

$$
p_{f}(\hat{q})=\left(\frac{i \mu_{a} \beta I_{0} c_{s} a}{C_{p} \hat{r}}\right) \frac{(\sin \hat{q}-\hat{q} \cos \hat{q}) e^{i k_{f}(r-a)}}{\hat{q}^{2}[(1-\hat{\rho})(\sin \hat{q} / \hat{q})-\cos \hat{q}+i \hat{\rho} \hat{v} \sin \hat{q}]}
$$

where $\beta$ is the isobaric thermal expansion coefficient, $C_{p}$ is the isobaric heat capacity per mass, $\hat{\rho}=\rho_{s} / \rho_{f}$ is the dimensionless density ratio, $\hat{c}=c_{s} / c_{f}$ is the dimensionless speed of sound ratio, $\hat{r}=r / a$ and $k_{f}$ is the wave number in the fluid medium. The term in brackets for Equation (1-1) is responsible for the amplitude of the signal and the second part characterizes the spectral variations. The frequency content of the PA signal from an absorber depends on the absorber shape, symmetry, dimensions, ratio of sound speed and density relative to the surrounding medium.

The temporal width of the PA signal from a single target is proportional to its size, so a small absorber has more high frequency components in the PA signal spectrum. Figure 1-9 illustrates that a larger absorber has more power at lower frequencies when compared to a smaller absorber. The PA MBF increases with absorber size and concentration [73]. Concentration affects the increase in MBF more distinctly than size. This can be explained by the concentration dependence of the absorption coefficient which is related exponentially to the optical energy deposition per unit volume. Whereas the radius is linearly proportional to the amplitude as showed by the terms in brackets in Equation (1-1) and indirectly affects the amplitude via the complicated dependence 
of the second part of the equation. Plot of PA spectral power in $\mathrm{dB}$ for single fluid spheres with different sizes using Equation (1-1) are shown in Figure 1-9 (a). The lack of substantial change in MBF at higher absorber size further reinforces the stronger concentration dependence.

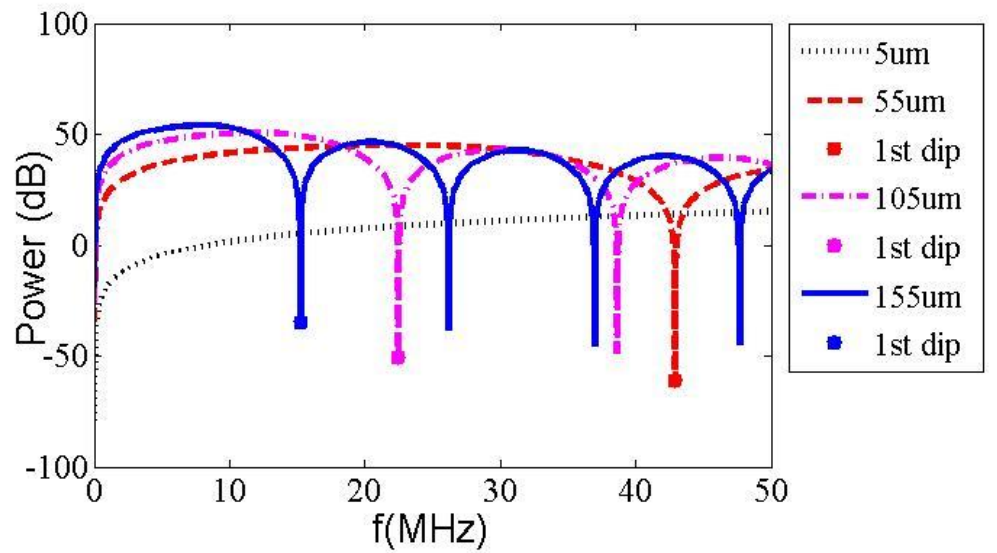

Figure 1-9: Theoretical PA signatures for spherical droplets with density and sound speed properties similar to RBC and different diameters.

Distinct spectral dips are obtained at frequencies where the numerator of Equation (1-1) goes to zero, which satisfies the condition $\tan (q)=q$. For absorbers with different diameters the characteristic first dip will be dependent on the ratio of the diameter and sound speed. The method of spectral matching uses the location of dips to derive the size of imbedded absorbers in tissue [74], [75]. This method works if the particle concentration and size are such that dips are observed within the detecting transducer bandwidth from the ensemble PA signal.

\subsection{Photoacoustic tissue characterization}

In biomedical applications the more realistic scenario is derived using the linear superposition of signals from many such small spherical absorbers [76]. Saha [77] showed the PA crosssection exhibits a $f^{2}$ dependence in the small particle regime unlike ultrasound which exhibits $f^{4}$ 
dependence. Also near the tissue boundaries the signals add up coherently and produce a strong boundary build up [77], [78]. Kumon et al [79] experimentally showed that the MBF varied significantly between cancerous and non-cancerous tissue. For experimental data the PA signal is normalized to a reference spectrum to remove the imaging system response.

\subsubsection{Using photoacoustics to assess RBC aggregation}

Saha et al [1] characterized the level of aggregation in blood using PA signal simulation. AG and NA blood was simulated in 2D by using Monte Carlo random sequential adsorption technique with the condition of no overlap among the cells. For NA cells HCT was varied from 6 to $50 \%$. For AG case HCT was chosen to be $40 \%$ and different levels of aggregation were simulated by changing the radius of the isotropic clusters from 7.57 to $15.39 \mu \mathrm{m}$. 


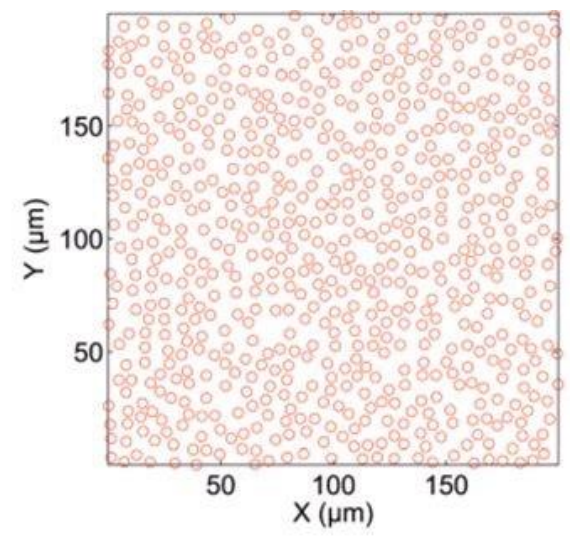

(a)
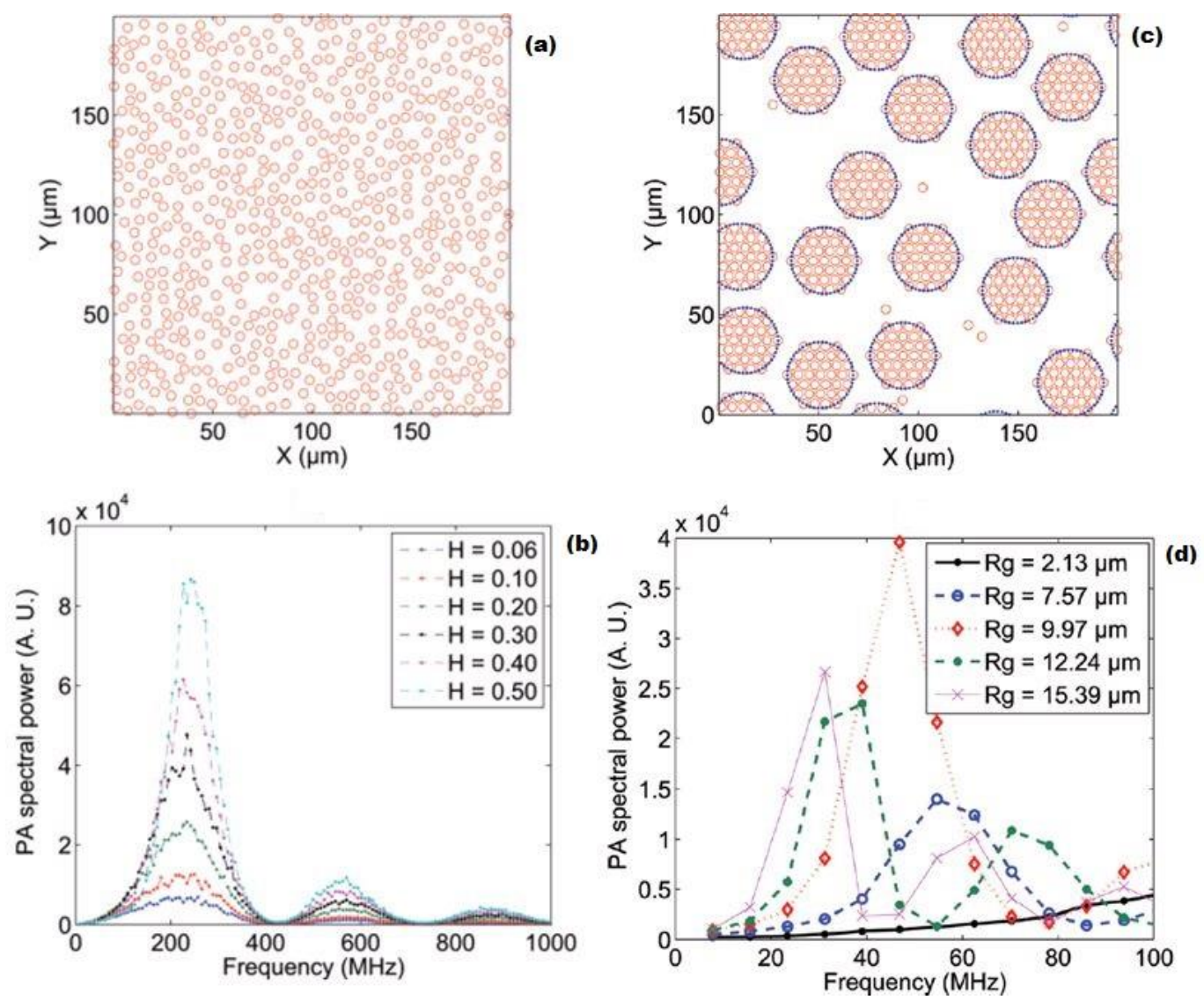

Figure 1-10: Sample representative of realizations of (a) NA and (c) AG RBCs radius of gyration $12.24 \mu \mathrm{m}$ simulated, (b) shows the change in monotonous rise in PA power spectra amplitude with increasing HCT for NA RBCs and (d) shows the changes in peak positions and amplitudes in PA power spectrum as a function of different levels of aggregation [1].

Figure 1-10 shows the distribution of $A G$ and NA samples and their PA power spectra. For the NA simulation it can be seen that with increasing HCT the amplitude of the power spectra increased monotonically. In the AG samples the first frequency minimum appeared at lower frequency as the cluster size increased. In Figure 1-10(d) the simulated power spectrum shows a trend of increasing MBF with increase in size; however the presence of spectral dips influences the MBF and SS depending on the analysis frequency range chosen. 
Hysi et al [2] experimentally demonstrated an increase in PA signal amplitude (SA) and mean spectral power with increased level of aggregation at $5 \mathrm{MHz}$ center frequency. The mean spectral power at maximum aggregation was $4.8 \mathrm{~dB}$ higher. The $\mathrm{SA}$ also increased with increase in HCT. Hysi et al [3] also reported change in SS with aggregation size, where the slope decreased by a factor of 7. The change in slope was observed for two different illumination wavelengths. However no significant variation in SS with HCT was reported. Figure 1-11 shows the PA SS and MBF changes for NA and AG RBCs.

(a)

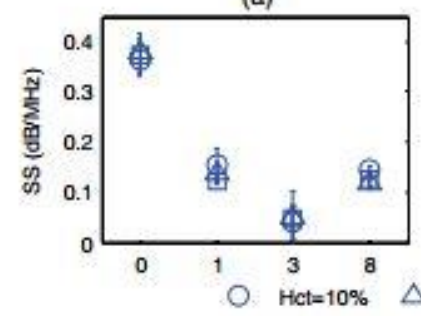

(c)

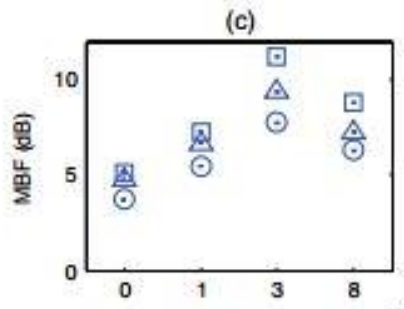

(b)

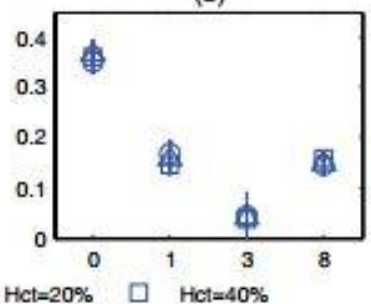

(d)

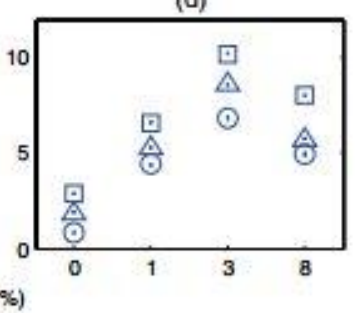

Figure 1-11: (a) PA SS for $750 \mathrm{~nm}$ wavelength, (b) PA SS for $1064 \mathrm{~nm}$ wavelength, (c) PA MBF for $750 \mathrm{~nm}$ wavelength, (b) PA MBF for $1064 \mathrm{~nm}$ wavelength. Error bars denote standard deviation of 20 PA signals and power spectra [3].

For all measurements the MBF was highest for the highest level of aggregation. MBF also increased by $1.2 \mathrm{x}$ for each doubling of HCT. The effect of aggregate size dependence on HCT was not observed in MBF changes. 


\subsubsection{Using photoacoustics to assess RBC oxygen saturation}

The hemoglobin absorption coefficient is dependent on its state. $\mathrm{HbO}_{2}$ and $\mathrm{Hb}$ have distinct spectral absorption coefficients (Figure 1-1) and this allows their concentrations and level of oxygenation to be detected using multi spectral PA analysis. Given that oxygen delivery is the primary function for RBCs, the ability to detect $\mathrm{SO}_{2}$ is a significant advantage of PA over US in vascular imaging. $\mathrm{SO}_{2}$ detection is based on multispectral PA imaging. The intensity of the PA signal is proportional to the absorption coefficient $\mu_{\mathrm{a}}$ of the absorber [80], and $\mu_{\mathrm{a}}$ depends on the

blood $\mathrm{SO}_{2}$ [3]. This relationship between PA signal and $\mu_{\mathrm{a}}$ can be used to determine blood $\mathrm{SO}_{2}$ by using Equation (1-2)

$$
S O_{2}=\frac{S A\left(\lambda_{2}\right) \varepsilon\left(H b, \lambda_{1}\right)-S A\left(\lambda_{1}\right) \varepsilon\left(H b, \lambda_{2}\right)}{S A\left(\lambda_{1}\right) \Delta \varepsilon\left(\lambda_{2}\right)-S A\left(\lambda_{2}\right) \Delta \varepsilon\left(\lambda_{1}\right)}
$$

Where $\lambda_{1}$ and $\lambda_{2}$ are two different illumination wavelengths, $\varepsilon$ is the molar extinction coefficient, $\Delta \varepsilon$ is the difference between the molar extinction coefficients of $\mathrm{HbO}_{2}$ and $\mathrm{Hb}$ for each wavelength.

Saha et al [76] reported the effect of $\mathrm{RBC} \mathrm{SO}_{2}$ using simulations. Results showed that PA signal changed as a function of the level of $\mathrm{SO}_{2}$ simulated. Using experimental methods Hysi et al [3] reported a higher $\mathrm{SO}_{2}$ for aggregates indicating impaired oxygen release from individual RBCs. The result is presented in Figure 1-12. The relation between $\mathrm{RBC}$ aggregation and $\mathrm{SO}_{2}$ was also measured by Tateishi et al [24] using optical methods. Tateishi suggested the reduced area to volume ratio of aggregates as one reasons for the reduced oxygen release. 


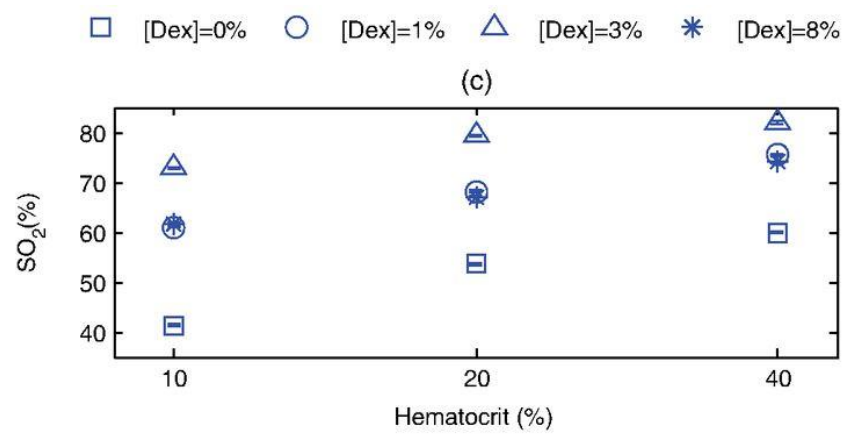

Figure 1-12: Experimentally measured PA SO2 for different levels of aggregation and HCTs of 10, 20 and $40 \%$. DEX-70 concentration of 3\% was most aggregating and DEX-70 0\% was non aggregating DEX-70 of concentration

$1 \%$ and $8 \%$ were moderately aggregating. Results were obtained using samples of washed porcine RBCs [3].

Jensen published [81] on the mechanism of release of oxygen from RBC in to the plasma. RBCs sense extracellular changes in $\mathrm{pH}$ and $\mathrm{PO}_{2}$ which is associated with tissue metabolism. Intracellular $\mathrm{Hb}$ oxygen affinity is affected by these changes in plasma and oxygen unloading is accordingly affected to optimize oxygen delivery. When aggregates form, the individual RBCs are confined by stagnant plasma and other RBCs. This impairs extracellular $\mathrm{pH}$ sensing and oxygen release from the individual RBCs and thus aggregates remain oxygenated.

\subsubsection{Using photoacoustics to assess blood sedimentation}

The relation between aggregation and sedimentation has been discussed in detail in the preceding sections. A study by Kinnunen [40] reported the pulse amplitude and arrival time using PA and concluded that the PA pulse arrival time can be used to detect RBC aggregate sedimentation velocity. In parallel with PA, optical coherence tomography (OCT) imaging was also employed to verify the PA method. The sedimentation velocity as function of HCT is plotted in Figure 1-13 using data from the publication. From the results it is evident that aggregation increases sedimentation velocity (for NA samples the velocity is $\sim 0.3 \mathrm{~mm}$ in 5 minutes and about $\sim 3 \mathrm{~mm}$ 
in 5 minutes for AG samples) by a factor 10 or more at $20 \%$ HCT. For a blood samples containing NA RBC suspensions the sedimentation is less than $0.08-0.24 \mathrm{~mm}$ during a 5 minute interval. And for AG samples the sedimentation ranges from 1.25-3.5 mm during the same duration.

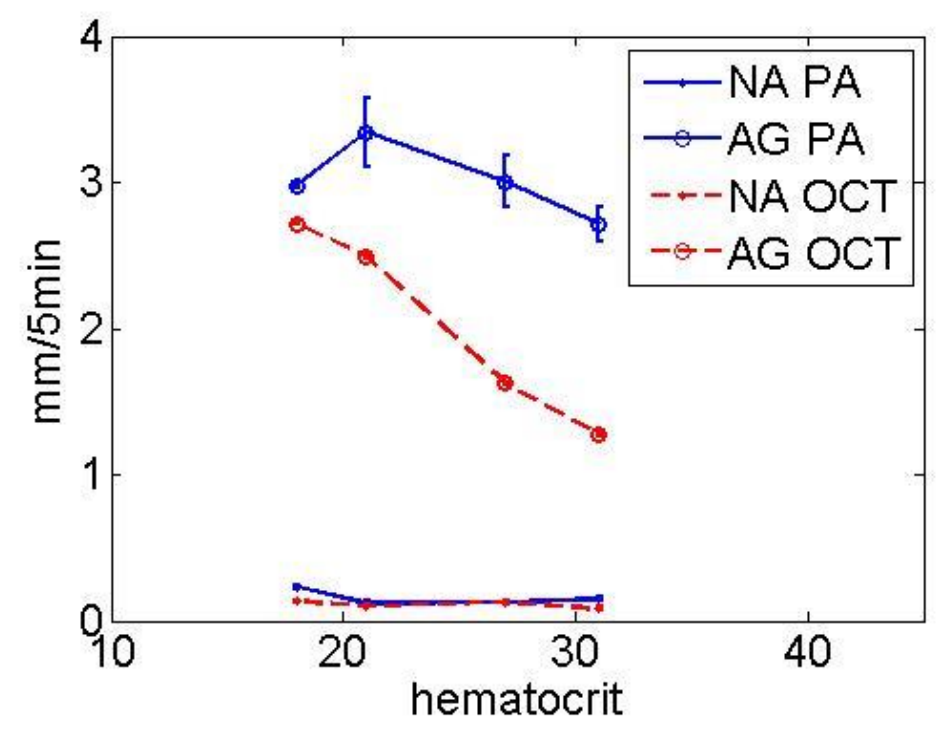

Figure 1-13: Estimation of sedimentation in 5 mins using PA and OCT measurement data points for NA and AG blood, using data from [40].

\subsubsection{Numerical simulation of photoacoustic signals}

Work on numerical simulation of PA signals using time resolved arrival time of sound waves generated by absorbing tissue at an array of pressure transducers have been reported in several publications [82], [83], [84]. Figure 1-14 shows the schematic [83] for an PA wave generation in an absorbing object. 


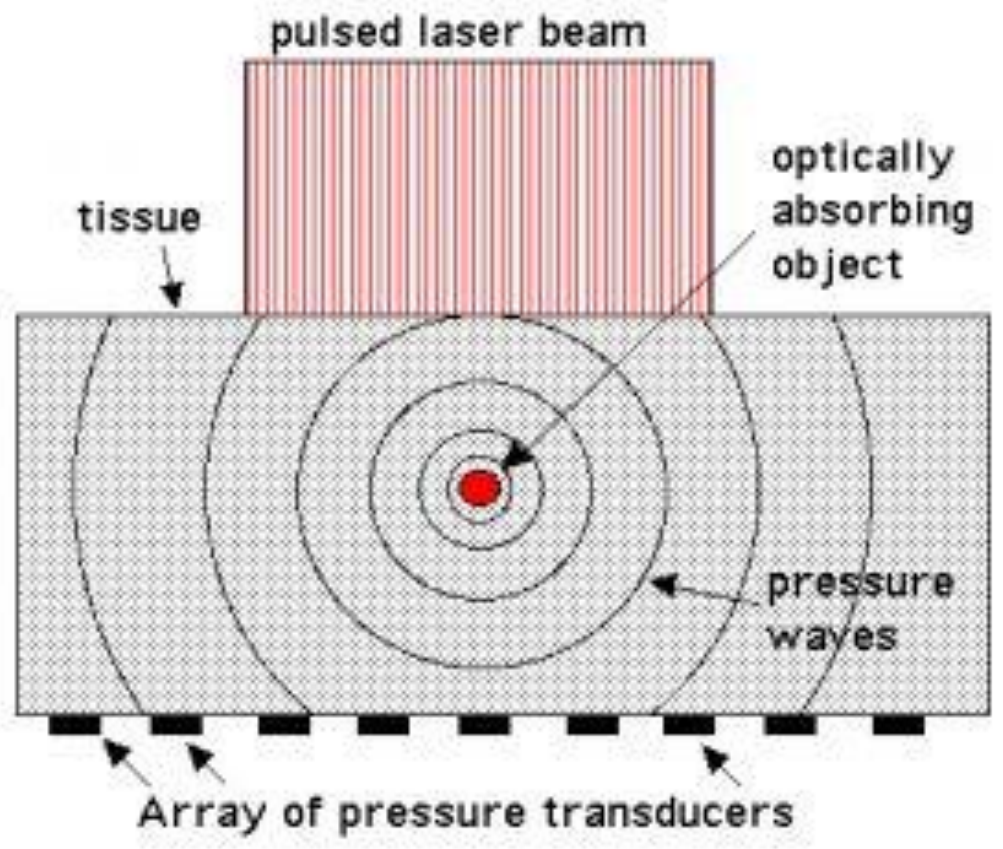

Figure 1-14: Schematic of a photoacoustic simulation [83].

The simulation uses Monte Carlo code to obtain the fluence in the irradiated volume. The fluence is then converted to energy deposited based on absorption profile and the subsequent time resolved pressure launch from the absorbing volume. The pressure wave is detected at an array of pressure transducers. Figure 1-15 shows the energy deposition profile for a spherical absorber depicted in red, and 3 detectors are placed the locations specified in the image. The PA pressure profiles detected by these detectors are shown in the image at the right in Figure 1-15 [83]. 


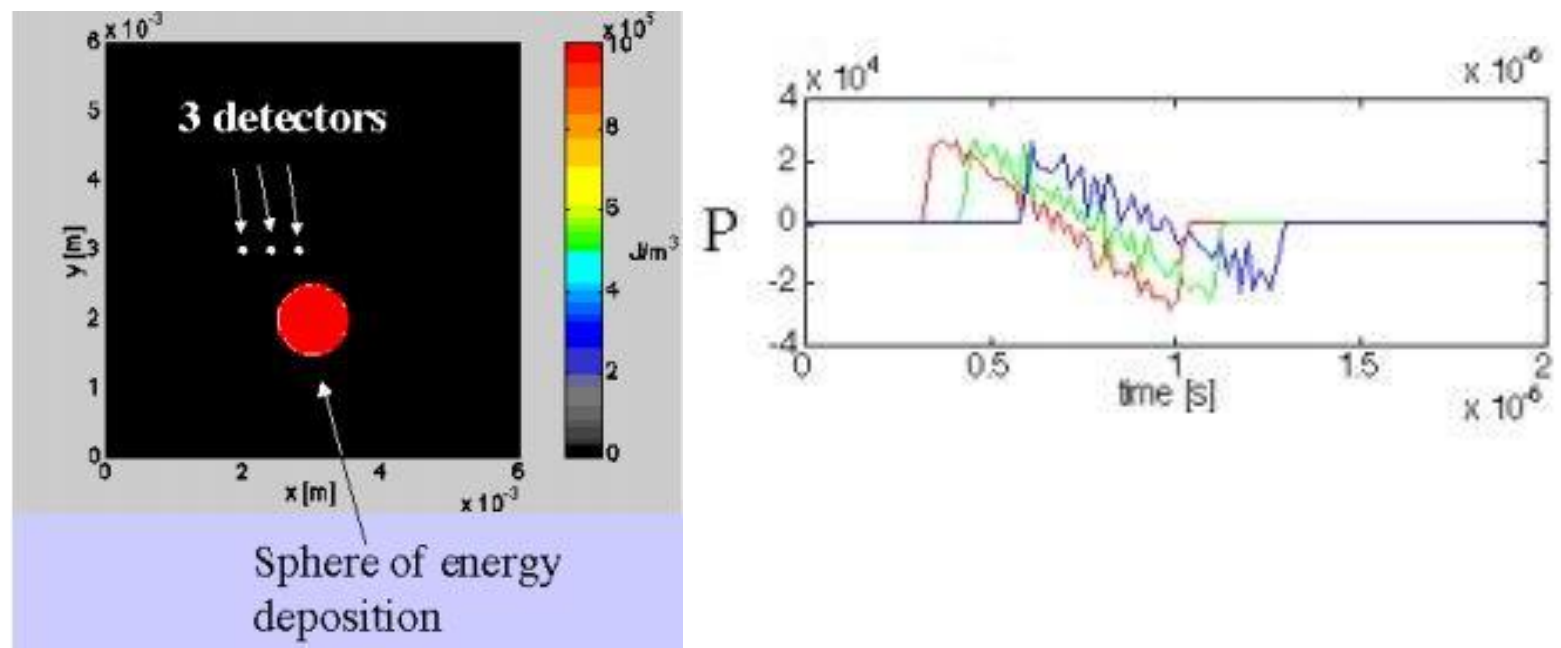

Figure 1-15: (Left) Energy deposition profile for an spherical absorber with the positions of 3 detectors specified, (right) corresponding PA A line signals from the three detectors [83].

Numerical simulation of PA signal using the energy deposition distribution has also been used to mode cerebral blood oxygenation. The resulting data from such simulation was shown to be in good agreement with the experimental results [84].

\subsection{Thesis hypothesis and objectives}

Based on the background presented in the introduction it is hypothesized that spectral parameters obtained from high frequency (>20 MHz) PA signals can be used to detect RBC aggregation.

The objectives are to:

a) Use microscopic image analysis techniques to quantify the level of aggregation artificially induced in RBCs using DEX-70.

b) Obtain high frequency PA experimental signals from AG and NA RBCs in vessel mimicking phantoms using a $25 \mathrm{MHz}$ US probe. 
c) Analyze time domain PA signal amplitude (SA), and implement QUS analysis to investigate how the spectral parameters (SS and MBF) change as a function of aggregation. 


\section{Chapter 2 : Methods}

\subsection{Blood sample preparation}

Whole human blood used for the experiments were obtained from Canadian Blood Services (CBS). The supernatant and the buffy layer were removed by careful aspiration after centrifuging at $1500 \mathrm{~g}$ for 5 minutes. Blood was first washed twice with DMEM by centrifuging at $1500 \mathrm{~g}$ for 5 minutes. After the second wash the DMEM was adjusted in the tube to make $70 \%$ packed RBCs [6]. Packed RBCs were added to Dulbecco's modified essential medium (DMEM) containing 10\% fetal bovine serum (FBS) (Sigma Aldrich, St. Louis, MO) solution to create three different final sample HCTs of 40, 20, and 10\%. Each of the HCT samples were aliquot in two. A 20\% w/v stock solution of DEX-70 (Sigma Aldrich, St. Louis, MO) and DMEM-FBS was added to one aliquot (AG) creating 3\% final DEX-70 concentration in the sample, with total volume of $1000 \mu \mathrm{L}$. To create 0\% DEX-70 DMEM-FBS sample (NA), only DMEM-FBS was added to the other aliquot making a final volume of $1000 \mu \mathrm{L}$. The experiments were conducted on three separate days to ensure repeatability. A total of six samples were produced per experiment. The following Table 2-1 shows the volume of each component added to create the samples. 


\begin{tabular}{lcccccc}
\multicolumn{2}{r}{} & \multicolumn{2}{c}{$\mathbf{2 0 \%}$ DEX DMEM-FBS } & \multicolumn{2}{c}{ DMEM-FBS } & \multicolumn{2}{c}{ Packed RBC } \\
\hline & $\mathbf{0 \%}$ & $\mathbf{3 \%}$ & $\mathbf{0 \%}$ & $\mathbf{3 \%}$ & $\mathbf{0 \%}$ & $\mathbf{3 \%}$ \\
$\mathbf{4 0 \%}$ & $\mu \mathrm{L}$ & $\mu \mathrm{L}$ & $\mu \mathrm{L}$ & $\mu \mathrm{L}$ & $\mu \mathrm{L}$ & $\mu \mathrm{L}$ \\
\cline { 2 - 6 } & 0 & 150 & 429 & 279 & 571 & 571 \\
$\mathbf{1 0 \%}$ & 0 & 150 & 714 & 564 & 286 & 286 \\
\hline
\end{tabular}

Table 2-1: Packed RBC, DMEM-FBS and 20\% DEX-70 in DMEM-FBS volumes added to create six samples with 40, 20, $10 \%$ HCTs and two DEX-70 concentrations of $0 \%$ and $3 \%$.

For the hemocytometer measurements, packed RBCs were resuspended at $1 \% \mathrm{HCT}$ in $0 \%$ and 3\% final concentration DEX-70 DMEM-FBS solutions. Table 2-2 shows the volumes of packed RBCs, DMEM-FBS and DMEM-FBS with DEX-70 added to create the samples with final volume of $1000 \mu \mathrm{L}$.

\section{0\% DEX DMEM-FBS DMEM-FBS Packed RBC}

\begin{tabular}{ccccccc}
\hline DEX & $\mathbf{0 \%}$ & $\mathbf{3 \%}$ & $\mathbf{0 \%}$ & $\mathbf{3 \%}$ & $\mathbf{0 \%}$ & $\mathbf{3 \%}$ \\
$\mu \mathrm{L}$ & $\mu \mathrm{L}$ & $\mu \mathrm{L}$ & $\mu \mathrm{L}$ & $\mu \mathrm{L}$ & $\mu \mathrm{L}$ \\
\cline { 2 - 7 } $\mathbf{1 \%}$ & 0 & 150 & 986 & 836 & 14 & 14 \\
\hline
\end{tabular}

Table 2-2: Volumes (in $\mu \mathrm{L}$ ) of packed RBCs, DEX-70 plus DMEM-FBS and DMEM-FBS added to create hemocytometer samples with $1 \% \mathrm{HCT}$ and $0 \%$ and $3 \%$ DEX-70 concentrations. 


\subsection{Hemocytometer image collection and blood aggregation assessment}

This method of analysis to check aggregation protocol was chosen because the experiment and analysis can be carried out without any special laboratory equipment. Using DEX-70 to aggregate RBCs is a well-studied method in literature and an established protocol was used [85].

\subsubsection{Sample loading and microscopic image acquisition}

In the hemocytometer $10 \mu \mathrm{L}$ of $1 \%$ HCT RBC suspension in 0\% DEX-70 DMEM-FBS was administered and incubated in a $37^{\circ} \mathrm{C}$ humidified chamber for 15 minutes. The RBC suspension was then imaged by a microscope (Model-CX-41, Olympus America Inc., Center Valley, PA, USA). Brightfield images were obtained by the camera (Model Retiga 2000R, QImaging, Surrey, BC, Canada) under 40x magnification and saved for analysis. The same procedure was repeated with $10 \mu \mathrm{L}$ of $1 \%$ HCT RBC suspension in 3\% DEX-70 DMEM-FBS solution. All measurements were repeated on three separate days and prepared from the same packed RBCs used for PA phantom studies.

\subsubsection{Image analysis}

The 8 bit grayscale, 1600x1200 pixel images were analyzed with Fiji [86], an image processing package primarily build for research analysis in the life sciences field. Some of the rouleaux and single cells (resting on the edge) were selected to produce an axial intensity profile. The intensity profile was used to count the number of cells in the rouleaux. Single cells were measured to estimate the width of a single cell on its side.

The unsharpmask filter with a weight of 0.9 and 10 pixel radius was applied to the images to preserve the cell shapes which may have been blurred due to focusing through the 
hemocytometer. The images were thresholded to separate the RBCs and the suspending medium background. Thresholding was done using the Otsu method [87]; this method minimizes the interclass variance of brightest and darkest pixels. After thresholding the binary watershed processing was applied to separate individual cells or aggregates (collectively referred to as units) from one another. The watershed algorithm [88] works by calculating the Euclidian distance map of each pixel and then finding the ultimate eroded points which were then dilated till the edge of the particle was reached or till the edge touched another particle region.

After thresholding and segmenting, particle analysis was performed to calculate parameters like area and circularity for each unit. A total of 279, 315 and 306 units were analyzed from the AG sample images obtained during experiment 1, 2 and 3 and for NA samples a total of 598, 520 and 535 units were analyzed. To ensure only RBCs were counted, particles with an area below 15 $\mu \mathrm{m}^{2}$ were excluded from the analysis. The NA areas were binned in increments of $10 \mu \mathrm{m}^{2}$. The bin with maximum numbers of units was taken to be the representative size of an $\mathrm{RBC}$ resting on its circular side.

The number of RBCs in rouleaux in the AG samples was calculated by profiling the axial intensity for rouleaux in the grayscale image. A line was manually drawn across the axis of the cylindrical shaped rouleaux, and the intensity profile along that line was plotted. Drops in intensity profile indicated edge of an RBC. A total of five rouleaux from each AG image were analyzed using the intensity profile method. The area of each unit chosen for intensity profiling was divided by the respective total number of cells counted in the unit, and thus the average side projection area of an $\mathrm{RBC}$ was calculated. 
Units were sorted by their circularity to determine if the projected area was from an RBC resting on its flat side or from rouleaux. Areas of all units with circularity above and equal to 0.885 were divided by the flat RBC area projection. Units with circularity below 0.885 were divided by area projected by an RBC on its edge. Thus the total numbers of RBCs per unit were calculated. Data for number of cells per unit was binned in increments of one cell per unit.

\subsection{Photoacoustic data acquisition and analysis}

\subsubsection{Blood vessel mimicking phantom preparation}

The phantoms were made by using $1 \% \mathrm{w} / \mathrm{v}$ agar and PBS solutions, and refrigerating overnight to solidify. Cylindrical holes were freshly incised into the phantoms just before the experiment. This created a blood vessel mimicking lumen with $1 \mathrm{~mm}$ diameter in the phantom. During experiments the RBC samples were freshly prepared and injected in to the phantom vessel. Both ends of the vessel were then sealed with melted agar solution. The phantom was rotated several times to homogenize the sample and minimize RBC sedimentation as much as possible before

placing it in room temperature PBS bath for coupling with the transducer. Figure 2-1 below shows the phantom setup in the PBS bath for the experiments. 


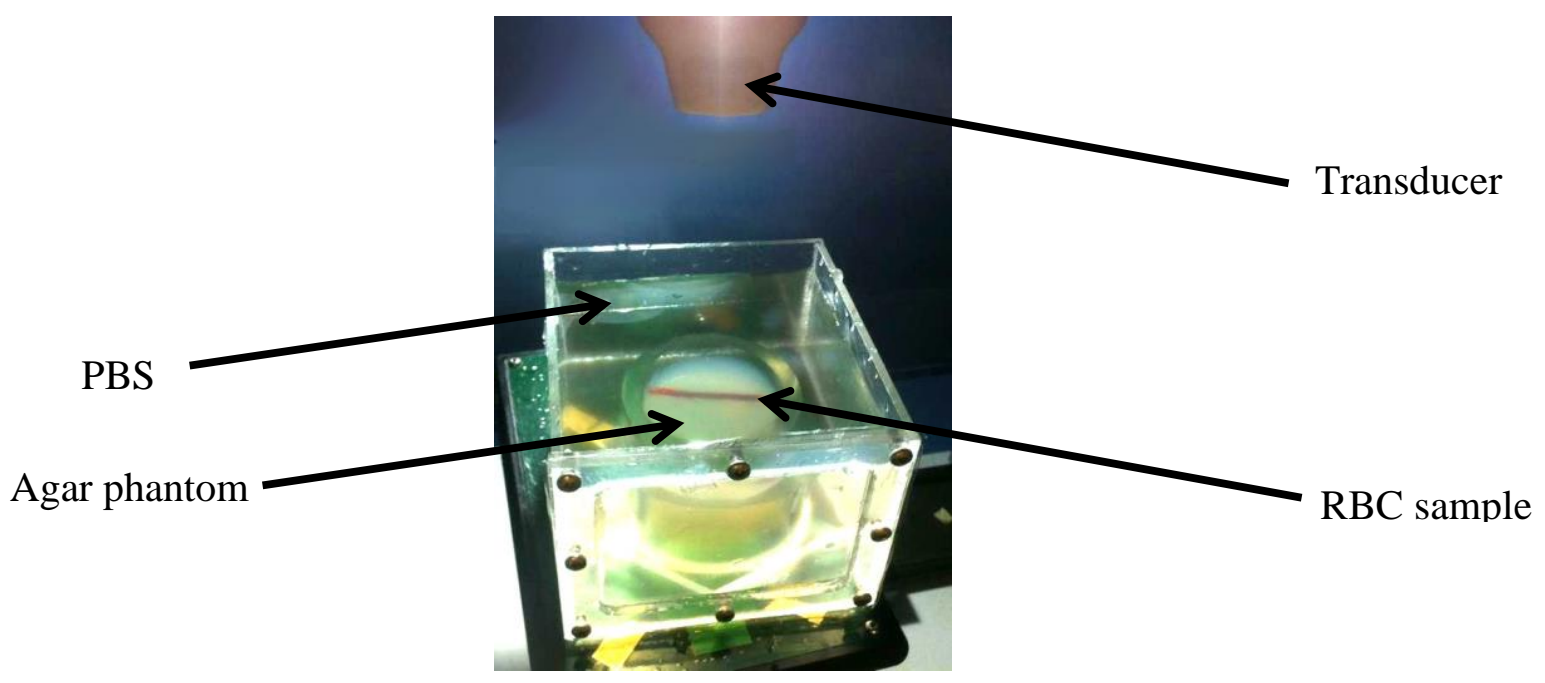

Figure 2-1: Agar phantom setup with blood sample inside and prepared for imaging with the LZ 550 transducer at the top.

The blood vessel was imaged along its length producing B mode images of the vessel which was $14 \mathrm{~mm}$ wide and $1 \mathrm{~mm}$ in diameter. The RF data were saved for offline analysis. Figure $\mathbf{2 - 2}$ shows the schematic of how the phantoms were set up to acquire PA measurements.

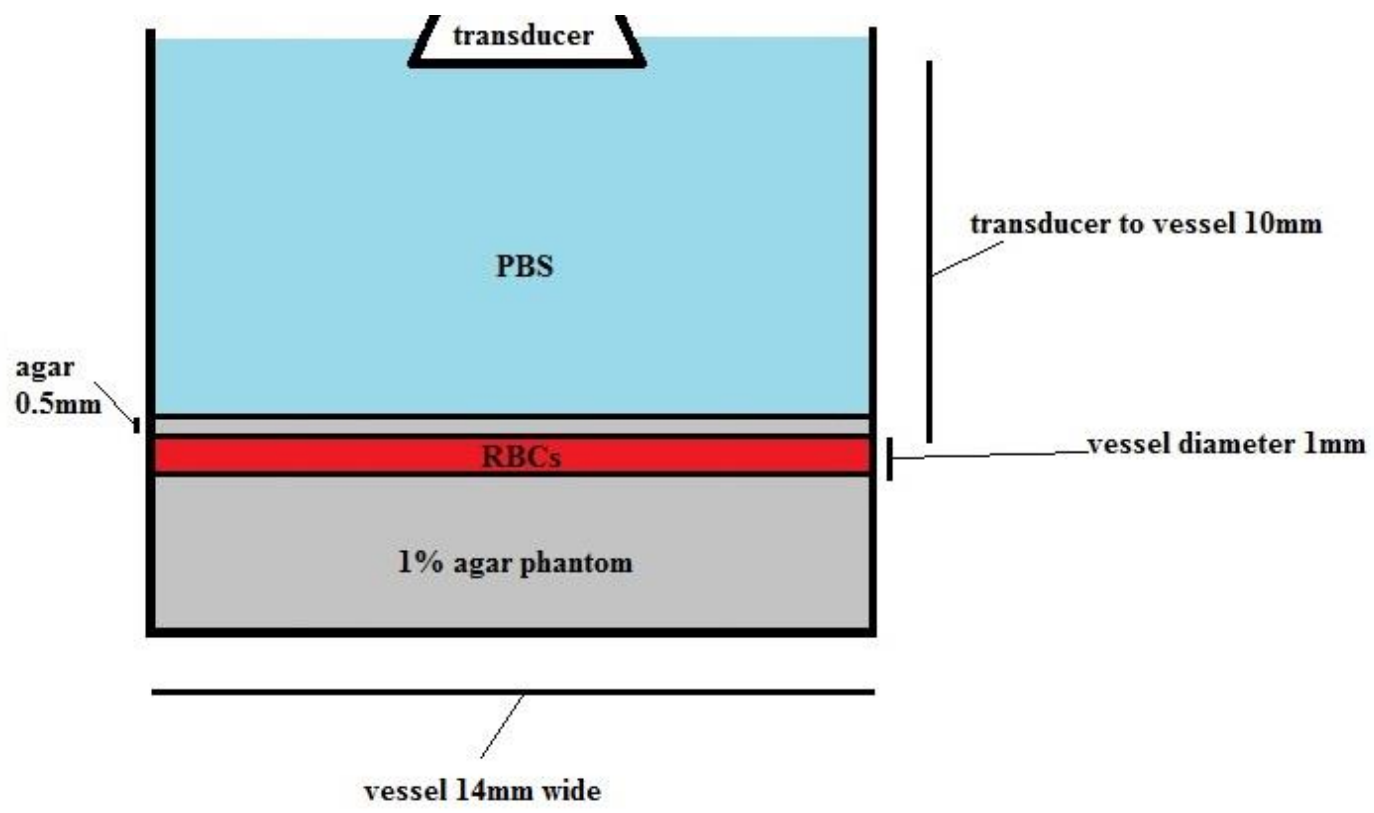

Figure 2-2: Schematic of the phantom setup for PA measurements. 
The vessel to transducer distance was $10 \mathrm{~mm}$. the vessel was incised about approximately 0.5 mm below the top surface of the agar phantom.

\subsubsection{VEVO 2100 / LAZR Photoacoustic imaging system}

The VEVO 2100 / LAZR (FUJIFILM Visual Sonics, Toronto, ON, Canada) imaging system was used to acquire both US and PA data from the phantoms. The transducer used was LZ550 with linear array of 256 elements. The system consisted of three structural modules: Vevo LAZRTight was a steel cabinet that housed the phantoms and the PA transducer, Vevo LAZR cart housed all the laser optical system and the Vevo 2100 US cart housed the software, electronics, manual controls and monitor that were used during both PA and US data collection. Figure 2-3 below shows the imaging system with the modules and the transducer.

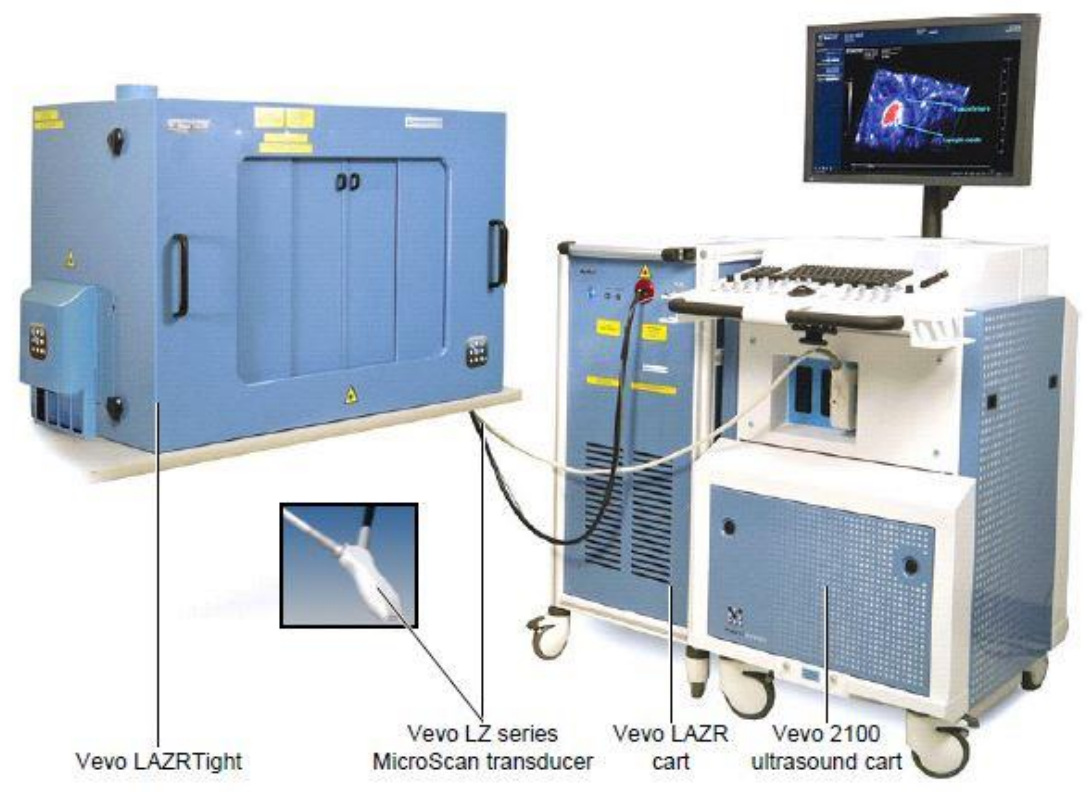

Figure 2-3: The Vevo LAZR Photoacoustic imaging system. The four main components of the imaging system are the Vevo LAZRTight, LZ series transducer, Vevo LAZR cart and the Vevo 2100 US cart with the monitor. 
The optical source for PA imaging were two $(20 \times 1.25) \mathrm{mm}^{2}$ fiber bundle termination areas located on either side of the linear array transducer. The laser source was a Nd:YAG system with optical parameter oscillator which produced photons with wavelengths from $680 \mathrm{~nm}$ to $970 \mathrm{~nm}$. The pulse repetition rate was $20 \mathrm{~Hz}$, with $10 \mathrm{~ns}$ pulse width and pulse energy of $30 \mathrm{~mJ}$. The laser beams intersected at an axial depth of $10 \mathrm{~mm}$ from the transducer and at an angle of $\pm 30^{\circ}$ to the imaging plane. The axial, lateral and elevation resolutions of the transducer created a resolution

volume of $(40 \times 95 \times 154) \mu \mathrm{m}^{3}$, which was about $585 \times 10^{3} \mu \mathrm{m}^{3}$. The digitization frequency of A/D converters were $32 \mathrm{MHz}$. PA RF was digitized using the quadrature [89] 2x technique with a sampling frequency of $64 \mathrm{MHz}$. To reconstruct the RF signal the samples are interpolated by a factor of 16 , providing a reconstruction sample rate of $1.024 \mathrm{GHz}$.

\subsubsection{Blood phantom data acquisition}

The top boundary of the phantom blood vessel was placed at a depth of $10 \mathrm{~mm}$ from the transducer. US focal zone was set at the same depth. US images were acquired at $40 \mathrm{MHz}$ and 42 $\mathrm{dB}$ gain. PA imaging was conducted at 750 and $850 \mathrm{~nm}$ laser wavelengths and the PA signal gain was set at $70 \mathrm{~dB}$. In total $20 \mathrm{PA}$ frames were acquired per phantom at a rate of 5 frames per second. The PA data was collected from a $14 \mathrm{~mm}$ wide and $15 \mathrm{~mm}$ deep B mode plane that included the lateral view of the blood vessel. The laser was pulsed four times and 64 sequential A lines were acquired at each pulse to produce a B mode image (one frame) comprised of 256 A lines. The beamformed US signal along with the pre beamformed PA RF were stored. Screenshot images of the phantoms were also stored. 


\subsubsection{Gold film measurement}

After each experiment a $200 \mathrm{~nm}$ thick gold film was placed on top of an agar phantom with no blood vessel and was positioned at $10 \mathrm{~mm}$ axial depth from the transducer. The gold film is a broadband PA signal emitter and the features in the measured PA power spectrum from a gold film represent the transducer response to a broadband signal [3]. The US and PA signal from the gold film was recorded and stored with same settings as the blood phantom measurements except for the gains, US gain was set to $0 \mathrm{~dB}$ and PA gain was at $50 \mathrm{~dB}$. 20 frames were acquired at 5 frames per second for both modes and wavelengths. Figure 2-4 shows a typical transducer response obtained from the gold film signal. The central peak is at $25 \mathrm{MHz}$ with a $-6 \mathrm{~dB}$ bandwidth of 10-48 MHz.

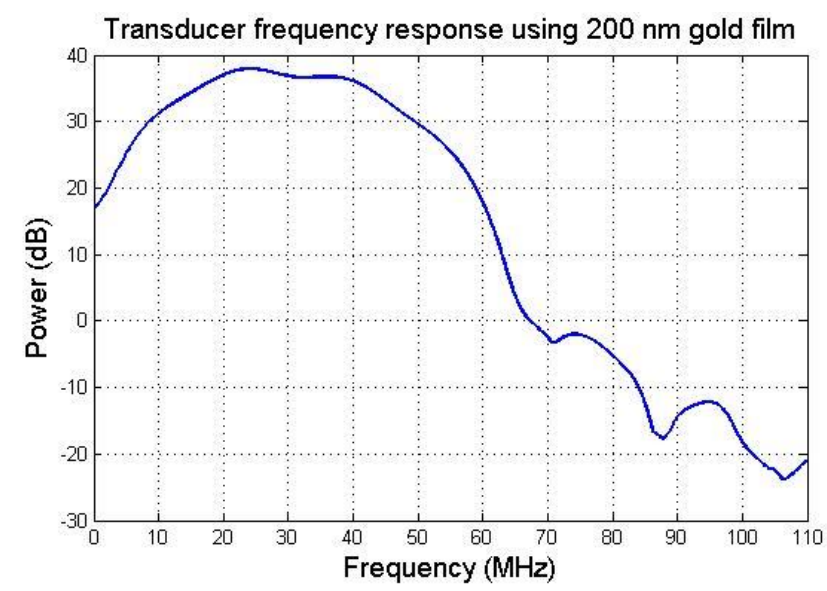

Figure 2-4: Typical PA gold film frequency response for LZ-550 transducer.

\subsubsection{PA data Analysis using MATLAB}

All raw data were analyzed using MATLAB 2012b (Mathworks Inc., Natick, MA) scripts. The

PA RF signal was stored as in-phase (I) and quadrature out of phase (Q) baseband signal (IQ 
modulated form). Because the sampling was done at 2x quadrature mode the usable bandwidth of the signal was twice the carrier frequency, $64 \mathrm{MHz}$ (this corresponds to a $128 \mathrm{MHz}$ Nyquist sampling frequency). The I and Q data were interpolated by a factor of 16 and shifted to the carrier frequency $(32 \mathrm{MHz}$ ) to obtain the demodulated pre beamformed PA RF signal. The pre beamformed data was then beamformed using Matlab script provided by Visualsonics to reconstruct PA RF signal.

The US data was already beamformed in the VEVO 2100 hardware and stored as IQ data. US signal was reconstructed by the same process of IQ demodulation as for PA signals. All subsequent processing were applied to these beamformed PA and US data.

\subsubsection{Alignment}

Care was taken in placing the phantom as horizontally level as possible. Ensuring the blood vessel was at same depth on both the left and right side of the B mode image was not possible at the mm scale. Alignment was done in MATLAB where the beamformed PA RF lines were aligned by using a peak detection technique. The result was a horizontally aligned vessel. The alignment process facilitated the use of a rectangular ROI to select subset of the PA signal from different regions of the vessel.

\subsubsection{Signal ROI and SNR}

Due to the complex spatial distribution of RBCs for the different levels of HCT and aggregation, the signal from the vessel was sectioned into three regions: the proximal edge of the vessel, the middle of the vessel and the distal edge of the vessel. Each of these sections was $0.3 \mathrm{~mm}$ long axially to prevent any overlap between segments. The US signal envelope was used to determine 
the geometrical start and end of the vessel in each phantom. The peak receiving sensitivity for the transducer was at $25 \mathrm{MHz}$ therefore the signal sections were each about $5 \lambda$ in length.

Lateral cross range of $1.1 \mathrm{~mm}$ was chosen from the middle of the B mode images. There were 20 A lines (each spatially spaced by $55 \mu \mathrm{m}$ ) in this lateral range of $1.1 \mathrm{~mm}$ across the phantom. Starting lateral distances are shown in Table 2-3.

\begin{tabular}{ccc}
\hline $\begin{array}{c}\text { Experiment 1 } \\
\text { Start (mm) }\end{array}$ & $\begin{array}{c}\text { Experiment 2 } \\
\text { Start (mm) }\end{array}$ & $\begin{array}{c}\text { Experiment 2 } \\
\text { Start (mm) }\end{array}$ \\
\hline 6.27 & 6.11 & 6.22 \\
7.65 & 3.80 & 6.27 \\
5.12 & 5.45 & 5.34 \\
5.28 & 3.74 & 6.71 \\
4.79 & 6.33 & 5.06 \\
5.39 & 5.72 & 8.47 \\
\hline
\end{tabular}

Table 2-3: Lateral distance for experiments chosen for analysis.

Noise was estimated by selecting an ROI (with same dimensions as the signal ROI) from the region between the transducer and phantom that contained PBS. Thus the use of same number of A lines and sample numbers from noise and signal RF data were ensured for the SNR calculation. SNR was calculated by taking the ratio of the root mean square of the envelope of the beamformed noise and signal RF data. In MATLAB the following functions were used: rms(abs(hilbert(signal)))./ rms(abs(hilbert(noise))). 


\subsubsection{Signal window}

Each of the signal sections were multiplied by a hamming window of same length ( $5 \lambda$ in length) to minimize spectral leakage (distribution of the energy of the signal power spectrum to surrounding frequencies).

\subsubsection{Fourier transform and averaging}

The windowed time domain signal was Fourier transformed in MATLAB using the Fast Fourier Transform (FFT) algorithm. The transformation was done using number of points given by $4^{\wedge}$ nextpow2(N), where $N$ was the length of the time domain signal. nextpow2(N) gives a number that can be expressed as an integer power of 2 which is immediately larger than $N$. This ensured the FFT algorithm ran faster since number of transformation points had a base of 2. Choosing $4^{\wedge}$ nextpow $2(N)$ points prevented aliasing as it is much larger than $N$. The absolute value of the transformed signal $Z$ was taken using the $a b s(Z)$ function in MATLAB and then log compressed using the 20. $* \log 10(Z)$ function to produce the power spectrum in $\mathrm{dB}$. Each of the 20 power spectrum (obtained from 20 spatially separate A lines) was averaged over 20 frames. And then normalization and regression analysis was performed on the 20 power spectrum.

\subsubsection{Normalization}

The gold film spectral response shown in Figure 2-4 was obtained by the same FFT procedure as explained in section 2.3.5.4. A total of 150 A lines with range of $0.3 \mathrm{~mm}$ centered on the gold film were transformed and their absolutes values were then log compressed and averaged. This method of normalization was based on use of planar reflector to normalize signal in US [51]. 
The resulting gold film power spectrum was subtracted from the phantom signals to remove the system dependent response and extract features from the RBCs only.

\subsubsection{Midband fit, spectral slope and signal amplitude}

The PA SA was calculated by measuring the peak to peak values of the RF lines. A linear regression was performed on the normalized power spectrum over the $-6 \mathrm{~dB}$ bandwidth of the transducer (10-48 MHz). Slope of the linear fit to the power spectrum was the SS. The MBF was calculated as the y-value of the fit at $29 \mathrm{MHz}$. For each phantom a total of $20 \mathrm{SS}$ and MBF were obtained (from 20 power spectra). Mean and standard deviation of the 20 values of each spectral parameter were calculated and are presented in the results section.

\subsection{Statistical analysis}

All data were analyzed in the Prism 6 statistical software (Graph Pad Software, Inc.). Data normality was tested by performing a Shapiro-Wilk test with $W>0.05$. Unpaired two tailed t-test was performed on NA and AG sample data with $p$ value of 0.05 or less to establish statistical significance. 


\section{Chapter 3 : Results}

\subsection{Hemocytometer microscopic images}

Figure 3-1 shows representative greyscale NA and AG images obtained from the cell suspension in the hemocytometer. The right image is from NA RBCs and image on left contains mixture of single cells and rouleaux (collectively referred to as units) characteristic of aggregation induced by $3 \%$ DEX-70. In the AG case more pixels represented background. For NA case only single cells resting on their flat surface were seen and fewer pixels were representing the background.

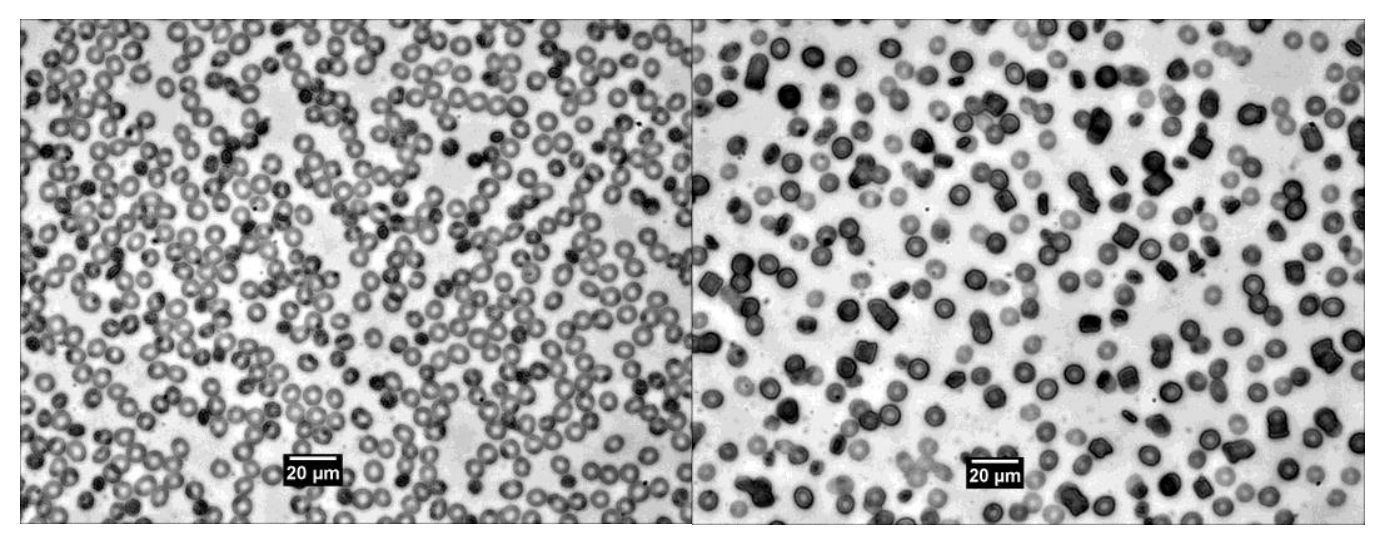

Figure 3-1: Microscopic images of (left) NA washed RBCs in 0\% DEX-70 (right) AG washed RBCs in 3\% DEX-70 at $1 \% \mathrm{HCT}$. 


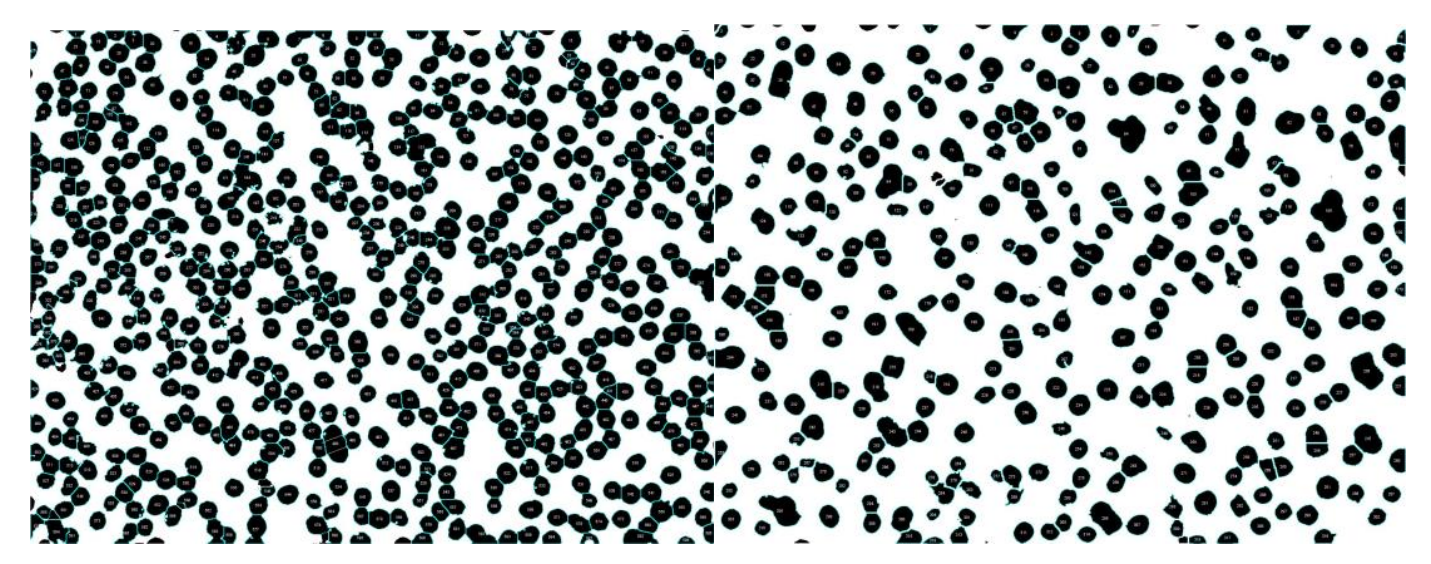

Figure 3-2: Thresholded segmented images after particle analysis (left) NA sample, and (right) AG sample.

Figure 3-2 shows the thresholded images from the NA and AG samples after segmentation and particle analysis.

Figure 3-3 shows the cumulative size distribution for the units in NA and AG samples as a function of area. For NA sample there was no area above $140 \mu \mathrm{m}^{2}$ detected. For the AG samples, units with an area up to $250 \mu \mathrm{m}^{2}$ were measured. The NA sample had $95 \%$ of the units with an area of $70 \mu \mathrm{m}^{2}$ or less and the AG samples had $95 \%$ of the units with an area of $130 \mu \mathrm{m}^{2}$ or less. 


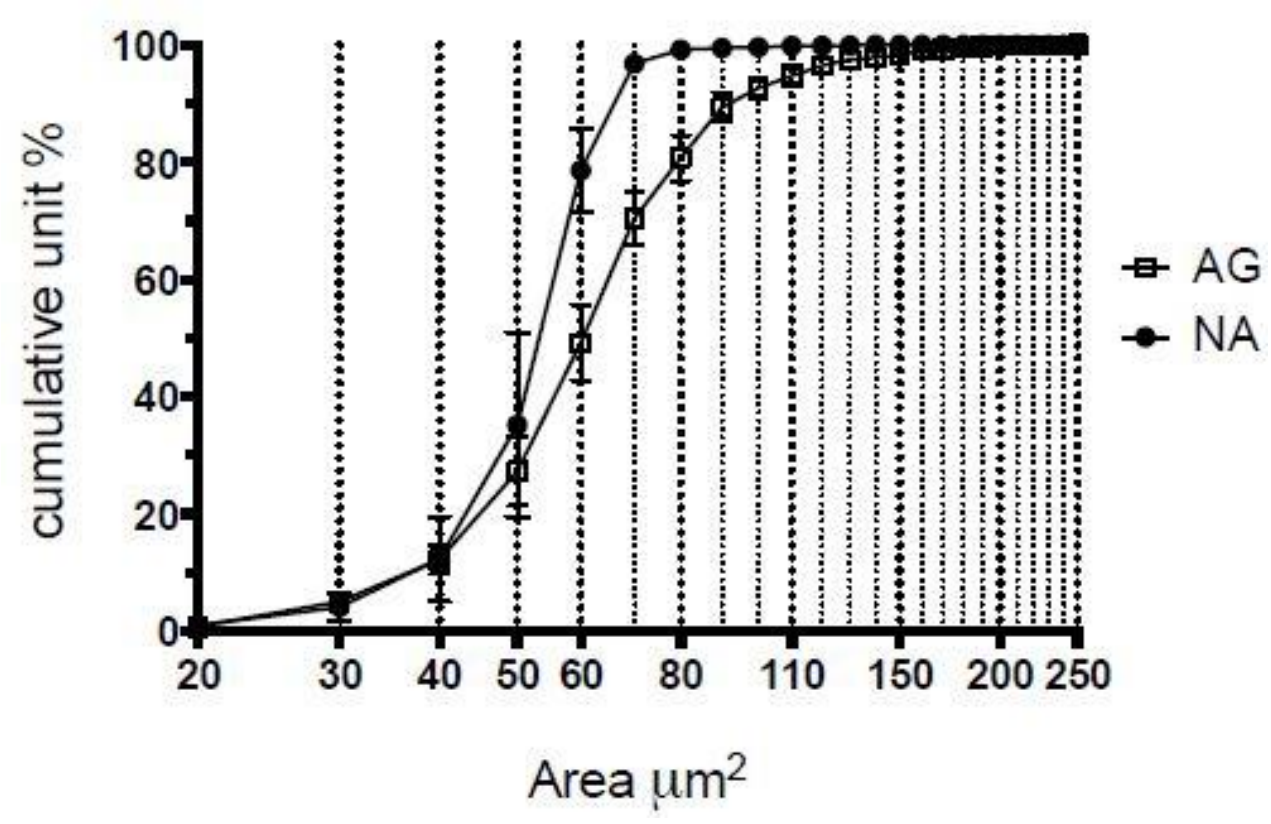

Figure 3-3: Size distribution in $\mu \mathrm{m}^{2}$ for RBC units in the $0 \%$ DEX-70 sample (NA) and for washed RBC units in $3 \%$ DEX-70 solution (AG) . Each unit is n number of RBCs attached together, where $n$ was seen to vary experimentally from 1 to 13 .

The area of a typical single RBC on its flat size was about $60 \mu \mathrm{m}^{2}$. Sample rouleau and single cells on their edges selected for edge area projection are shown in Figure 3-4 and their intensity profiles along the axial lines are shown in Figure 3-5.

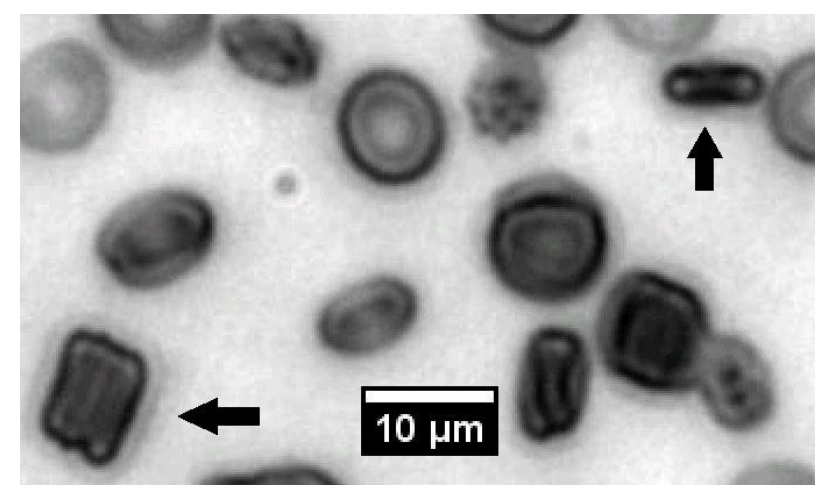

Figure 3-4: Zoomed in image of an AG sample. The arrows point to the units selected for projected RBC side area calculation. 

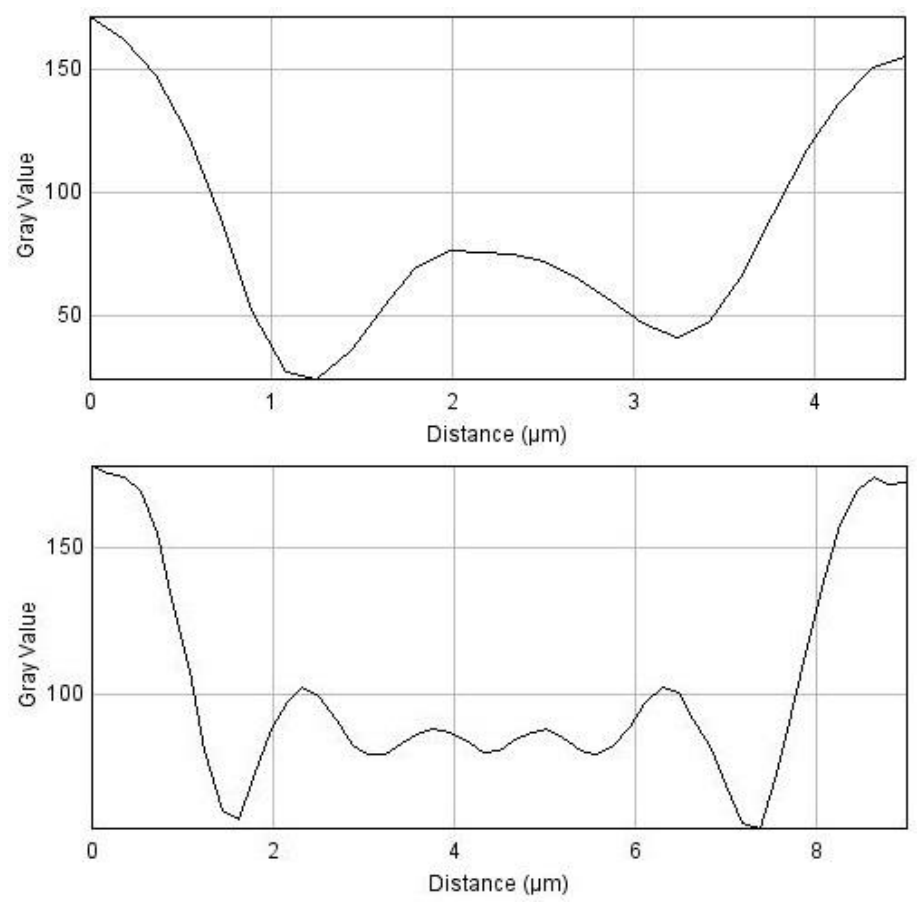

Figure 3-5: (Top) axial intensity profile for typical single RBC on its side and (bottom) axial intensity profile for a typical rouleaux. The width of single cell is about $2 \mu \mathrm{m}$ and the width of the rouleaux is about $6 \mu \mathrm{m}$. For the rouleau the dips indicate aggregation of four RBCs in this unit.

The dips in the intensity in Figure 3-5 (top) indicate there was one RBC in the unit and (bottom) four RBCs in the unit. An average projected area of RBC on its side was determined to be 15 $\mu \mathrm{m}^{2}$. 


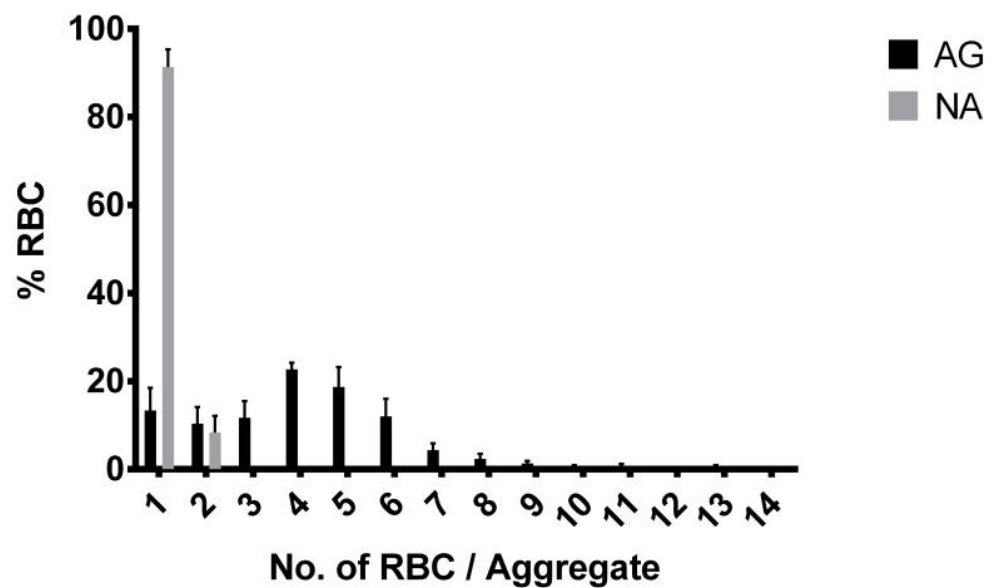

Figure 3-6: Percentage of RBCs in units with incremental number of RBCs per unit. The results for NA sample show about $90 \%$ of the cells are single cells. Most of the units in the AG samples contained four cells.

Figure 3-6 shows the histogram of the number of RBCs per unit measured for NA and AG samples. The numbers represent the mean of measurement for samples from three different days and the error bars represent the standard deviation. For the NA samples $90 \%$ of cells were contained in units of one (single cells) and 10\% in units of two cells. For the AG samples, most cells were contained in units of four cells (rouleaux). And about $80 \%$ of cells were included in units containing 2 or more cells. The maximum number of cells in a unit for AG samples was 13 . 


\subsection{PA B-mode images for $850 \mathrm{~nm}$}
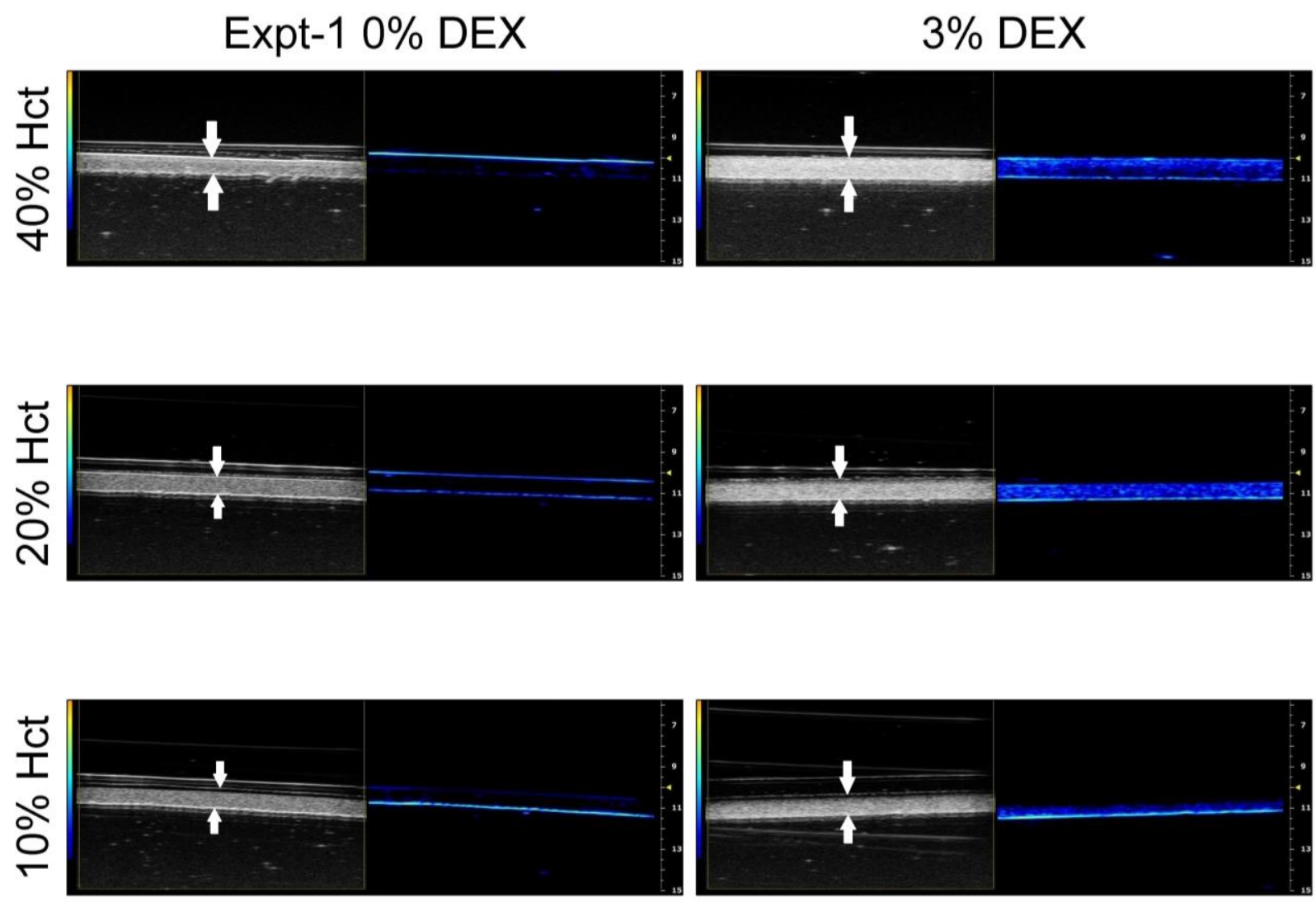

Figure 3-7: Co-reregistered US and PA images as seen on VEVO 2100 screen, on the left greyscale images represent US B mode images and on the right the blue and black images represent PA B mode images. HCTs of 10, 20 and $40 \%$ suspended in 0\% DEX-70 and 3\% DEX-70 are shown (white arrows indicate the proximal and distal edges of the vessels).

The screenshot from the VEVO 2100 LAZR is shown in Figure 3-7. The grayscale images are

US B mode images, and colored images on the right are PA B mode images. 

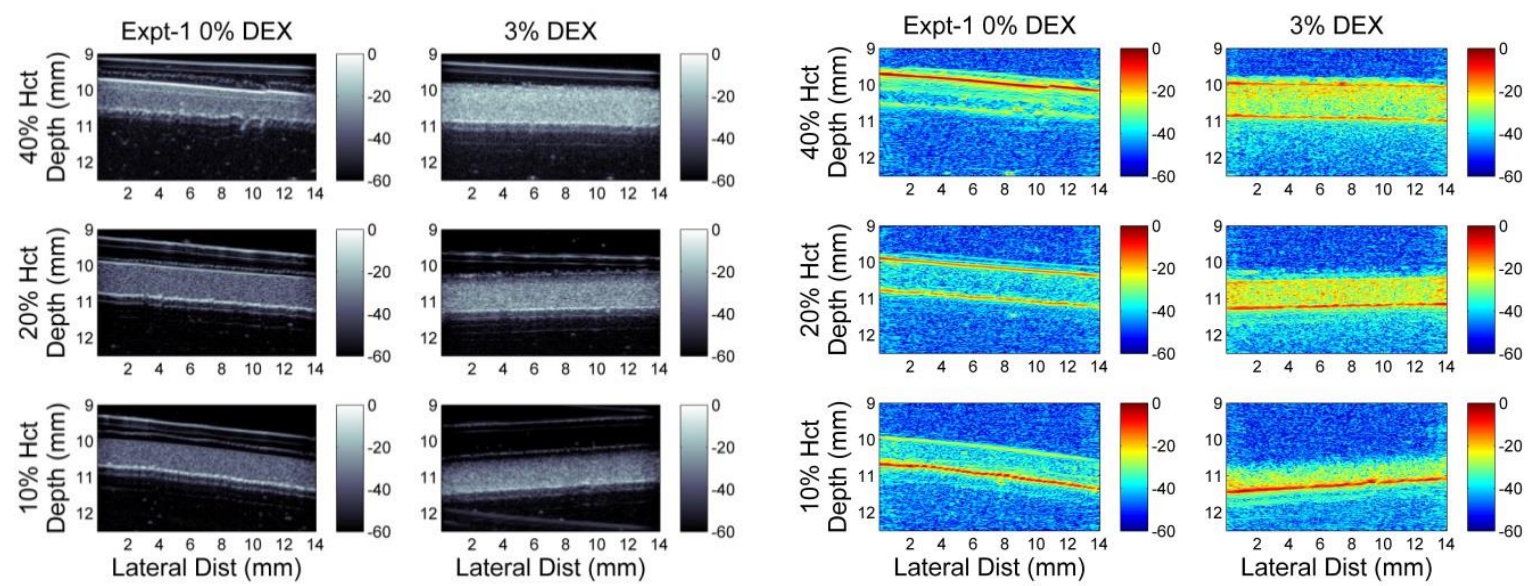

Figure 3-8: Reconstructed US B mode image (left) and PA B mode image (right). B mode image from experiment 1 is shown here. The intensity values are measured in $\mathrm{dB}$.

B mode images reconstructed offline using MATLAB are shown in Figure 3-8. Images on the left are the US reconstructed images and on the right are the PA beamformed images.
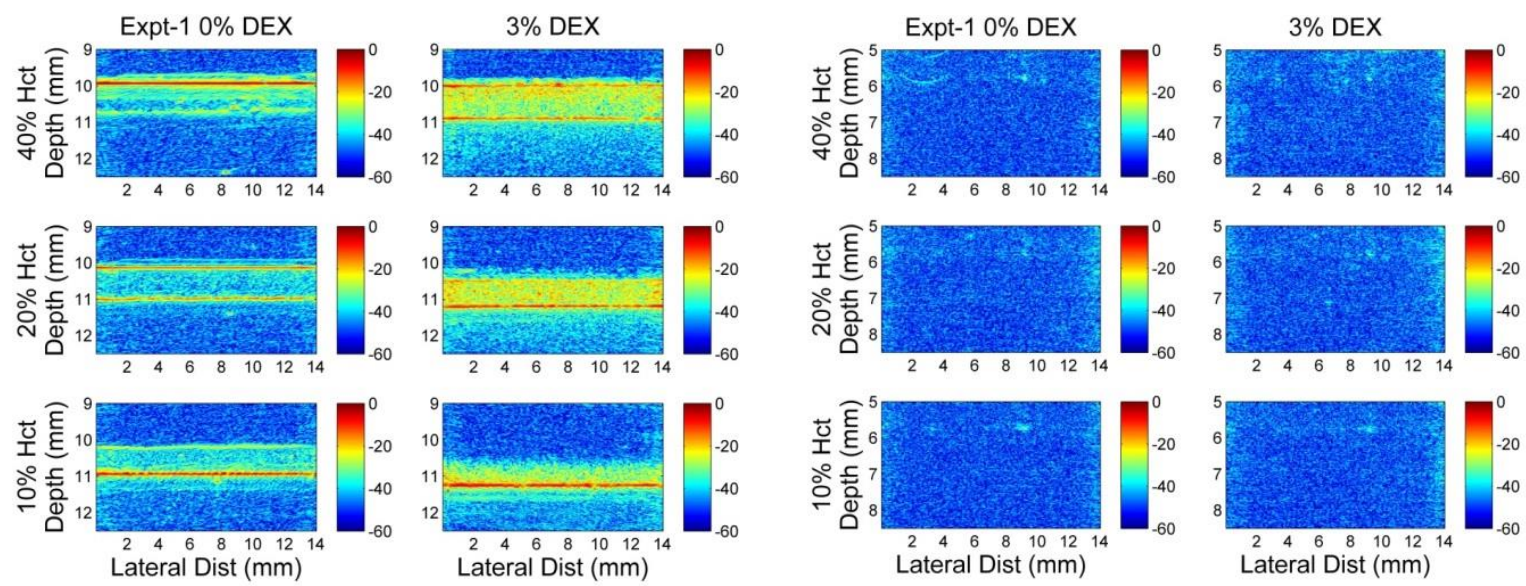

Figure 3-9: PA ROI with vessel realigned (left) and noise from a region between the transducer and phantom (right). $\mathrm{B}$ mode image from experiment 1 is shown here. The intensity values are measured in $\mathrm{dB}$.

Figure 3-9 (left) shows the PA images from Figure 3-8 (right) after the vessel edges have been realigned. Shown on the right are the noise B mode images obtained from a region between the transducer and the phantom. 


\subsection{Time domain signals $850 \mathrm{~nm}$}
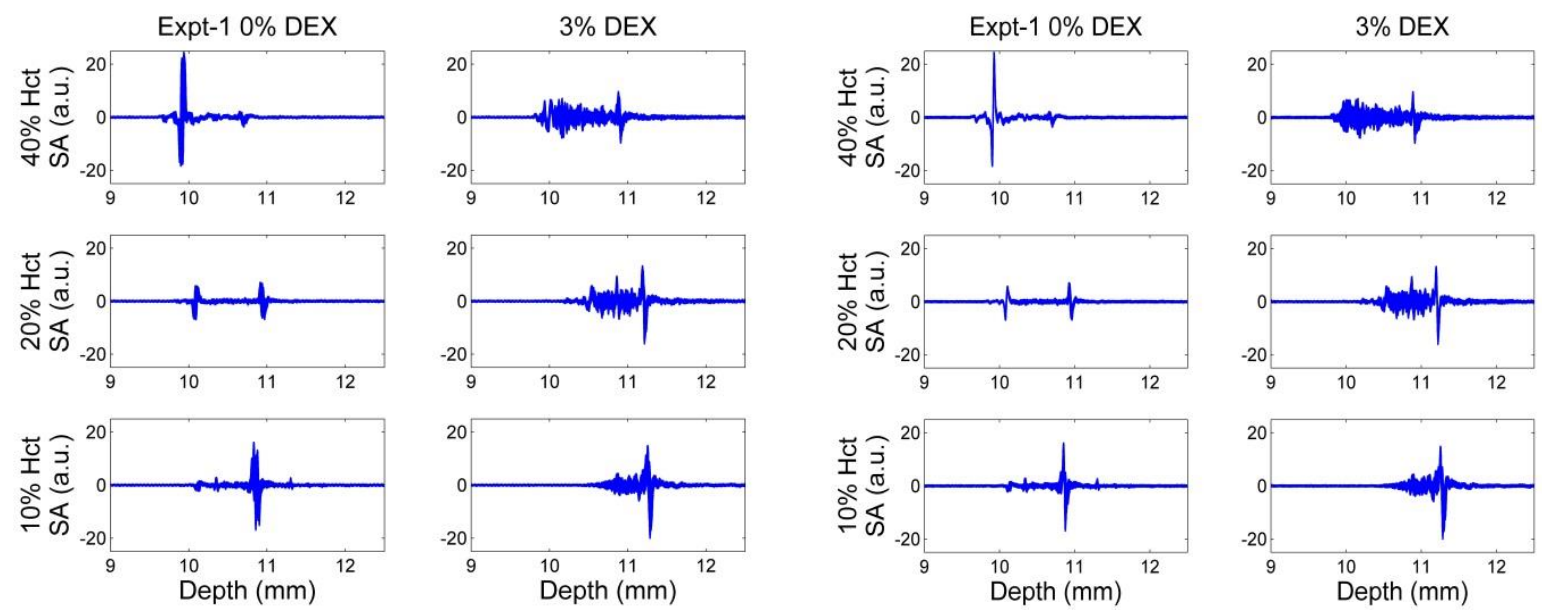

Figure 3-10: 20 A lines averaged over 20 frames were chosen per HCT per DEX-70 concentration for data analysis. (Left) unaligned A lines and (right) aligned A lines.

Time domain PA signals before alignment and after alignment are shown in Figure 3-10 (left) and (right) respectively. Figure 3-11 shows the envelope of the time domain signals from both PA and US modes.
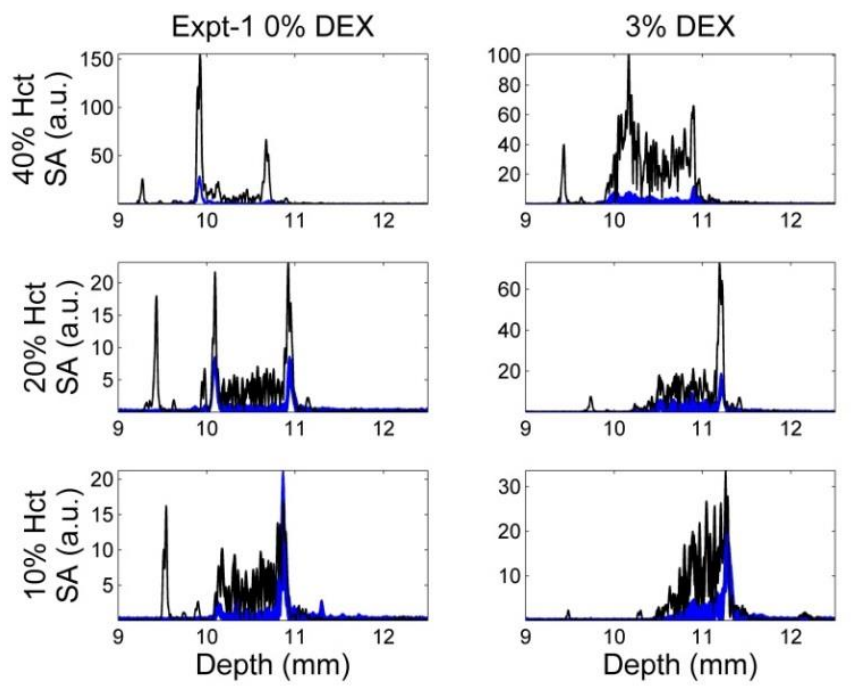

Figure 3-11: Envelopes of US (black) and PA (blue) A lines. US A lines were used to determine the beginning, middle and end of the vessels. Data from experiment 1 is shown in this figure. 

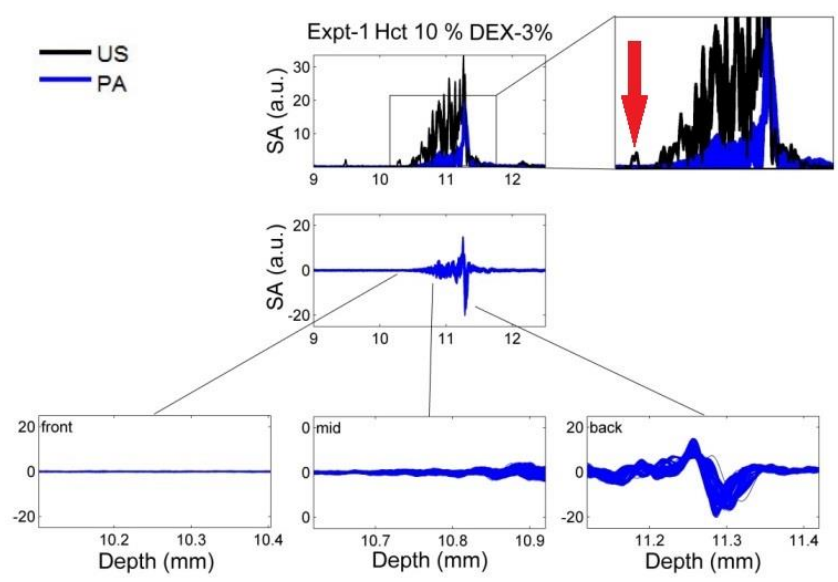

Figure 3-12: (Inset) a zoomed image to show the start (red arrow) of the vessel as indicated by the US envelope, due to settling there is no corresponding PA signal at the vessel start. The sectioned A lines obtained by using the US measurements are shown in the bottom row.

An illustration of how the signal was segmented into its proximal edge, middle and distal edge is shown in Figure 3-12 the top inset shows a typical example of the US envelope that was used to determine the start of the vessel. The first peak at about $10.2 \mathrm{~mm}$ mark obtained from the US envelope (red arrow) was the start of the vessel and the last peak of the envelope was at about $11.3 \mathrm{~mm}$ mark. The middle of the vessel was defined as the halfway distance between the two ends. The signals were segmented with $0.15 \mathrm{~mm}$ on either side of the proximal peak, middle point and distal peak.

\subsection{Signals obtained from the proximal edge of the vessels at $850 \mathrm{~nm}$}

\subsubsection{Time domain signals}

Figure 3-13 shows one sample representative signal for NA and AG RBCs for $850 \mathrm{~nm}$. Data is shown from the $20^{\text {th }}$ frame for experiment 1,2 and 3. Signals were obtained from the proximal edge of the vessels for $40 \%$ HCT. 

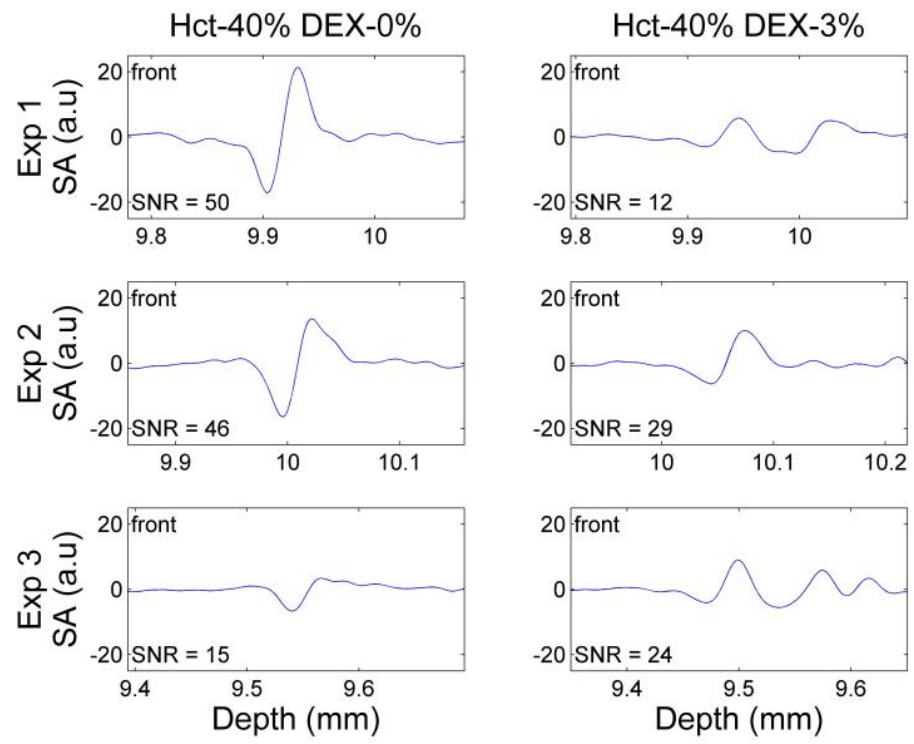

Figure 3-13: Representative A line signals from the proximal edge of the vessels for $40 \%$ HCT obtained at $850 \mathrm{~nm}$ illumination. Left column is from NA samples and right column from AG samples. Exp1, exp 2 and exp 3 represent results from experiment 1,2 and 3 respectively.

Figure 3-14 shows the mean SA at $850 \mathrm{~nm}$ illumination repeated on three days. For $40 \% \mathrm{HCT}$ the SA decreased by 28 (A.U) $(p<0.05)$ in experiment 1 , by 11 (A.U) $(p<0.05)$ in experiment 2 and increased by 6 (A.U) $(p<0.05)$ in experiment 3 . 


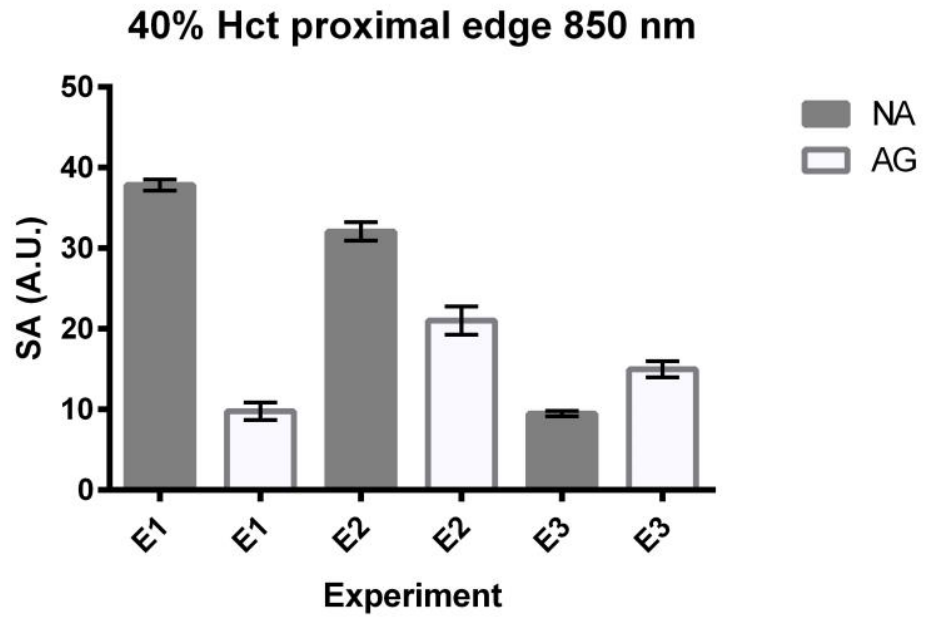

Figure 3-14: Experimental SA at $40 \% \mathrm{HCT}$ for $850 \mathrm{~nm}$ illumination from the proximal edge of the vessels. SA was calculated by obtaining the peak to peak value of the signals. Error bars represent standard deviation of 20 PA RF lines. E1, E2 and E3 mean experiment 1, experiment 2 and experiment 3 respectively.

\subsubsection{Power spectrum}

Figure 3-15 shows the mean power spectrum form the proximal edge of the vessel at $850 \mathrm{~nm}$ repeated on three days, the spectral parameters are extracted from the red linear regression fit lines. In Figure 3-16 the SS decreased by $0.32 \mathrm{~dB} / \mathrm{MHz}(p<0.05)$ in experiment 1 , by 0.20 $\mathrm{dB} / \mathrm{MHz}(p<0.05)$ in experiment 2 and by $0.22 \mathrm{~dB} / \mathrm{MHz}(p<0.05)$ in experiment 3. In Figure 3-17 the MBF decreased by $19 \mathrm{~dB}(p<0.05)$ in experiment 1 , by $5 \mathrm{~dB}(p<0.05)$ in experiment 2 and by $4 \mathrm{~dB}(p<0.05)$ in experiment 3 . 

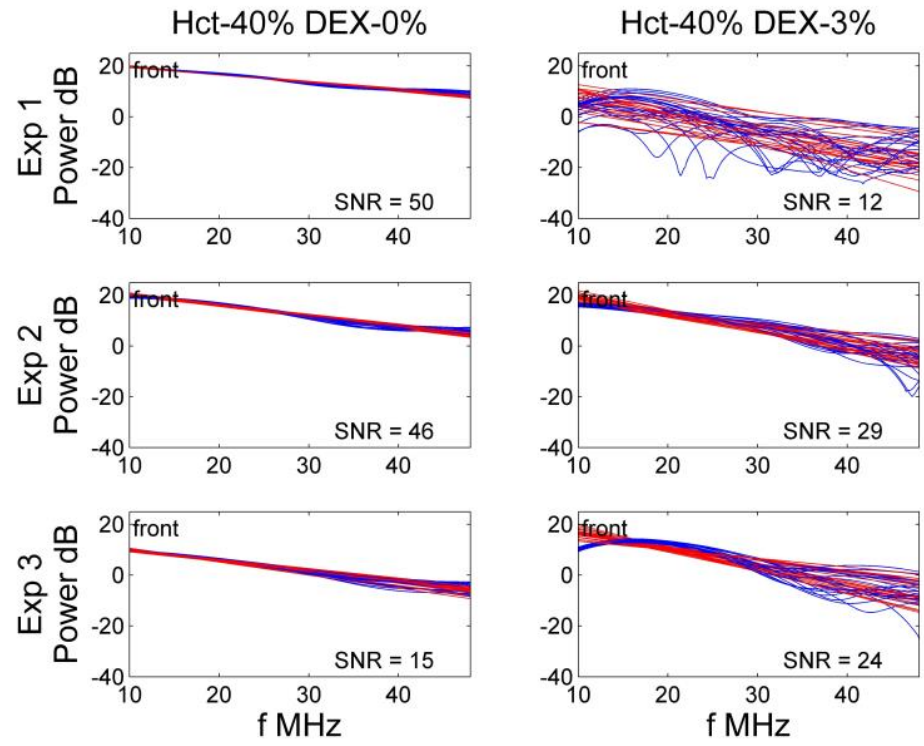

Figure 3-15: Power spectra and linear fit at 40\% HCT for $850 \mathrm{~nm}$ illumination. The left column represents NA samples and the right column represents AG samples. Blue lines are the power spectra and red lines represent the linear fit. In each subplot there are 20 power spectra which were transformed from A lines obtained from 20 different lateral positions in the phantom.

\section{$40 \%$ hct proximal edge $850 \mathrm{~nm}$}

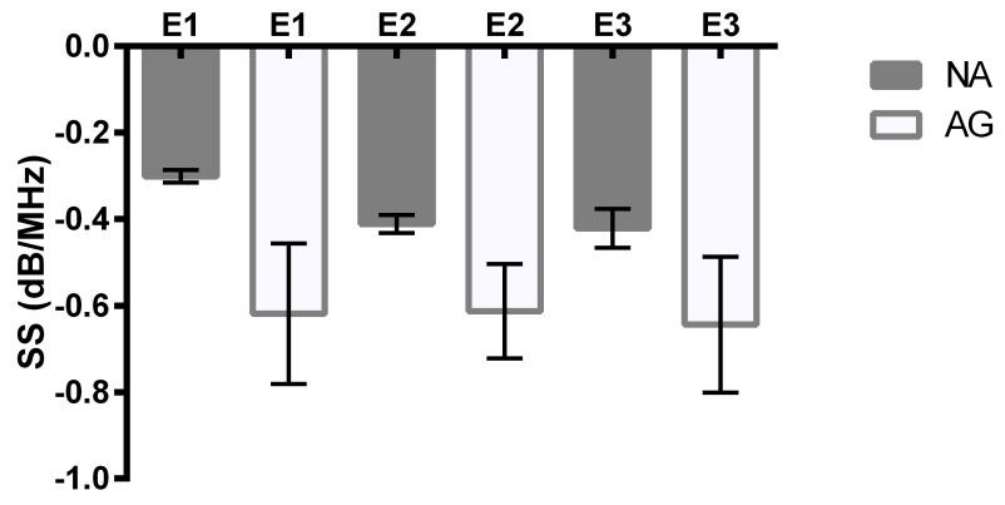

Figure 3-16: Experimental results for SS at $40 \% \mathrm{HCT}$ for $850 \mathrm{~nm}$ illumination from the proximal edge of the vessel. The error bars represent standard deviation of 20 PA spectra. 


\section{$40 \%$ hct proximal edge $850 \mathrm{~nm}$}

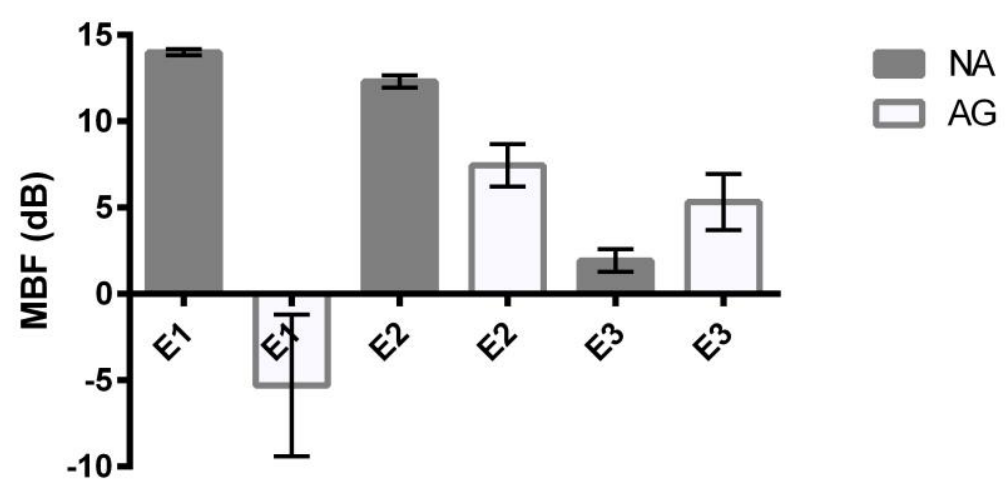

Figure 3-17: Experimental results for MBF at $40 \% \mathrm{HCT}$ for $850 \mathrm{~nm}$ illumination from the proximal edge of the vessel. The error bars represent standard deviation of 20 PA spectra.

\subsection{Signals obtained from the proximal edge of vessels at $750 \mathrm{~nm}$}

\subsubsection{Time domain signal}

Figure 3-18 shows one sample representative signal for NA and AG RBCs for $750 \mathrm{~nm}$. Data is shown from the $20^{\text {th }}$ frame for experiment 1 . Signals were obtained from the proximal edge of the vessels for $40 \% \mathrm{HCT}$. 

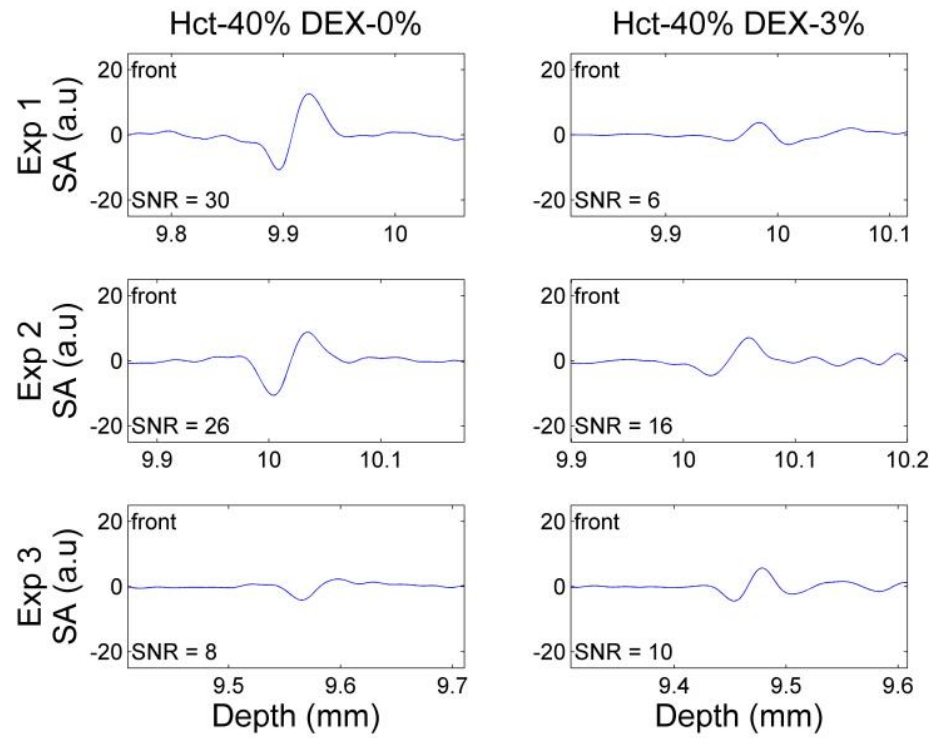

Figure 3-18: Representative A line signals from the proximal edge of the vessels for $40 \%$ HCT obtained at $750 \mathrm{~nm}$ illumination. Left column is from NA samples and right column from AG samples.

Figure 3-19 shows the mean $\mathrm{SA}$ at $750 \mathrm{~nm}$ illumination repeated on three days.

For $40 \%$ HCT the SA decreased by 16 (A.U) $(p<0.05)$ in experiment 1 , by 4 (A.U) $(p<0.05)$ in experiment 2 and increased by 1 (A.U) $(p<0.05)$ in experiment 3 .

\section{0\% Hct proximal edge $750 \mathrm{~nm}$}

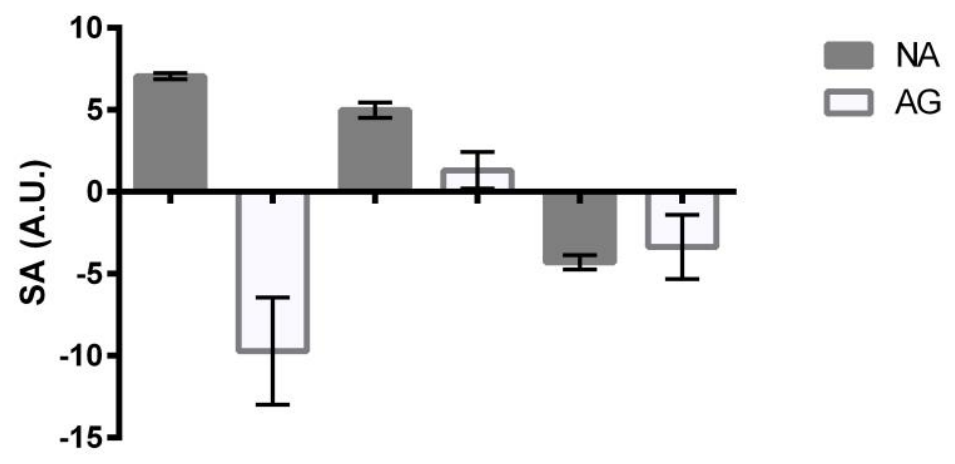

Figure 3-19: Experimental SA at 40\% HCT for $750 \mathrm{~nm}$ illumination. SA was calculated by obtaining the peak to peak value of the signals. Error bars represent standard deviation of 20 PA RF lines. 


\subsubsection{Power spectrum}

Figure 3-20 shows the mean power spectrum form the proximal edge of the vessel at $750 \mathrm{~nm}$ repeated on three days. In Figure 3-21 for 40\% HCT the SS decreased by $0.11 \mathrm{~dB} / \mathrm{MHz}(p<0.05)$ in experiment 1 , by $0.19 \mathrm{~dB} / \mathrm{MHz}(p<0.05)$ in experiment 2 and by $0.21 \mathrm{~dB} / \mathrm{MHz}(p<0.05)$ in experiment 3. In Figure 3-22 for 40\% HCT the MBF decreased by $17 \mathrm{~dB}(p<0.05)$ in experiment 1 , by $4 \mathrm{~dB}(p<0.05)$ in experiment 2 and by $2 \mathrm{~dB}$ (not statistically significant) in experiment 3 .
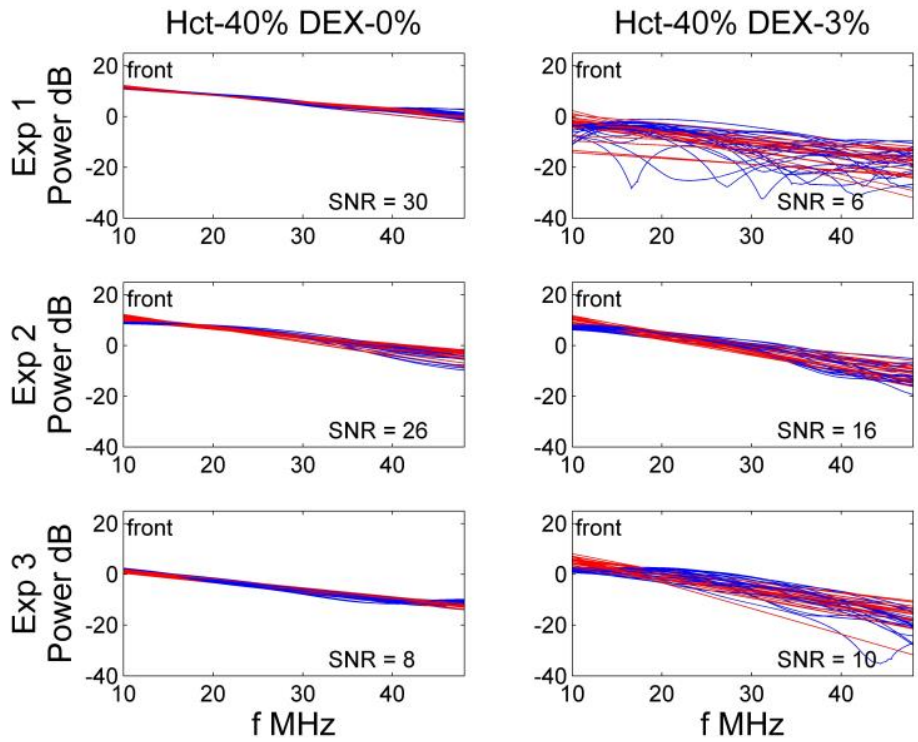

Figure 3-20: Power spectra and linear fit at $40 \%$ HCT for $750 \mathrm{~nm}$ illumination. The left column represents NA samples and the right column represents AG samples. Blue lines are the power spectra and red lines represent the linear fit. In each subplot there are 20 power spectra which were transformed from A lines obtained from 20 different lateral positions in the phantom. 


\section{$40 \%$ hct proximal edge $750 \mathrm{~nm}$}

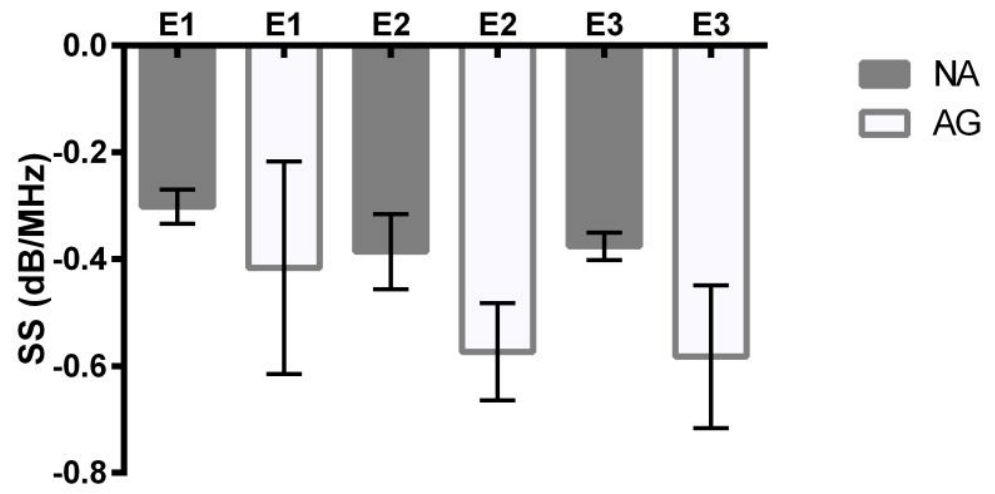

Figure 3-21: Experimental results for SS at $40 \% \mathrm{HCT}$ for $750 \mathrm{~nm}$ illumination from the proximal edge of the vessel. The error bars represent standard deviation of 20 PA spectra.

\section{$40 \%$ hct proximal edge $750 \mathrm{~nm}$}

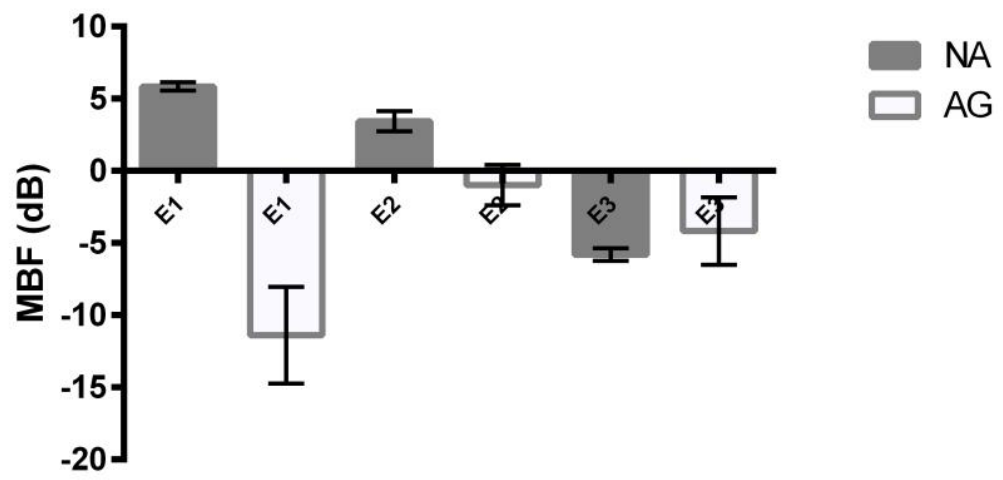

Figure 3-22: Experimental results for MBF at $40 \% \mathrm{HCT}$ for $750 \mathrm{~nm}$ illumination, from the signal at the proximal edge of the vessels. The error bars represent standard deviation of 20 PA spectra. 


\subsection{Oxygen saturation determination from the proximal edge signals}

Using Equation (1-2) $\mathrm{RBC} \mathrm{SO}_{2}$ is calculated and shown in Figure 3-23. Results show high $\mathrm{SO}_{2}$

levels for both NA and AG RBC. In Figure 3-23 the $\mathrm{SO}_{2}$ increased by $10 \%(p<0.05)$ in experiment 1 , by $5 \%(p<0.05)$ in experiment 2 and by $8 \%(p<0.05)$ in experiment 3 .

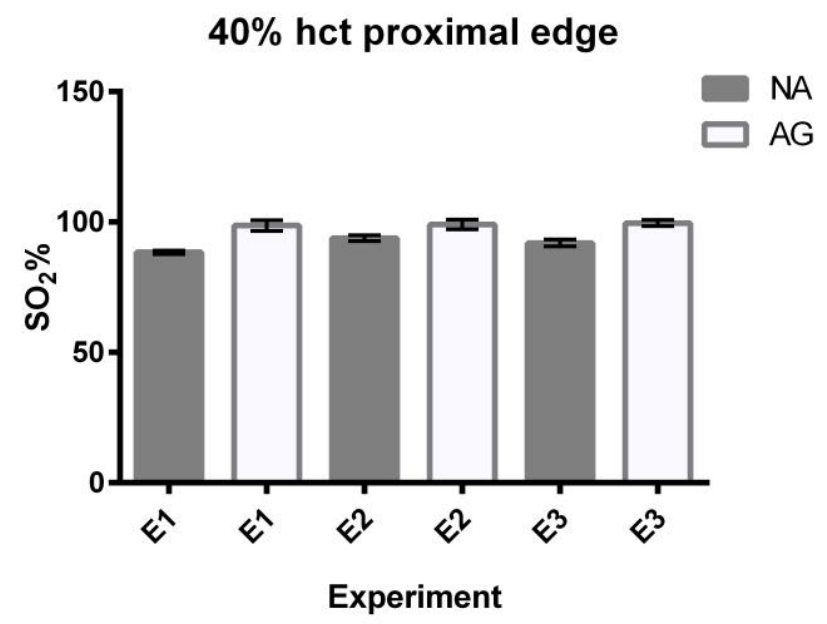

Figure 3-23: $\mathrm{SO}_{2}$ for AG samples for the three experiments. $\mathrm{SO}_{2}$ was calculated by using the $\mathrm{SA}$ from the proximal edge of the vessels for the $40 \%$ HCT samples.

\subsection{Signals obtained from the middle section $850 \mathrm{~nm}$}

\subsubsection{Time domain signal}

Figure 3-24 shows one sample representative signal (out of 20 total) for NA (0\% DEX-70) and AG RBCs (3\% DEX-70) data is from the $20^{\text {th }}$ frame for experiment 1 . 

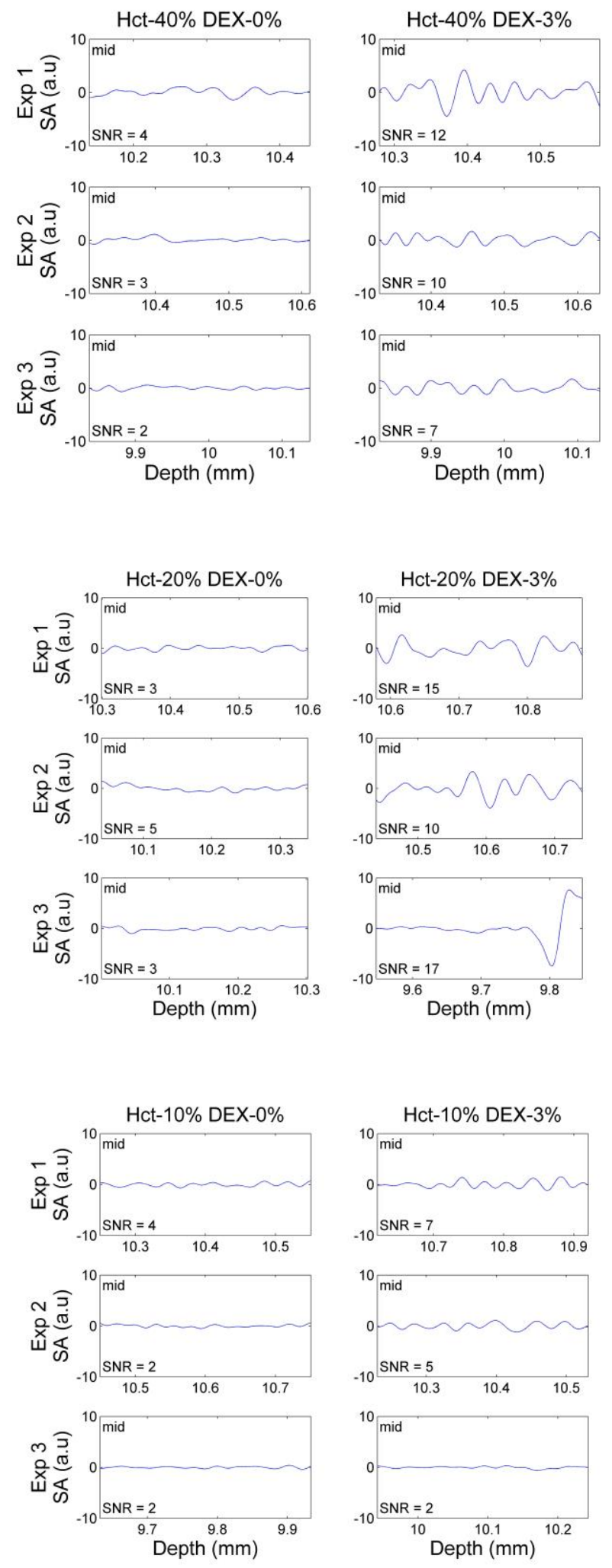

Figure 3-24: Representative A line signals from the middle section of the vessels (top) $40 \%$ HCT, (middle) $20 \%$ HCT and (bottom) 10\% HCT for $850 \mathrm{~nm}$ illumination. Left column is from NA samples and right column from AG samples. 
Figure 3-25 shows the mean SA form the middle section of the vessel obtained by averaging 20 frames per A line and 20 A lines per phantom. Results from three experiments repeated on three days are shown. The transducer required a setup procedure which introduced a time delay of anywhere from 1 to 5 minutes between transducer positioning and image acquisition. This allowed for some RBC sedimentation to occur between the times the phantom was setup and actual imaging could begin. As sedimentation was an uncontrolled factor in each measurement, data from the repeat experiments are presented separately.

Figure 3-25 shows for 40\% HCT the SA increased from NA to AG by 8 (A.U) $(p<0.05)$ in experiment 1 , by 6 (A.U) $(p<0.05)$ in experiment 2 and by $5($ A.U) $(p<0.05)$ in experiment 3. For $20 \%$ HCT the SA increased by 12 (A.U) $(p<0.05)$ in experiment 1 , by 6 ((A.U) $(p<0.05)$ in experiment 2 and by 15 (A.U) $(p<0.05)$ in experiment 3. For $10 \%$ HCT the SA increased by 3 (A.U) $(p<0.05)$ in experiment 1 , by 3 (A.U) $(p<0.05)$ in experiment 2 and by 0.28 (A.U) $(p<0.05)$ in experiment 3. The SNR (value of 2) was uncharacteristically low for the $10 \%$ HCT AG sample in experiment 3. This particular data point is marked by a square and triangle in Figure 3-25 and the anomaly commented on in the discussion. 

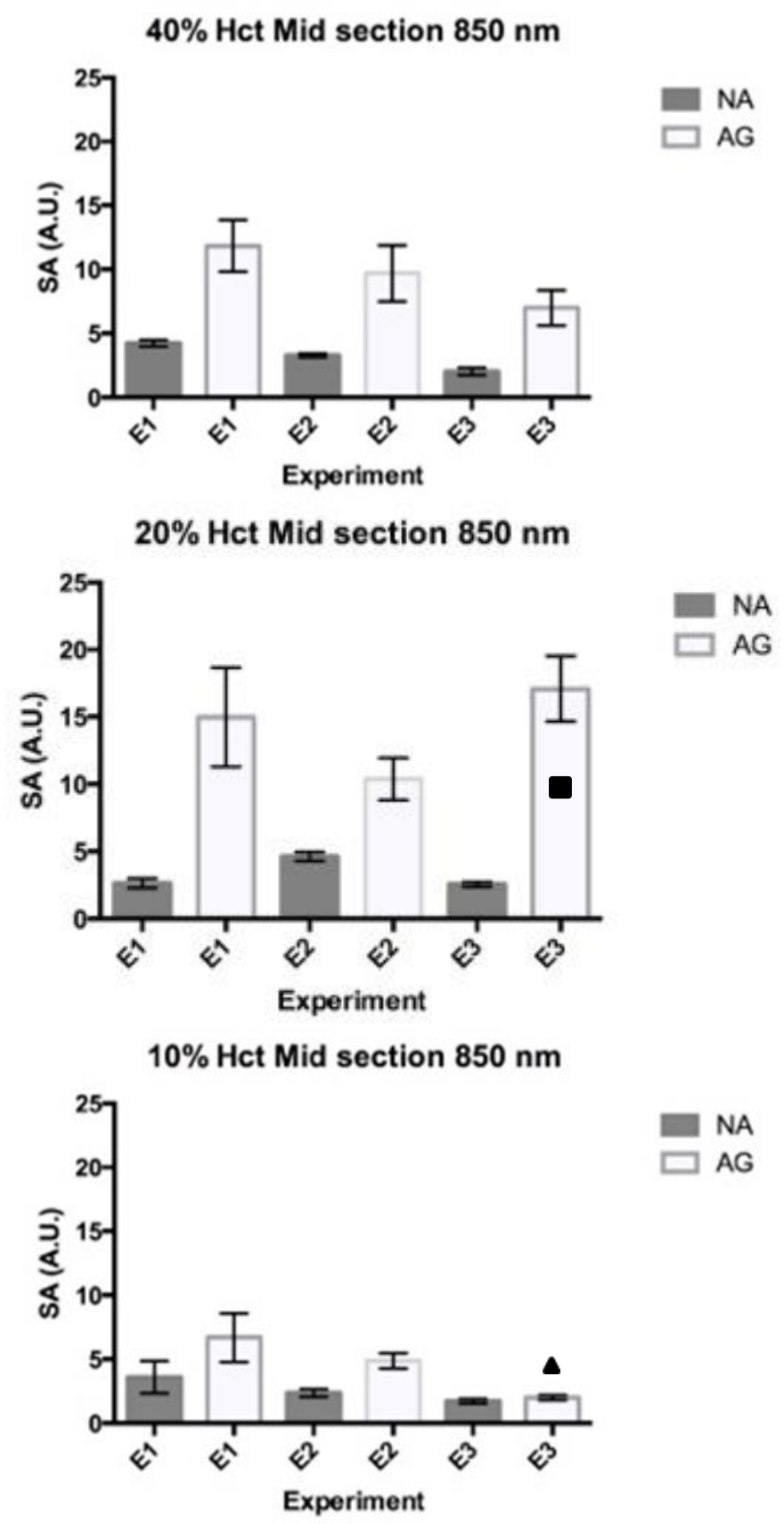

Figure 3-25: Experimental SA (top) 40\% HCT, (middle) 20\% HCT and (bottom) 10\% HCT for $850 \mathrm{~nm}$ illumination. SA was calculated by obtaining the peak to peak value of the signals.. Error bars represent standard deviation of 20 PA RF lines. 


\subsubsection{Power spectrum}

Figure 3-26 shows 20 power spectra and the corresponding linear regression obtained from the middle section of the vessels. Signals from the left column are from NA RBCs and on right are from AG RBCs.

Figure 3-27 shows the MBF from the middle section of the vessel. For 40\% HCT the MBF increases from NA to AG by $13 \mathrm{~dB}(p<0.05)$ in experiment 1 , by $10 \mathrm{~dB}(p<0.05)$ in experiment 2 and by $11 \mathrm{~dB}(p<0.05)$ in experiment 3 . For $20 \%$ HCT the MBF changes by $+15 \mathrm{~dB}(p<0.05)$ in experiment 1 , by $+13 \mathrm{~dB}(p<0.05)$ in experiment 2 and by $+5 \mathrm{~dB}(p<0.05)$ in experiment 3 . For $10 \%$ HCT the MBF changes by $+4 \mathrm{~dB}(p<0.05)$ in experiment 1 , by $+8 \mathrm{~dB}(p<0.05)$ in experiment 2 and $-1 \mathrm{~dB}(p=0.12$, not statistically significant) in experiment 3. Data points marked with square and triangle in Figure 3-27 are further commented on in the discussion section. 

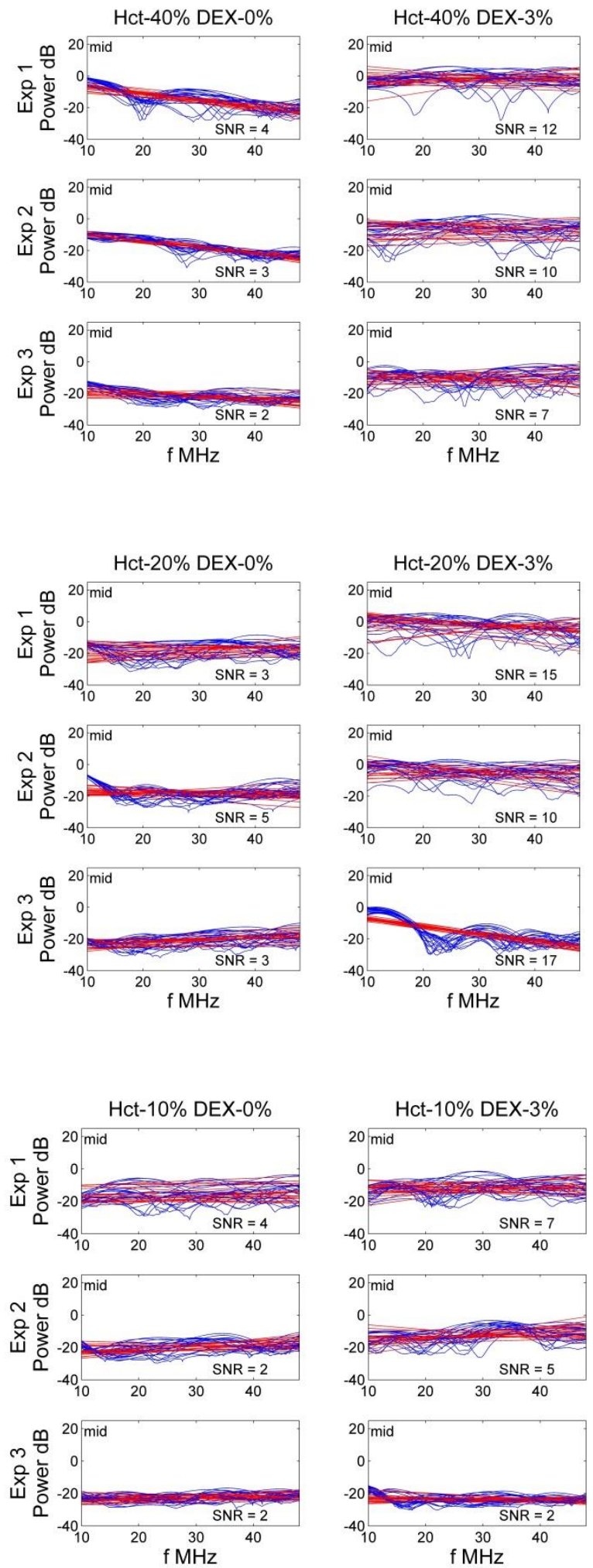

Figure 3-26: Power spectra and linear fit (top) 40\% HCT, (middle) 20\% HCT and (bottom) $10 \%$ HCT for $850 \mathrm{~nm}$ illumination. Left column represents NA samples and right column represents AG samples. Blue lines are the power spectra and red lines represent the linear fit. In each subplot there are 20 power spectra which were transformed from A lines obtained from 20 different lateral positions in the phantom. 
40\% Hct Mid section $850 \mathrm{~nm}$

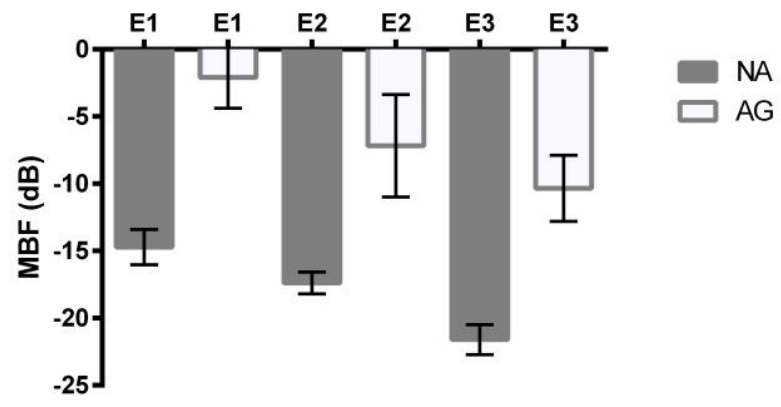

$20 \%$ Hct Mid section $850 \mathrm{~nm}$

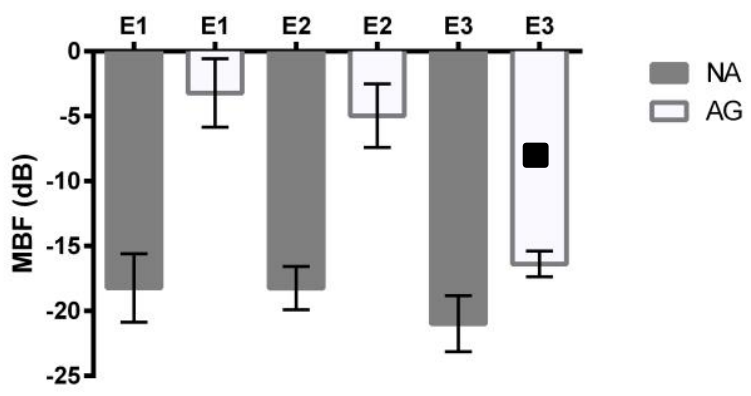

$10 \%$ Hct Mid section $850 \mathrm{~nm}$

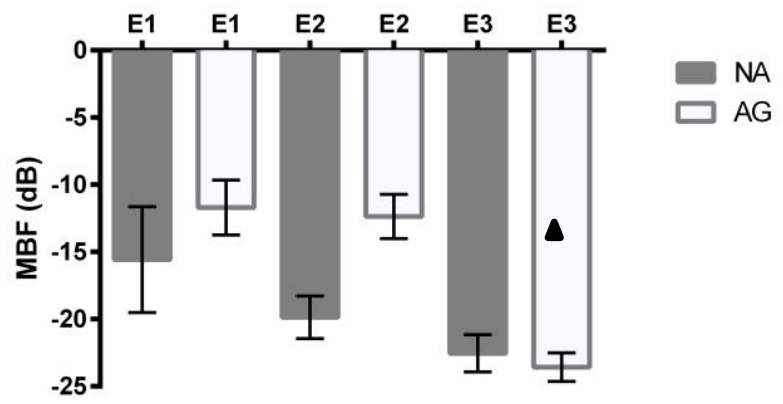

Figure 3-27: Experimental results for MBF (top) 40\% HCT, (middle) 20\% HCT and (bottom) 10\% HCT for $850 \mathrm{~nm}$ illumination. The error bars represent standard deviation of 20 PA spectra. 0\% DEX-70 represents NA samples and $3 \%$ DEX-70 represents AG samples. The square and triangle represent data that suggest fast sedimentation as commented on in the discussion section. 


\section{Chapter 4 : Discussion}

\subsection{Microscopic aggregation quantification}

The optical images in Figure 3-1 show the increased clustering of cells and more clear intercellular space in the AG sample. In Figure 3-3 the cumulative distribution of sizes of aggregates shifts to higher values for the AG sample. The size of the largest area found in the AG case indicates formation of cluster whereas the size of the largest area in the NA case ( 2 cells per unit) may be because of the overlapping of two RBCs that were not correctly watershed.

The results shown in Figure 3-3 are comparable to the findings of a previous study [34] where introduction of DEX lead to larger aggregates. However the sizes of aggregates formed in the previous study were larger in comparison. Several factors including a higher hematocrit and application of low shearing conditions in the previous publication may account for the increased size of aggregates.

The current results for aggregate size can be used to obtain more realistic simulation parameters. The measured distribution of sizes from the experiment can be used to produce a distribution in the simulated sizes of the aggregates. Results in Figure 3-6 are obtained from very low hematocrit (1\%) which limited the formation of large clusters; predominantly rouleaux of certain sizes were observed. Even then it may be scaled to a higher hematocrit, since studies have reported the maximum aggregate size is a function of hematocrit, aggregating agent used, shearing conditions and the size of the vessel. 


\subsection{PA aggregation detection}

\subsubsection{B mode images}

The PA beamformed B mode images for NA and AG samples in Figure 3-8 (right) shows that compared to the NA samples the brightness of the AG samples increased from the middle section of the vessel. This is also seen in the US images Figure 3-8 (left) where aggregation produced brighter areas in the middle of the vessels in comparison to the NA samples. Previous publications [47],[48] reported increase in echogenicity with formation of blood aggregation as shown in Figure 1-7.
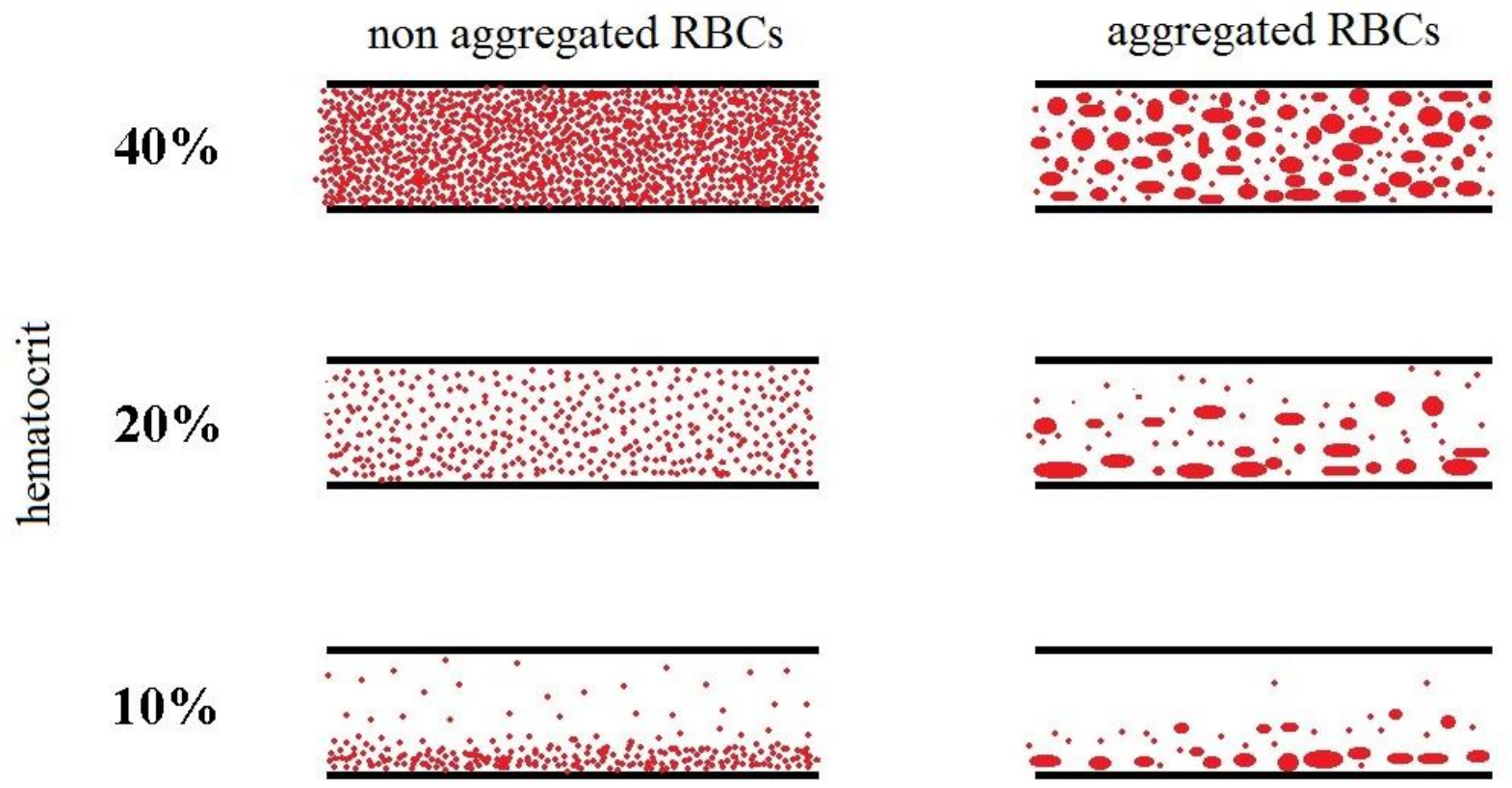

Figure 4-1: Suggested schematic of the distribution of the NA and AG RBCs in the phantom vessels. 
In the US images Figure 3-8 (left) the proximal edge of the vessels can be clearly delineated. For the $20 \%$ and $10 \%$ AG samples the bright echogenic RBC suspension can be seen to be more echogenic at a slightly lower height than the proximal vessel edge. This indicates that the density of RBCs near the proximal vessel edge was less, thus RBCs were settling at a higher rate for lower HCT and increased AG. In PA images Figure 3-8 (right) the increased brightness of the lower edge also indicates increased sedimentation. The results are consistent with the finding of increased settling for lower HCT and higher aggregation reported by Kinnunen [40] .

Figure 4-1 shows the suggested distribution of the RBCs in the phantom vessels. For 40\% HCT the sedimentation is assumed to be least significant for both the NA and AG samples. For 10\% HCT the sedimentation is assumed to be most significant for the NA samples. For $20 \%$ HCT the NA RBCs are assumed to have had some sedimentation but the AG samples may have sedimented to a greater extent. The RBC distribution in Figure 4-1 is thought to represent the state of RBCs in experiment 1 and 2. However for experiment 3 the sedimentation may have been further progressed and possibly no cells were left in the middle section of the vessels for the $20 \%$ and $10 \%$ HCT samples.

\subsubsection{Time domain signals}

The results of the time domain signals are shown in Figure 3-10. Results obtained show the complex interplay of several different factors: changes in optical absorption, scattering and blood sedimentation with aggregation and HCT. The setup time was not same from phantom to phantom which resulted in different amounts of sedimentation in the samples examined in this work. Figure 1-13 shows that increase in HCT results in reduction of sedimentation rate and aggregation accelerates the rate. It is speculated that the variation in the results from experiment 
to experiment can be attributed to the different amount of sedimentation that had occurred before the data acquisition. The lack of control of sedimentation rate in has a greater impact on the results for the $20 \%$ and $10 \%$ AG samples (especially in experiment 3).

In Figure 3-10 for the 40\% HCT NA sample absorption of light was mostly at the proximal edge of the vessel producing a sharply peaked PA SA. The concentration of RBCs makes the suspension highly attenuating and most of the light was absorbed within the first few millimeters (intensity decreases by half at $0.43 \mathrm{~mm}$ for $850 \mathrm{~nm}$ ) [44], which results in very strong signals from the proximal edge, Figure 3-13 and Figure 3-18. This explains why the SA was significantly lower from the middle and proximal edge of the vessel even though RBCs were uniformly distributed throughout the vessel for $40 \%$ HCT NA samples (Figure 3-10). The uniform distribution of RBCs is supported by the uniform speckle throughout the vessel on the B mode images in Figure 3-8 for NA 40\% HCT samples. The AG samples on the other hand did not display the sharp first peak at the proximal edge of the vessels (Figure 3-10, Figure 3-13 and Figure 3-18) since aggregation reduced overall absorption and increased transmission of light through the suspension due to increased plasma gaps. As a result the SA decreases for AG samples in comparison to the NA samples at both wavelengths (Figure 3-14 and Figure 3-19) from the proximal edge. An exception to this was observed in experiment 3 (Figure 3-14 and Figure 3-19) where the NA sample had uncharacteristically low SNR for the NA samples at both wavelengths (15 at $850 \mathrm{~nm}$ and 8 at $750 \mathrm{~nm}$ ). This may have been caused due sedimentation (experiment 3 NA sample with 40\% HCT), which reduced the number of RBCs at the proximal edge, producing a lower SA. These results from the proximal edge suggest that local energy deposition is the main determinant of SA. AG samples have been shown to produce higher SA when compared to NA samples [2], but the results are true only when the local energy deposition 
between the NA and AG samples are not significantly altered due to other compounding factors like sedimentation.

Use of DEX also reduces scattering in AG blood because of RBC and suspending fluid index matching which also aids in increased transmission [90]. In Figure 3-10 for 40\% HCT the combined effect was the optical clearing of blood resulting in the increase in the SA from the middle section of the vessel due to aggregation (Figure 3-24, Figure 3-25). In conjunction the PA SA in the middle also increased with an increase in the effective absorber radius [91]. The distal edge produced low SA due to attenuation of light.

For $20 \%$ HCT the NA samples produced a weaker PA SA from the proximal edge as the number of absorbers was less in comparison to $40 \%$ HCT (Figure 3-10). An increase in optical transmission due to lower HCT at $20 \%$ was evident since the SA of the PA signal from the distal edge was comparable to that of the proximal edge. The PA signal from the proximal edge for the AG samples were not as high as the NA proximal edge signals because aggregation and sedimentation reduced optical energy deposition at the proximal edge. For the NA case the signal from the middle of the vessel comes from single suspended RBCs, where as those from the AG case comes from larger size aggregates. The mean SA (Figure 3-25) calculated from the midsection for all $\mathrm{HCT}$ at $850 \mathrm{~nm}$ illumination presented shows an increase in the SA when RBCs were AG. The increase in absorber size and optical transmission produced a stronger SA from the middle of the vessel (Figure 3-24). In experiment 3 (data point marked by a square in Figure 3-25) the AG sample for $20 \%$ HCT had a significant sedimentation which can be seen by the presence of the RBC-suspension fluid interface in the A line from middle section of the vessels (Figure 3-24). The SA from the distal edge was stronger for AG samples as faster sedimentation increased concentration of absorbers at the distal edge (Figure 3-10). 
For the $10 \%$ HCT samples the sedimentation was the fastest amongst all the other HCTs, and this can be seen from the strong signal from the distal edge of the vessels in Figure 3-10 and increased echogenicity of the distal edge in the US B mode images in Figure 3-8. At 10\% HCT not just the AG sample but also the NA sample produced a stronger SA at the distal edge rather than at the proximal edge. This is speculated to be due to significant sedimentation and greater volumetric concentration at the distal edge. For the NA samples RBCs in the middle section of the vessels had smaller absorber size and hence produced signals with lower SA than that from the larger AG samples (Figure 3-24, Figure 3-25). In experiment 3 the data point marked by a triangle in Figure 3-25 for the 10\% HCT AG sample it was seen that the SA was as low as the NA sample; this was most likely caused due significant sedimentation of the AG sample. Here all the AG RBCs sedimented below the middle section leaving very few absorbers to produce a significant signal. The US image for experiment 3 in appendix Figure A-0-5 for 10\% HCT AG sample also shows a significantly brighter echogenicity at an axial distance lower than the middle section of the vessel, supporting the idea that RBCs have settled beyond the middle section of the vessel. A low SNR of 2 (same as the NA sample) (Figure 3-24) from the midsection of the vessel also supports this conclusion.

With the sedimentation effect explained in experiment 3, the results from the middle section of the vessels are consistent with the low frequency study by Hysi et al [2] that also showed increase in SA with aggregation for both wavelengths. The increase in SA is both due to increased size of the absorbers and the increased optical energy deposition. The impact of aggregation on the SA at the proximal peak was however overpowered by the changes in energy deposition. The changes in optical energy deposition are mainly governed by spatial distribution of the absorbers and the impact the distribution has on the light fluence. Hysi et al [2] also 
reported the effect of HCT on PA SA which showed an increase in SA with increase in but such comparison was not made in this work because sedimentation was an uncontrolled factor, and hence the effect of HCT alone could not be evaluated from these results.

Only results from the proximal peak of the $40 \%$ HCT samples for $750 \mathrm{~nm}$ and $850 \mathrm{~nm}$ were analyzed, since at this HCT the impact of sedimentation was not considered to be significant within the duration of the experimental procedure [40]. Also the overall signal strength form the $750 \mathrm{~nm}$ illumination was lower than the $850 \mathrm{~nm}$ illumination (due to the samples being highly oxygenated) (Figure 3-13, Figure 3-18). This is why all analysis on the signals from the middle of the vessel was confined to $850 \mathrm{~nm}$ only.

\subsubsection{Spectral analysis - SS}

The power spectrum from the proximal edge for the $40 \%$ HCT samples shows very little variation in the NA case (Figure 3-15, Figure 3-20). This is because the RBCs are uniformly distributed and the optical properties do not change from sample to sample. The AG samples show greater variation and some dips in the spectrum (Figure 3-15, Figure 3-20). Especially for experiment 1 AG sample shows a large variation in SS (Figure 3-16, Figure 3-21). This particular data point also has the lowest SNR amongst all the proximal edge signals for both illumination wavelengths. These large variations in the spectral features may be associated with the presence of aggregates because changes in local energy deposition due to non-uniformly sized and randomly distributed aggregates should introduce large variations in PA signals.

Figure 3-16 shows that with aggregation the SS decreases for all three experiments. At $850 \mathrm{~nm}$ illumination the changes are statistically significant for all 3 experiments. For $750 \mathrm{~nm}$ wavelength illumination (Figure 3-21) for experiment 1 the mean SS for AG sample was lower 
than the NA but had large variations, this may be caused by the low SNR value (=6), which may have introduced the large variations in the signal from the AG sample. This demonstrates the need to have a strong SNR to do spectral analysis of PA signals. The results from signals with high SNR matched the expected trend since the PA signal from a larger absorber should produce more low frequency components. Hysi et al [2] also reported the same trend with aggregation where the SS decreases with increase in size.

\subsubsection{Spectral analysis - MBF}

The MBF calculated at $25 \mathrm{MHz}$ from the power spectrum (Figure 3-15 and Figure 3-20) from the proximal edge of the vessels at 850 and $750 \mathrm{~nm}$ indicated that with aggregation the MBF decreased for experiment 1 and 2 (Figure 3-27 and Figure 3-22). For experiment 1 the large change in the mean values of MBF may be instigated by the comparatively lower SNR (12 at $850 \mathrm{~nm}$ and 6 at $750 \mathrm{~nm}$ ) for the AG sample. The low SNR may be due to some sedimentation having occurred for the AG sample. For experiment 3 the NA sample had uncharacteristically low SNR (15 at $850 \mathrm{~nm}$ and 8 at $750 \mathrm{~nm}$ ) amongst all the signals from the NA samples' proximal edges possibly due to sedimentation during the measurement process. This may explain the lower value of the MBF for the NA case. The value of MBF is more susceptible to the changes in the local energy deposit, which is reflected in these results. Increased energy fluence attenuation due to homogeneous presence of RBCs and higher energy deposit at the proximal edge the MBF values are higher for the NA samples. The expected pattern of MBF increasing with increase in size of absorbers [2] was not evident in the signals from the proximal edge of the vessels for the aforementioned reasons. Over all the $\mathrm{MBF}$ for $850 \mathrm{~nm}$ had higher values than the $750 \mathrm{~nm}$ results at $40 \%$ HCT which can be explained by the fact that $\mathrm{HbO}_{2}$ absorbs more at $850 \mathrm{~nm}$ than at 750 nm (Figure 1-1). 
The spectral features for the signals from the middle of the vessels for $850 \mathrm{~nm}$ illumination (Figure 3-26) show very low SNR for the NA samples, as expected due to most of the energy deposit happening at the proximal edge. For the AG samples the SNR was higher due to combined effect of increased optical transmission and increase in aggregate size. For all HCT the MBF increased with aggregation (Figure 3-27), with the following exceptions. The impact of excessive sedimentation (as explained earlier in the time domain signal section) in experiment 3 with 20 and 10\% HCT AG samples are evident in the MBF. The 20\% HCT AG sample in experiment 3 shows less of an increase in MBF compared to the MBF of AG samples from experiment 1 and 2. It is suggested that because of sedimentation, most of the AG RBCs producing the low frequency (low frequency compared to the signal from NA RBCs) signal have already settled beyond the middle section of the vessel and below the ROI. The low SNR of 2 and features of the spectrum (all frequencies present equally) in Figure 3-26 experiment 3 NA and AG 10\% HCT indicate that in both cases RBCs had settled past the ROI. This is also reflected in the MBF plots in Figure 3-27 where the MBF values for both NA and AG samples are the lowest amongst all the 10\% HCT experimental samples measured.

With sedimentation effects explained, the increase in MBF from the middle section of the vessels with aggregation for both optical wavelengths agrees with the low frequency experimental and theoretical results; Hysi et al [2] reported increase in MBF associated with aggregation.

\subsection{Photoacoustic oxygen saturation for aggregated samples}

The SA for $850 \mathrm{~nm}$ was higher than $750 \mathrm{~nm}$ the as shown in Figure 3-14 and Figure 3-19. The sample preparation required for thorough mixing in the tube to keep it fairly homogeneous and to prevent settling. The mixing made the RBCs oxygenated. The $\mathrm{SO}_{2}$ plot in Figure 3-23 
correspondingly shows high level of oxygenation. It has been shown that RBC aggregation decreases oxygen unloading and increases $\mathrm{SO}_{2}$ [24]. A higher $\mathrm{SO}_{2}$ for $\mathrm{AG} \mathrm{RBCs}$ was also reported in the low frequency study by Hysi et al [3] as shown in Figure 1-12. The AG samples in Figure 3-23 had higher $\mathrm{SO}_{2}$ than the NA samples, which supports the higher level of oxygenation in AG RBCs reported by Tateishi [24]. Calculations were done for the 40\% HCT samples only where sedimentation was not considered to be a significant factor in the signal features. 


\section{Chapter 5 : Preliminary and Future work}

\subsection{Numerical simulations to validate experimental PA data}

Monte Carlo simulation code used to model PA signals from tissue [83], [84] were modified to simulate the illumination geometry of LZ550 transducer and insilico vessels with absorption and scattering profiles of RBC aggregates were used to generate fluence distribution and energy deposition in the blood vessel. Based on the suggested distribution in Figure 4-1 the aggregates were first uniformly distributed throughout the vessel and later were packed at the distal edge of the vessel to simulate sedimentation. Some preliminary simulation results are shown below.
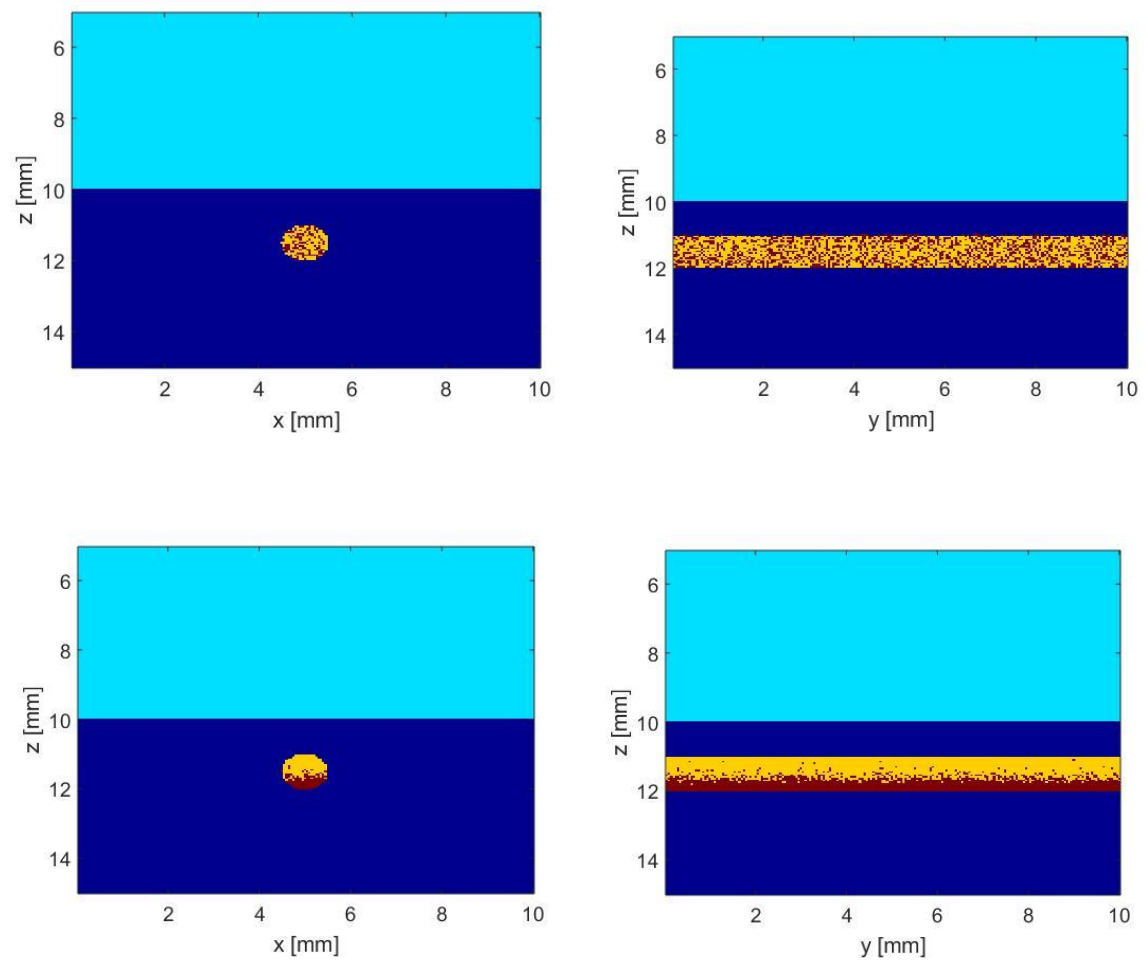

Figure 5-1: Simulated vessel geometries. (Top) 40\% HCT blood in a $1 \mathrm{~mm}$ diameter vessel with aggregates uniformly distributed. (Bottom) 40\% HCT blood with the aggregates sedimented at the distal edge. 
Figure 5-1 shows the structure for the blood vessels left images show the xz profies and the right images show the yz profiles. Optical properties were assigned to be water [92] above the phantom, an agar [93] phantom, a vessel with plasma [94] and RBC [44] aggregates. For non sedimented case a total of photons were simulated and for the vessel with sedimentation photons were simulated. Figure 5-2 shows the simulated fluence pattern, and Figure 5-3 shows the energy deposition in the vessel and phantom.
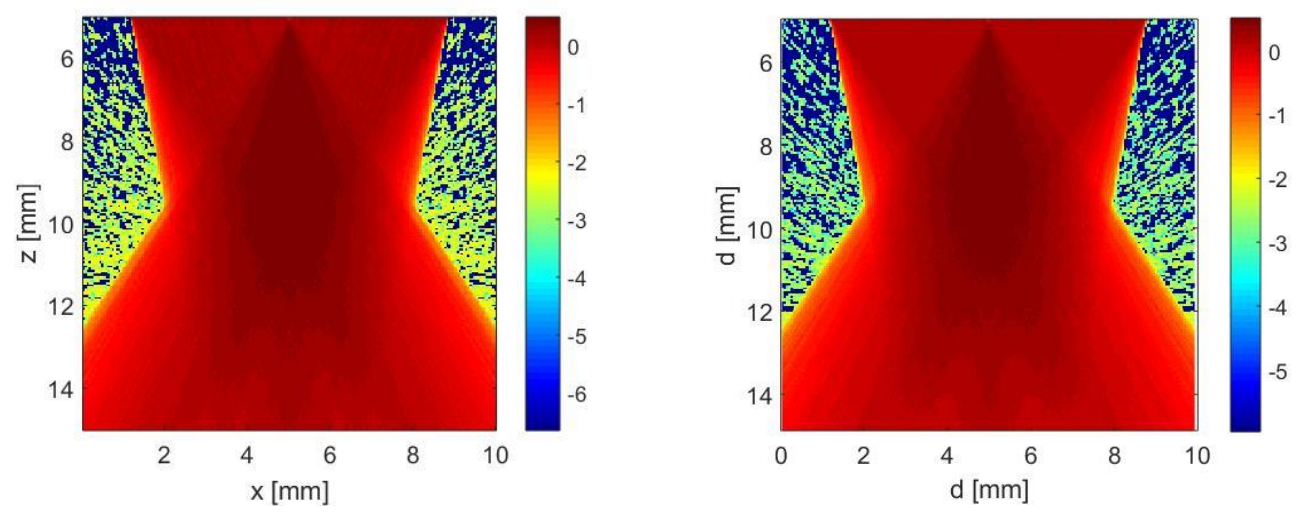

Figure 5-2: (Left) simulated fluence for sample with no sedimentation, (right) simulated fluence for sample with sedimentation.
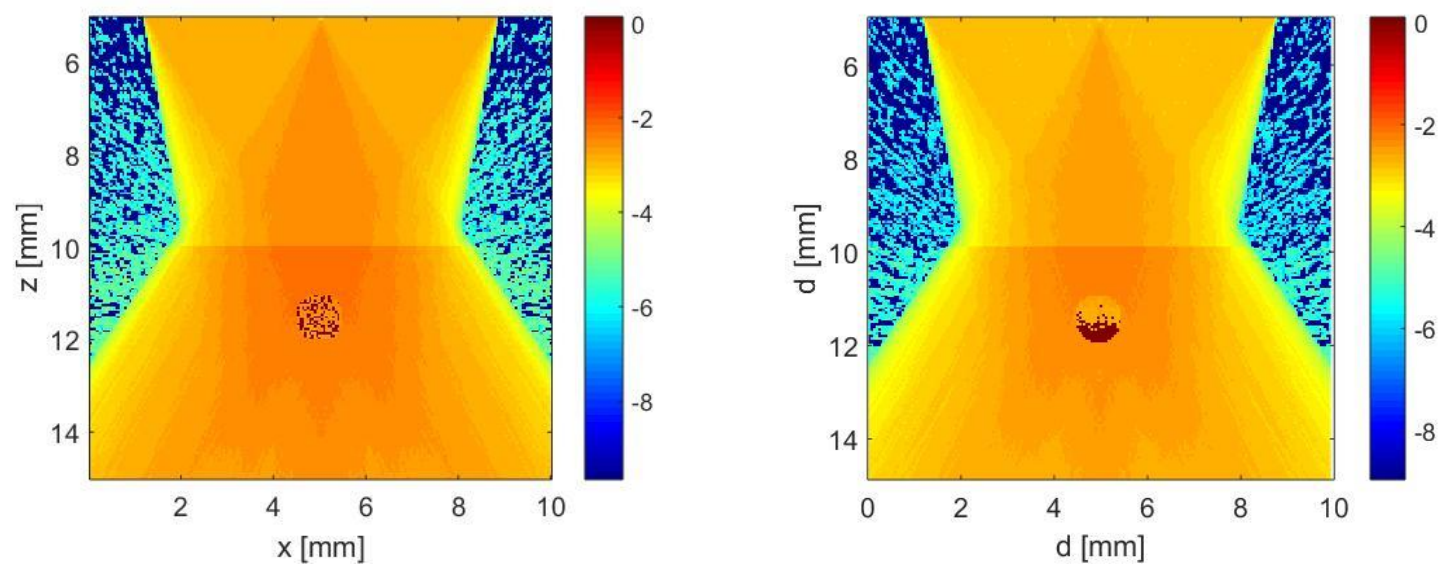

Figure 5-3: Energy deposition map for (left) sample simulated with no sedimentation and (right) sample with sedimentation. 
Figure 5-4 shows the simulated A lines from the vessels for one US detection element. The signal from the RBCs distributed uniformly starts right at the start of the proximal edge of the vessel at $11 \mathrm{~mm}$ mark and has amplitude of about $1 \mathrm{MPa}$. Whereas the signal from the vessel with blood that is sedimented starts from the middle of the vessel at about $11.6 \mathrm{~mm}$ mark. However the peak value is about $3 \mathrm{MPa}$ which is much higher than the non sedimented vessel. The signal for uniformly distributed aggregates also show more fluctuations, which may be related to the discrete presence of aggregates in the suspension. In contrast the signal from the sedimented sample appears smoother since all the aggregates are packed and form a concentrated continuous layer at the distal edge.
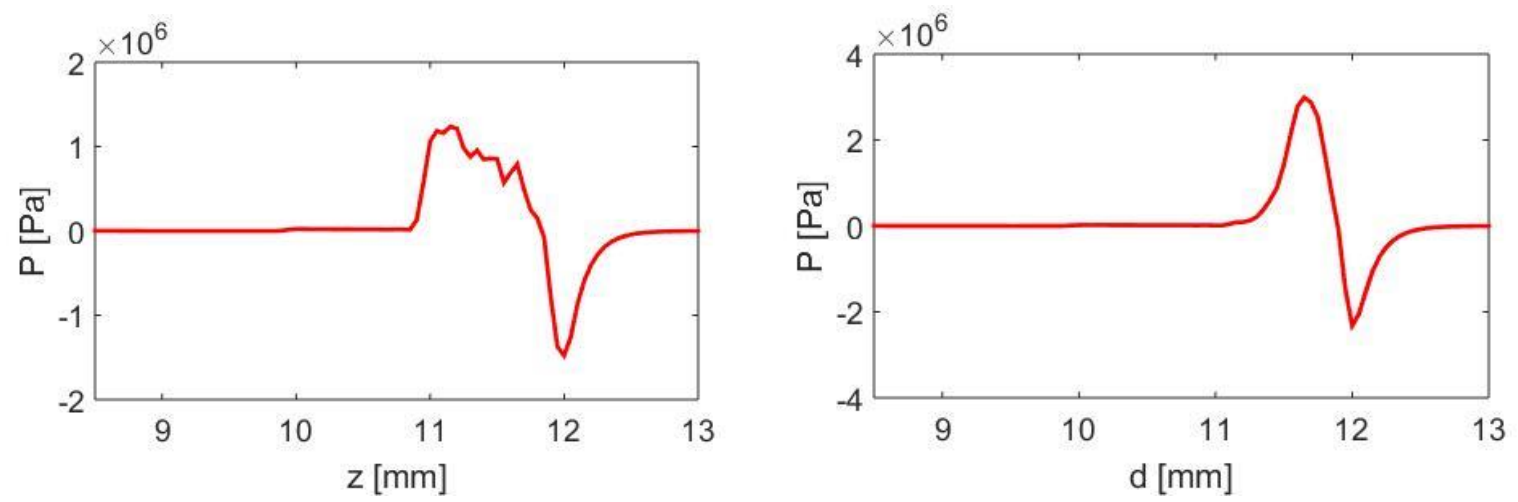

Figure 5-4: Simulated Alines for the middle element (left) uniform aggregate distribution, (right) sedimented RBC aggregates.

The preliminary results show that the SA is dependent on the energy deposition density in the tissue, which depends on the fluence and distribution of optical absorbers in a sample. Thus the discussions on the influence of sedimentation on the SA as seen in the experiments for this thesis look plausible.

The simulation can be expanded to detect PA signal using multiple elements, and conventional PA delay and sum algorithm [95] can be used to beamform the signals. Reconstructed B mode 
images from the simulated PA signals could be used to verify the experimental increase in B mode echogenicity.

\subsection{Experimental PA and US comparison}

Both PA and US data were collected in this study for all phantoms. The US data could be analyzed to find possible correlation with changes observed in PA due to aggregation. Changes in SS and MBF with aggregation in US are well studied and have been presented in the introduction section. This may provide a benchmark for comparing the only two modalities capable of investigating aggregation in vivo.

\subsection{Experimental investigation in flow}

Blood aggregation studies in stasis are the first steps in quantifying the effect of aggregation on the PA signal. However this study has limitations, effect of sedimentation changes distribution of light absorption by the RBCs. In flow the distribution of aggregates is different. The shearing velocity affects formation of aggregates, the RBCs migrate axially and HCT in microcirculation varies significantly, which can be as low as $8 \%$ in capillaries [96]. A natural progression from the current study is to study aggregation in flow conditions in vitro and in vivo. This way the effect of blood sedimentation that prevails in static studies can be circumvented.

Some preliminary work involving in vivo PA imaging of blood vessels have been conducted.

Figure 5-5 shows the US and PA images obtained from vessel of a chicken chorioallantoic membrane (CAM) model [97]. This assay allows direct access to vessels without any intervening layers of skin tissue which facilitates PA investigations. 


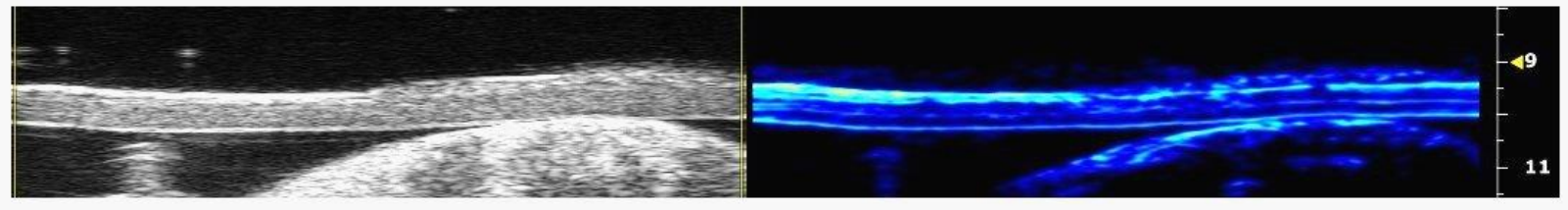

Figure 5-5: US (left) and PA (right) image from the VEVO 2100 LAZR screen showing blood flow in vivo. Obtained noninvasively from a CAM model. The PA contrast shows the phenomenon of axial migration.

It was interesting to see clear evidence of axial migration [13] in PA image as a bright streak at the middle of the vessel. This phenomenon is not evident in the US image. The advantage of absorber generated signal contrast in PA may allow for better imaging and signal acquisition to study hemodynamic relations in vivo.

For freshly drawn blood from the CAM when $3 \% \mathrm{w} / \mathrm{v}$ concentration DEX-70 was used to suspend RBCs, AG clusters were formed. In comparison RBCs suspended in PBS were NA. In vivo aggregation however was a different challenge. Using lipopolysaccharide (LPS) [4] or DEX-500 [38] infusion could produce in vivo CAM aggregation. Even though the chicken RBCs are elliptical in shape and slightly larger than human RBCs, the relative ease of using CAM model in comparison to other animal models offer substantial advantages. 


\section{Chapter 6 : Conclusion}

The findings presented in this thesis describe the first high frequency photoacoustic detection of red blood cell aggregation and oxygen saturation. Results show that the combined effect of optical fluence, local absorber concentration and distribution which govern optical energy deposition must be considered in conjunction with the size of the optical absorbers in order to interpret the impact of aggregation on the spectral parameters. Spectral slope obtained from signals with high signal to noise ratio show a decrease in slope $(-0.38 \mathrm{~dB} / \mathrm{MHz}$ and -0.63 $\mathrm{dB} / \mathrm{MHz}$ for NA and AG samples respectively) with increase in absorber size. The midband fit was however more influenced by the local energy deposition and showed a reverse trend with aggregation between different regions of the vessels. Using two illuminating wavelengths the oxygen saturation was quantified for the aggregates, which indicated a high level of oxygenation for both aggregated and non-aggregated red blood cells. The fact that during sample preparation the RBCs were exposed to oxygen in the ambient laboratory air may explain why all the samples were oxygenated. In addition the aggregated samples had slightly higher oxygen saturation level. This result supports previous publications that reported impaired release of oxygen from aggregated red blood cells. Photoacoustic blood characterization, even though in its early stages, holds a promising future in in vivo applications. 


\section{Appendix: Data from experiment 2 and 3}

\section{US and PA envelope 850 nm}
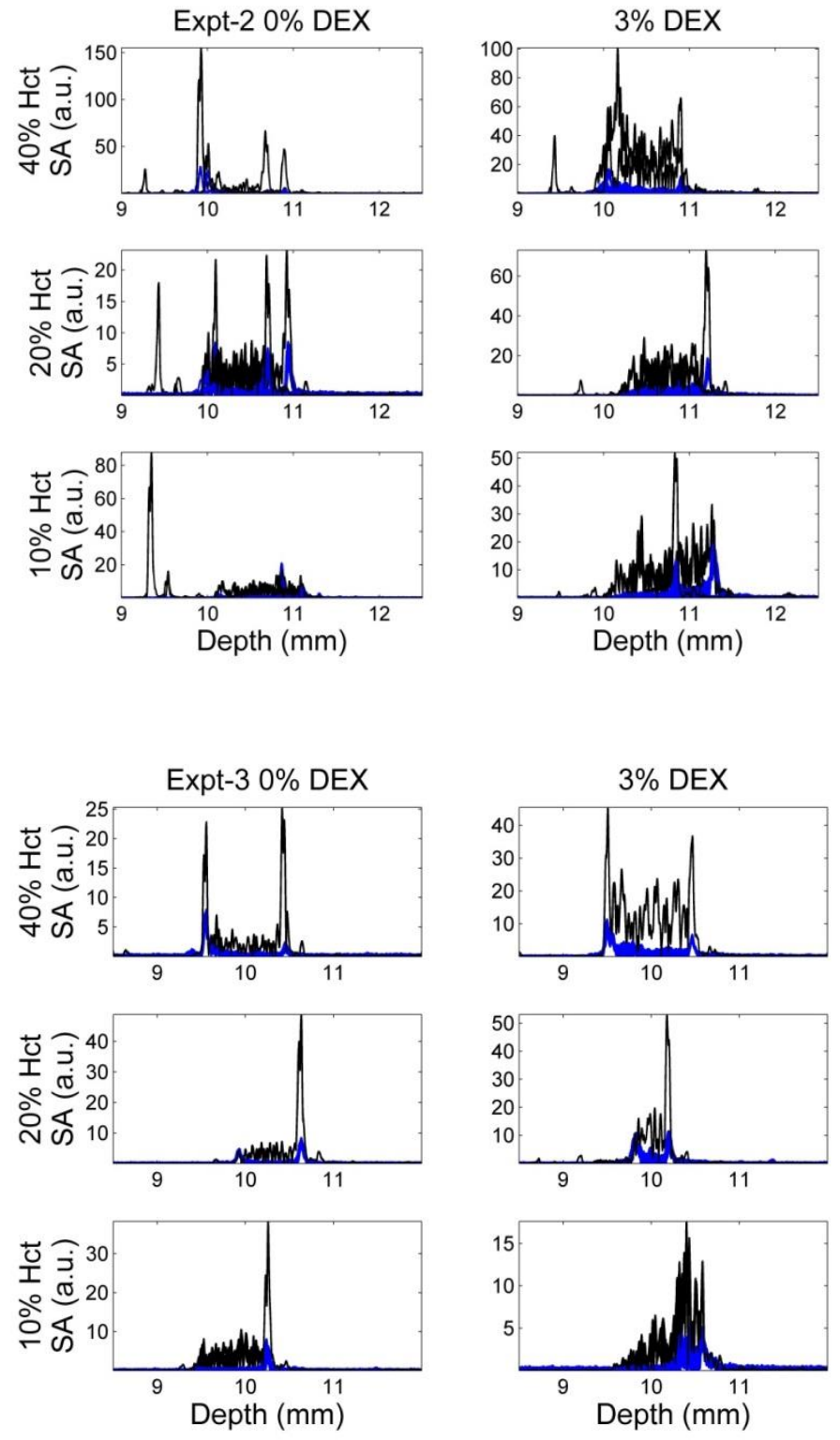

Figure A-0-1: Envelopes of US (black) and PA (blue) A lines. US A lines were used to determine the beginning, middle and end of the vessels. Data from (top) experiment 2 (bottom) experiment 3. 


\section{$B$ mode images $850 \mathrm{~nm}$}
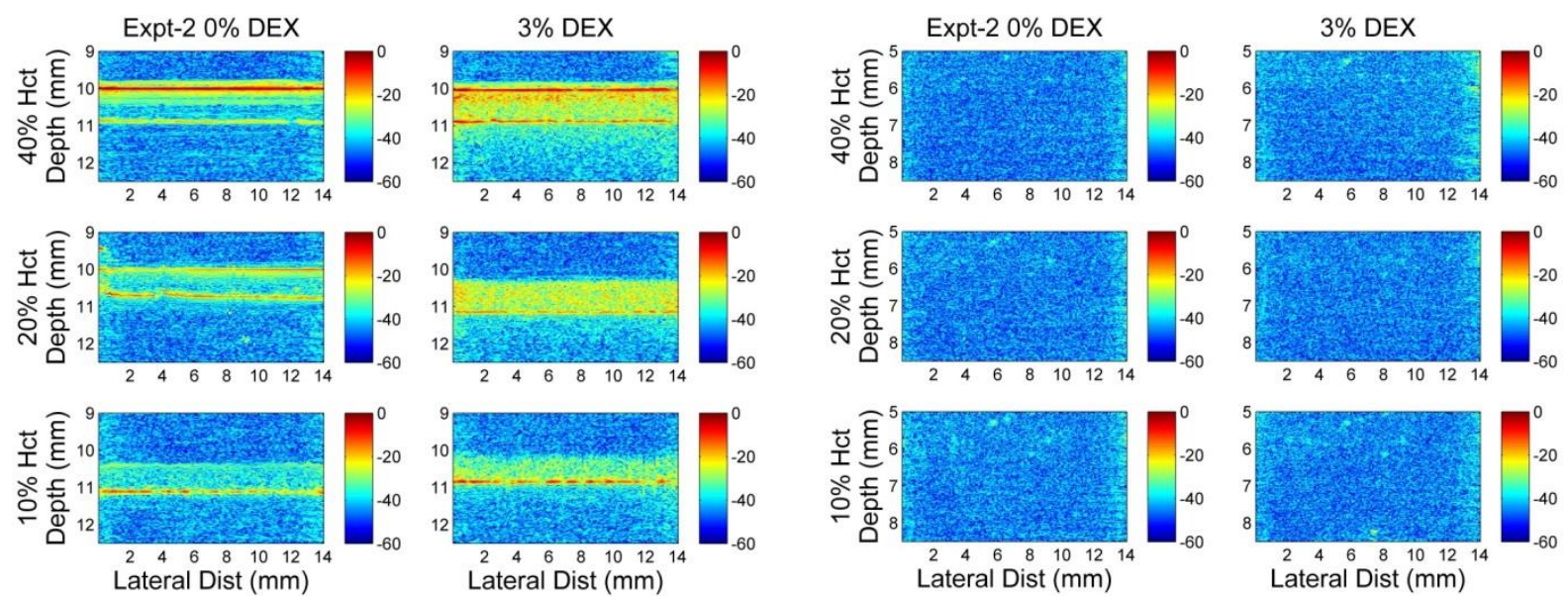

Figure A-0-2: PA ROI with vessel realigned (left) and (right) noise from a region between the transducer and phantom. Data shown from experiment 2 is shown here.
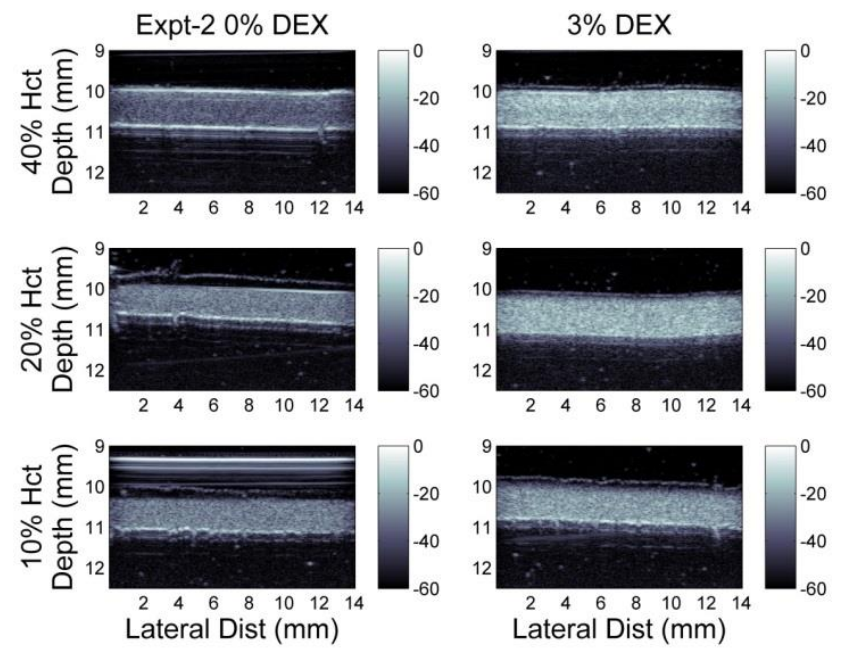

Figure A-0-3: Reconstructed US B mode image. Data from experiment 2 is shown here. 

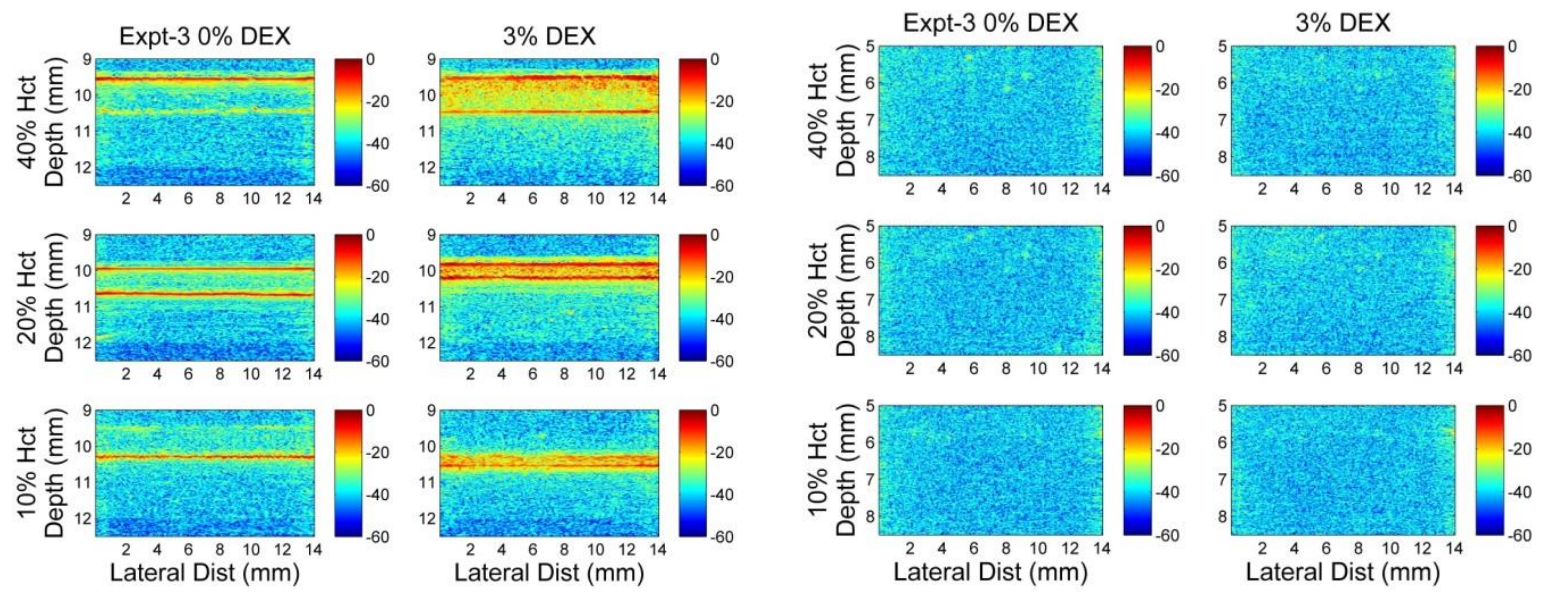

Figure A-0-4: PA ROI with vessel realigned (left) and (right) noise from a region between the transducer and phantom. Data from experiment 3 is shown here.
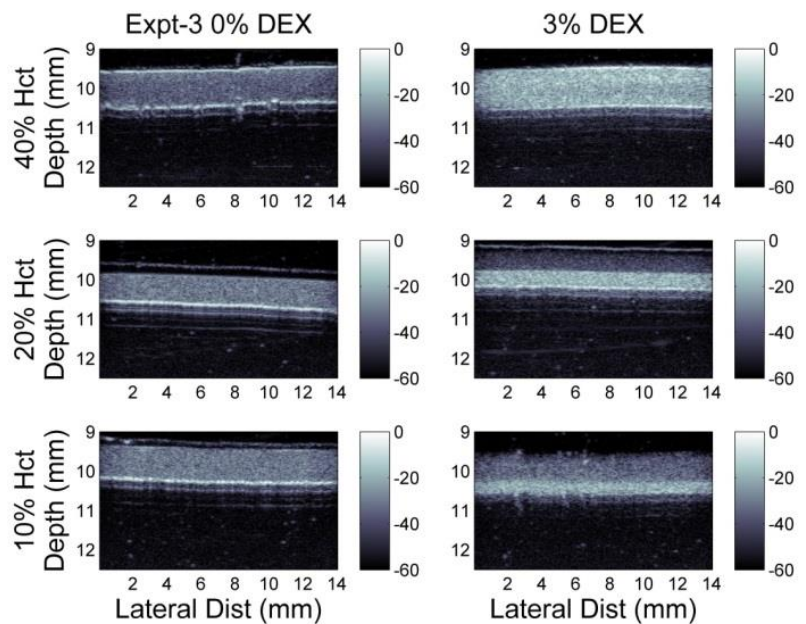

Figure A-0-5: Reconstructed US B mode image. Data from experiment 3 is shown here. 


\section{References}

[1] R. K. Saha and M. C. Kolios, "A simulation study on photoacoustic signals from red blood cells," J. Acoust. Soc. Am., vol. 129, no. 5, pp. 2935-2943, 2011.

[2] E. Hysi, R. K. Saha, and M. C. Kolios, "On the use of photoacoustics to detect red blood cell aggregation,” Biomed. Opt. Express, vol. 3, no. 9, pp. 2326-2338, 2012.

[3] E. Hysi, R. K. Saha, and M. C. Kolios, "Photoacoustic ultrasound spectroscopy for assessing red blood cell aggregation and oxygenation," J. Biomed. Opt., vol. 17, no. 12, p. 125006-1, 2012.

[4] J. Tripette, A. Y. Denault, L. Allard, B. Chayer, L. P. Perrault, and G. Cloutier, "Ultrasound Monitoring of RBC Aggregation as a Real-Time Marker of the Inflammatory Response in a Cardiopulmonary Bypass Swine Model.," Crit. Care Med., vol. 41, no. 8, pp. e171-e178, 2013.

[5] O. K. Baskurt and H. J. Meiselman, "Erythrocyte aggregation: Basic aspects and clinical importance," Clin. Hemorheol. Microcirc., vol. 53, no. 1, pp. 23-37, 2013.

[6] O. Baskurt, B. Neu, and H. J. Meiselman, Red blood cell aggregation. Boca Ralton, FL: CRC Press, 2011.

[7] E. Dzierzak and S. Philipsen, "Erythropoiesis: Development and Differentiation," Cold Spring Harb. Perspect. Med., vol. 3, no. 4, p. a011601, Apr. 2013.

[8] C. Li and L. V. Wang, "Photoacoustic tomography and sensing in biomedicine," Phys. Med. Biol., vol. 54, no. 19, pp. R59-R97, 2009.

[9] T. Ahrens, K. Rutherford, and K. A. R. Basham, Essentials of oxygenation: implication for clinical practice. Boston, MA: Jones \& Bartlett Publishing, 1993.

[10] F. B. Jensen, "The dual roles of red blood cells in tissue oxygen delivery: oxygen carriers and regulators of local blood flow," J. Exp. Biol., vol. 212, no. 21, pp. 3387-3393, 2009.

[11] N. N. Barvitenko, M. Aslam, J. Filosa, E. Matteucci, M. Nikinmaa, A. Pantaleo, C. Saldanha, and O. K. Baskurt, "Tissue Oxygen Demand in Regulation of the Behavior of the Cells in the Vasculature," Microcirculation, vol. 20, no. 6, pp. 484-501, 2013.

[12] S. Kim, P. K. Ong, O. Yalcin, M. Intaglietta, and P. C. Johnson, "The cell-free layer in microvascular blood flow," Biorheology, vol. 46, no. 3, pp. 181-189, 2009.

[13] H. L. Goldsmith, G. R. Cokelet, and P. Gaehtgens, "Robin Fahraeus: evolution of his concepts in cardiovascular physiology," Am. J. Physiol.-Heart Circ. Physiol., vol. 257, no. 3, pp. H1005-H1015, 1989.

[14] H. J. Meiselman, "Red blood cell aggregation: 45 years being curious," Biorheology, vol. 46, no. 1, pp. 1-19, 2009.

[15] S. Asakura and F. Oosawa, "Interaction between particles suspended in solutions of macromolecules," J. Polym. Sci., vol. 33, no. 126, pp. 183-192, 1958.

[16] P. Steffen, C. Verdier, and C. Wagner, "Quantification of depletion-induced adhesion of red blood cells," Phys. Rev. Lett., vol. 110, no. 1, p. 018102, 2013.

[17] E. Ponder, "On sedimentation and rouleaux formation-II," Q. J. Exp. Physiol., vol. 16, no. 2, pp. 173-194, 1926.

[18] S. Kim, J. Zhen, A. S. Popel, M. Intaglietta, and P. C. Johnson, "Contributions of collision rate and collision efficiency to erythrocyte aggregation in postcapillary venules at low flow rates," Am. J. Physiol. Circ. Physiol., vol. 293, no. 3, pp. H1947-54, 2007. 
[19] G. Barshtein, D. Wajnblum, and S. Yedgar, "Kinetics of linear rouleaux formation studied by visual monitoring of red cell dynamic organization," Biophys. J., vol. 78, no. 5, pp. 2470-2474, 2000.

[20] A. SzoĹ and M. Bosek, "Kinetics of red blood cell rouleaux formation studied by light scattering," J. Biomed. Opt., vol. 20, no. 2, pp. 025001-025001, 2015.

[21] T. François and G. Cloutier, "Experimental ultrasound characterization of red blood cell aggregation using the structure factor size estimator," J. Acoust. Soc. Am., vol. 122, no. 1, pp. 645-656, 2007.

[22] T. L. Fabry, "Mechanism of erythrocyte aggregation and sedimentation," Blood, vol. 70, no. 5, pp. 1572-1576, 1987.

[23] T. Kirschkamp, H. Schmid-Schönbein, A. Weinberger, and R. Smeets, "Effects of fibrinogen and a2-macroglobulin and their apheretic elimination on general blood rheology and rheological characteristics of red blood cell aggregates," Ther. Apher. Dial., vol. 12, no. 5, pp. 360-367, 2008.

[24] N. Tateishi, Y. Suzuki, I. Cicha, and N. Maeda, "O2 release from erythrocytes flowing in a narrow O2-permeable tube: Effects of erythrocyte aggregation," Am. J. Physiol. - Heart Circ. Physiol., vol. 281, no. 1, pp. H448-H456, 2001.

[25] E. Franceschini, T. H. François, F. Destrempes, and G. Cloutier, "Ultrasound characterization of red blood cell aggregation with intervening attenuating tissuemimicking phantoms," J. Acoust. Soc. Am., vol. 127, no. 2, pp. 1104-1115, 2010.

[26] B. Almog, R. Gamzu, R. Almog, J. B. Lessing, I. Shapira, S. Berliner, D. Pauzner, S. Maslovitz, and I. Levin, "Enhanced erythrocyte aggregation in clinically diagnosed pelvic inflammatory disease,” Sex. Transm. Dis., vol. 32, no. 8, pp. 484-486, 2005.

[27] L. Zilberman, O. Rogowski, M. Rozenblat, I. Shapira, J. Serov, P. Halpern, I. Dotan, N. Arber, and S. Berliner, "Inflammation-related erythrocyte aggregation in patients with inflammatory bowel disease," Dig. Dis. Sci., vol. 50, no. 4, pp. 677-683, 2005.

[28] R. B. Ami, G. Barshtein, D. Zeltser, Y. Goldberg, I. Shapira, A. Roth, G. Keren, H. Miller, V. Prochorov, A. Eldor, and others, "Parameters of red blood cell aggregation as correlates of the inflammatory state," Am. J. Physiol.-Heart Circ. Physiol., vol. 280, no. 5, pp. H1982-H1988, 2001.

[29] J.-J. Monsuez, J. Dufaux, D. Vittecoq, P. Flaud, and E. Vicaut, "Hemorheology in asymptomatic HIV-infected patients.," Clin. Hemorheol. Microcirc., vol. 23, no. 1, pp. 5966, 1999.

[30] R. R. Puniyani, V. S. Agashe, V. ARNAPURNA, and S. R. Daga, "Haemorheological profile in cases of chronic infections," Clin. Hemorheol., vol. 8, no. 5, pp. 595-602, 1988.

[31] H. J. Meiselman, "Hemorheologic alterations in hypertension: Chicken or egg?," Clin. Hemorheol. Microcirc., vol. 21, no. 3-4, pp. 195-200, 1999.

[32] S. Chien and K.-M. Jan, "Ultrastructural basis of the mechanism of rouleaux formation," Microvasc. Res., vol. 5, no. 2, pp. 155-166, 1973.

[33] S. Chen, G. Barshtein, B. Gavish, Y. Mahler, and S. Yedgar, "Monitoring of red blood cell aggregability in a flow-chamber by computerized image analysis," Clin. Hemorheol., vol. 14, no. 4, pp. 497-508, 1994.

[34] S. Chen, B. Gavish, S. Zhang, Y. Mahler, and S. Yedgar, "Monitoring of erythrocyte aggregate morphology under flow by computerized image analysis," Biorheology, vol. 32, no. 4, pp. 487-496, 1995. 
[35] E. Vicaut, X. Hou, L. Decuypere, A. Taccoen, and M. Duvelleroy, "Red blood cell aggregation and microcirculation in rat cremaster muscle," Int. J. Microcirc., vol. 14, no. 12, pp. 14-21, 1994.

[36] M. J. Pearson and H. H. Lipowsky, "Influence of erythrocyte aggregation on leukocyte margination in postcapillary venules of rat mesentery," Am. J. Physiol. Circ. Physiol., vol. 279, no. 4, pp. H1460-H1471, 2000.

[37] W. G. Zijlstra, "Syllectometry, a new method for studying rouleaux formation of red blood cells," Acta Phys. Pharm. Neerl. vol. 7, pp. 153-154, 1958.

[38] S. Kim, A. S. Popel, M. Intaglietta, and P. C. Johnson, "Aggregate formation of erythrocytes in postcapillary venules," Am. J. Physiol. - Heart Circ. Physiol., vol. 288, no. 2, pp. H584-H590, 2005.

[39] X. Xu, L. Yu, and Z. Chen, "Optical clearing of flowing blood using dextrans with spectral domain optical coherence tomography," J. Biomed. Opt., vol. 13, no. 2, p. 021107-1, 2008.

[40] M. Kinnunen, "A pulsed photoacoustic technique for studying red blood cell sedimentation," J. Biomed. Photonics Eng., vol. 1, no. 1, pp. 81-89, 2015.

[41] L. Duyens, "The flattering of the absorption spectrum of suspensions, as compared to that of solutions," Biochim. Biophys. Acta, vol. 19, pp. 1-12, 1956.

[42] D. Yim, G. V. Baranoski, B. W. Kimmel, T. F. Chen, and E. Miranda, "A Cell-Based Light Interaction Model for Human Blood," in Computer Graphics Forum, 2012, vol. 31, no. 2, pp. 845-854.

[43] E. I. Galanzha and V. P. Zharov, "In vivo photoacoustic and photothermal cytometry for monitoring multiple blood rheology parameters," Cytometry A, vol. 79A, no. 10, pp. 746757, 2011.

[44] N. Bosschaart, G. J. Edelman, M. C. Aalders, T. G. van Leeuwen, and D. J. Faber, "A literature review and novel theoretical approach on the optical properties of whole blood," Lasers Med. Sci., vol. 29, no. 2, pp. 453-479, 2014.

[45] V. V. Tuchin, X. Xu, and R. K. Wang, "Dynamic optical coherence tomography in studies of optical clearing, sedimentation, and aggregation of immersed blood," Appl. Opt., vol. 41, no. 1, pp. 258-271, 2002.

[46] J. Strzelecka, M. Bosek, and B. Grzegorzewski, "Optical properties of deposit of red blood cells suspended in dextran solutions," Opt. Appl., vol. 40, no. 1, pp. 177-185, 2010.

[47] A. Merino, P. Hauptman, L. Badimon, J. J. Badimon, M. Cohen, V. Fuster, and M. Goldman, "Echocardiographic 'smoke' is produced by an interaction of erythrocytes and plasma proteins modulated by shear forces," J. Am. Coll. Cardiol., vol. 20, no. 7, pp. 1661$1668,1992$.

[48] D.-G. Paeng, R. Y. Chiao, and K. K. Shung, "Echogenicity variations from porcine blood II: the 'bright ring' under oscillatory flow," Ultrasound Med. Biol., vol. 30, no. 6, pp. 815825, Jun. 2004.

[49] F. L. Lizzi, E. J. Feleppa, S. K. Alam, and C. X. Deng, "Ultrasonic spectrum analysis for tissue evaluation," Pattern Recognit. Lett., vol. 24, no. 4, pp. 637-658, 2003.

[50] G. Cloutier and Z. Qin, "Ultrasound backscattering from non-aggregating and aggregating erythrocytes-A review," Biorheology, vol. 34, no. 6, pp. 443-470, 1997.

[51] F. L. Lizzi, M. Greenebaum, E. J. Feleppa, M. Elbaum, and D. J. Coleman, "Theoretical framework for spectrum analysis in ultrasonic tissue characterization," J. Acoust. Soc. Am., vol. 73, no. 4, pp. 1366-1373, 1983. 
[52] K. K. Shung, Y. W. Yuan, D. Y. Fei, and J. M. Tarbell, "Effect of flow disturbance on ultrasonic backscatter from blood," J. Acoust. Soc. Am., vol. 75, no. 4, pp. 1265-1272, 1984.

[53] J. Tripette, L.-C. Nguyen, L. Allard, P. Robillard, G. Soulez, and G. Cloutier, "In vivo venous assessment of red blood cell aggregate sizes in diabetic patients with a quantitative cellular ultrasound imaging method: Proof of concept," PLoS One, vol. 10, no. 4, 2015. doi:10.1371/journal.pone.0124712.

[54] J. R. Thiele, K. Goerendt, G. Bjoern Stark, and S. U. Eisenhardt, "Real-time digital imaging of leukocyte-endothelial interaction in ischemia-reperfusion injury (IRI) of the rat cremaster muscle," J. Vis. Exp., no. 66, 2012. doi:10.3791/3973.

[55] L. S. Ritter, J. A. Orozco, B. M. Coull, and P. F. McDonagh, "Leukocyte accumulation and hemodynamic changes in the cerebral microcirculation during early reperfusion after stroke," Stroke, vol. 31, no. 5, pp. 1153-1161, 2000.

[56] L. S. Ritter, D. S. Wilson, S. K. Williams, J. G. Copeland, and P. F. McDonagh, "Early in reperfusion following myocardial ischemia, leukocyte activation is necessary for venular adhesion but not capillary retention," Microcirculation, vol. 2, no. 4, pp. 315-327, 1995.

[57] J. Y. Hokama, L. S. Ritter, G. Davis-Gorman, A. D. Cimetta, J. G. Copeland, and P. F. McDonagh, "Diabetes enhances leukocyte accumulation in the coronary microcirculation early in reperfusion following ischemia," J. Diabetes Complications, vol. 14, no. 2, pp. 96$107,2000$.

[58] L. V. Wang and S. Hu, "Photoacoustic tomography: In vivo imaging from organelles to organs," Science, vol. 335, no. 6075, pp. 1458-1462, 2012.

[59] R. S. Cobbold, Foundations of biomedical ultrasound. New York , NY: Oxford University Press, 2006.

[60] S. Zackrisson, S. M. W. Y. Van De Ven, and S. S. Gambhir, "Light in and sound out: Emerging translational strategies for photoacoustic imaging," Cancer Res., vol. 74, no. 4, pp. 979-1004, 2014.

[61] K. Briggs, A. Al Mahrouki, J. Nofiele, A. El-Falou, M. Stanisz, H. C. Kim, M. C. Kolios, and G. J. Czarnota, "Non-invasive monitoring of ultrasound-stimulated Microbubble radiation enhancement using photoacoustic imaging," Technol. Cancer Res. Treat., vol. 13, no. 5, pp. 435-444, 2014.

[62] M. P. Patterson, C. B. Riley, M. C. Kolios, and W. M. Whelan, "Optoacoustic characterization of prostate cancer in an in vivo transgenic murine model," J. Biomed. Opt., vol. 19, no. 5, pp. pp. 056008-056008, 2014.

[63] M. A. Juratli, M. Sarimollaoglu, D. A. Nedosekin, A. V. Melerzanov, V. P. Zharov, and E. I. Galanzha, "Dynamic fluctuation of circulating tumor cells during cancer progression," Cancers, vol. 6, no. 1, pp. 128-142, 2014.

[64] M. A. Juratli, M. Sarimollaoglu, E. R. Siegel, D. A. Nedosekin, E. I. Galanzha, J. Y. Suen, and V. P. Zharov, "Real-time monitoring of circulating tumor cell release during tumor manipulation using in vivo photoacoustic and fluorescent flow cytometry," Head Neck, vol. 36, no. 8, pp. 1207-1215, 2014.

[65] H. Ke, S. Tai, and L. V. Wang, "Photoacoustic thermography of tissue," J. Biomed. Opt., vol. 19, no. 2, pp. pp.026003-026003, 2014.

[66] M. Alhamami, M. C. Kolios, and J. Tavakkoli, "Photoacoustic detection and optical spectroscopy of high-intensity focused ultrasound-induced thermal lesions in biologic tissue," Med. Phys., vol. 41, no. 5, p. 053502, 2014. 
[67] B. Wang, A. Karpiouk, D. Yeager, J. Amirian, S. Litovsky, R. Smalling, and S. Emelianov, "Intravascular photoacoustic imaging of lipid in atherosclerotic plaques in the presence of luminal blood," Opt. Lett., vol. 37, no. 7, pp. 1244-1246, 2012.

[68] B. Lashkari and A. Mandelis, "Coregistered photoacoustic and ultrasonic signatures of early bone density variations," J. Biomed. Opt., vol. 19, no. 3, pp.036015-036015, 2014.

[69] S. Hu and L. V. Wang, "Optical-resolution photoacoustic microscopy: Auscultation of biological systems at the cellular level," Biophys. J., vol. 105, no. 4, pp. 841-847, 2013.

[70] A. G. Bell, "On the production and reproduction of sound by light," Am. J. Sci., no. 118, pp. 305-324, 1880.

[71] P. Beard, "Biomedical photoacoustic imaging," Interface Focus, vol. 1, no. 4, pp. 602-631, 2011.

[72] G. J. Diebold and P. J. Westervelt, "The photoacoustic effect generated by a spherical droplet in a fluid," J. Acoust. Soc. Am., vol. 84, no. 6, pp. 2245-2251, 1988.

[73] G. Xu, I. A. Dar, C. Tao, X. Liu, C. X. Deng, and X. Wang, "Photoacoustic spectrum analysis for microstructure characterization in biological tissue: A feasibility study," Appl. Phys. Lett., vol. 101, no. 22, p. 221102, 2012.

[74] S. Wang, C. Tao, X. Wang, and X. Liu, "Quantitative detection of stochastic microstructure in turbid media by photoacoustic spectral matching," Appl. Phys. Lett., vol. 102, no. 11, p. $114102,2013$.

[75] E. M. Strohm, I. Gorelikov, N. Matsuura, and M. C. Kolios, "Acoustic and photoacoustic characterization of micron-sized perfluorocarbon emulsions," J. Biomed. Opt., vol. 17, no. 9, pp. 0960161-0960169, 2012.

[76] R. K. Saha and M. C. Kolios, "Effects of erythrocyte oxygenation on optoacoustic signals," J. Biomed. Opt., vol. 16, no. 11, pp. 115003-1150039, 2011.

[77] R. K. Saha, "A simulation study on the quantitative assessment of tissue microstructure with photoacoustics," IEEE Trans. Ultrason. Ferroelectr. Freq. Control, vol. 62, no. 5, pp. 881-895, 2015.

[78] Z. Guo, L. Li, and L. V. Wang, "On the speckle-free nature of photoacoustic tomography," Med. Phys., vol. 36, no. 9, pp. 4084-4088, 2009.

[79] R. E. Kumon, C. X. Deng, and X. Wang, "Frequency-domain analysis of photoacoustic imaging data from prostate adenocarcinoma tumors in a murine model," Ultrasound Med. Biol., vol. 37, no. 5, pp. 834-839, 2011.

[80] X. Wang, X. Xie, G. Ku, L. V. Wang, and G. Stoica, "Noninvasive imaging of hemoglobin concentration and oxygenation in the rat brain using high-resolution photoacoustic tomography," J. Biomed. Opt., vol. 11, no. 2, pp. 024015-024015-9, 2006.

[81] F. B. Jensen, "Red blood cell $\mathrm{pH}$, the Bohr effect, and other oxygenation-linked phenomena in blood O2 and CO2 transport," Acta Physiol. Scand., vol. 182, no. 3, pp. 215-227, 2004.

[82] L. H. Wang and S. L. Jacques, "Monte Carlo modeling of light transport in multi-layered tissues in standard C," 1992, Laser Biology Res. Lab., Univ. of Texas M.D. Anderson Cancer Center.

[83] S. L. Jacques and G. Paltauf, "Modeling pressure waves generated by pulsed laser irradiation of irregularly shaped absorbing objects within media," in BiOS 2001 The International Symposium on Biomedical Optics, 2001, pp. 90-100.

[84] A. Zam, S. L. Jacques, S. Alexandrov, Y. Li, and M. J. Leahy, "Modelling cerebral blood oxygenation using Monte Carlo XYZ-PA,” in SPIE BiOS, 2013, pp. 858013-858013. 
[85] O. K. Baskurt, Handbook of hemorheology and hemodynamics, vol. 69. Amsterdam, Netherlands: IOS press, 2007.

[86] J. Schindelin, I. Arganda-Carreras, E. Frise, V. Kaynig, M. Longair, T. Pietzsch, S. Preibisch, C. Rueden, S. Saalfeld, B. Schmid, J.-Y. Tinevez, D. J. White, V. Hartenstein, K. Eliceiri, P. Tomancak, and A. Cardona, "Fiji: an open-source platform for biological-image analysis," Nat. Methods, vol. 9, no. 7, pp. 676-682, 2012.

[87] "A Threshold Selection Method from Gray-Level Histograms," IEEE Trans. Syst. Man Cybern., vol. 9, no. 1, pp. 62-66, Jan. 1979.

[88] L. Vincent and P. Soille, "Watersheds in digital spaces: an efficient algorithm based on immersion simulations," IEEE Trans. Pattern Anal. Mach. Intell., vol. 13, no. 6, pp. 583598, Jun. 1991.

[89] J. E. Powers, "Ultrasound Phased Array Delay Lines Based on Quadrature Sampling Techniques," IEEE Trans. Sonics Ultrason., vol. 27, no. 6, pp. 287-294, Nov. 1980.

[90] M. Brezinski, K. Saunders, C. Jesser, X. Li, and J. Fujimoto, "Index matching to improve optical coherence tomography imaging through blood," Circulation, vol. 103, no. 15, pp. 1999-2003, 2001.

[91] G. J. Diebold, "Photoacoustic monopole radiation: waves from objects with symmetry in one, two and three dimensions,"in Photoacoust. Imaging Spectrosc., vol. 144, L. V. Wang, Ed, Boca Raton, FL: CRC Press, 2009, pp. 3-17.

[92] S. L. Jacques, “Optical properties of biological tissues: a review," Phys. Med. Biol., vol. 58, no. 11, p. R37, 2013.

[93] H. Vermeulen, "Investigation of the influence of the scattering coefficient on acousto-optic modulation efficiency in turbid phantoms," 2012.

[94] M. Meinke, G. Müller, J. Helfmann, and M. Friebel, "Optical properties of platelets and blood plasma and their influence on the optical behavior of whole blood in the visible to near infrared wavelength range," J. Biomed. Opt., vol. 12, no. 1, pp. 014024-014024, 2007.

[95] M. N. Fadhel and M. C. Kolios, "Photoacoustic Speckle and Spectral analysis of Vasculature Trees," in World Congress on Medical Physics and Biomedical Engineering, June 7-12, 2015, Toronto, Canada, 2015, pp. 1084-1087.

[96] H. H. Lipowsky, S. Usami, and S. Chien, "In vivo measurements of 'apparent viscosity" and microvessel hematocrit in the mesentery of the cat," Microvasc. Res., vol. 19, no. 3, pp. 297-319, 1980.

[97] T. Schomann, F. Qunneis, D. Widera, C. Kaltschmidt, and B. Kaltschmidt, "Improved Method for Ex Ovo-Cultivation of Developing Chicken Embryos for Human Stem Cell Xenografts," Stem Cells Int., vol. 2013, 2013. doi:10.1155/2013/960958. 